\title{
Abstracts
}

Respiration 2018;95:478-516

\section{SGP Congress 2018}

St. Gallen, Switzerland, 24-25 May, 2018 


\section{Abstracts}

Respiration 2018;95:478-516

Published online: May 9, 2018

DOI: $10.1159 / 000488898$

Abstracts are available online only, free of charge, at

www.karger.com/doi/10.1159/000488898

\section{SGP Congress 2018}

\section{St. Gallen, Switzerland, 24-25 May 2018}

\section{Poster Walk 1: Epidemiology / Health Policy}

\begin{abstract}
P01
Variability of Tidal Breathing Parameters in Preterm Infants and Associations with Respiratory Morbidity during Infancy: A Cohort-Study

J. Usemann ${ }^{1,2}$, A. Suter ${ }^{1}$, E. Zannin ${ }^{1,3}$, E. Proietti ${ }^{2,4}$, S. Fouzas ${ }^{5}$, S. Schulzke ${ }^{1}$, P. Latzin ${ }^{2}$, U. Frey ${ }^{1}$, BILD Study Group

${ }^{1}$ Pediatric Respiratory Medicine, University of Basel, Basel, ${ }^{2}$ Pediatric Respiratory Medicine, Bern University Hospital, Bern, Switzerland, ${ }^{3}$ Dipartimento di Elettronica, Milano University, Milan, Italy, ${ }^{4}$ Pediatric Respiratory Medicine, University Hospital Zurich, Zurich, Switzerland, 5 University Hospital Patras, Patras,

Greece
\end{abstract}

Objectives: As previously shown, low variability of tidal breathing and capnography parameters in preterm infants are an expression of impaired lung function and gas exchange. We tested, whether these lung parameters assessed near term are associated with respiratory morbidity during the first year of life.

Methods: Using logistic regression analysis, we investigated in a birth-cohort of 133 preterm infants, whether unsedated lung function measurements at 44 weeks post-menstrual age (variability of tidal breathing and capnographic parameters) are associated with subsequent rehospitalization, wheeze, inhalation, and home oxygen therapy in infancy. Using receiver operating characteristic (ROC) analysis, we determined whether the predictive power of BPD classification is enhanced by adding these lung function or capnographic parameters.
Results: The coefficient of variation $(\mathrm{CV})$ of $\mathrm{V}_{\mathrm{T}}\left(\mathrm{CV}_{\mathrm{VT}}\right)$ (range $4 \%-35 \%)$ and the $\mathrm{CV}$ of expired $\mathrm{CO}_{2}$ volume $\left(\mathrm{CV}_{\mathrm{VE}, \mathrm{CO} 2}\right)$ (range $5 \%-40 \%)$ were significantly lower in preterm infants with rehospitalization during infancy, compared to those without. Per interquartile range decrease in $\mathrm{CV}_{\mathrm{VT}}$ and $\mathrm{CV}_{\mathrm{VE}, \mathrm{CO} 2}$, the adjusted odds ratio $(95 \% \mathrm{CI})$ for rehospitalization increased by $2.25(1.21-4.20)$ and 2.31 (1.20-4.45), respectively. Low variability of both parameters predicted rehospitalization significantly better than BPD classification alone. The area under the ROC curve increased from 0.56 to 0.66 for both, $\mathrm{CV}_{\mathrm{VT}}$ and $\mathrm{CV}_{\mathrm{VE}, \mathrm{CO} 2}$. There was no association with the other outcomes.

Conclusions: In comparison to BPD classification alone, including variability of postnatal tidal breathing parameters increases the prediction for rehospitalization in infancy. These findings may have a potential impact on discharge procedures and monitoring strategies in preterm infants.

\section{KARGER}

E-Mail karger@karger.com www.karger.com/res
C 2018 Swiss Respiratory Society SGP.

Published by S. Karger AG, Basel.

All rights reserved. 


\section{P02 \\ Evolution of Primary Ciliary Dyskinesia (PCD) Diagnostic Testing in Europe}

F. Halbeisen ${ }^{1}$, M. Goutaki ${ }^{1}$, E. Maurer ${ }^{1}$, M. Boon ${ }^{2}$, C. Casaulta ${ }^{3}$, S. Crowley ${ }^{4}$, C. Hogg 5 , B. Karadag ${ }^{6}$, C. Koerner-Rettberg ${ }^{7}$, H. Mazurek ${ }^{8}$, U. Özçelik ${ }^{9}$, F. Santamaria ${ }^{10}$, N. Schwerk ${ }^{11}$, P. Yiallouros ${ }^{12}$, J. Lucas ${ }^{13}$, C. Kuehni ${ }^{1}$

${ }^{1}$ Institute of Social and Preventive Medicine, University of Bern, Bern, Switzerland, ${ }^{2}$ Department of Paediatrics, University Hospital Gasthuisberg, Leuven, Belgium, ${ }^{3}$ Paediatric Respiratory Medicine, Children's University Hospital of Bern, University of Bern, Bern, Switzerland, 'Unit for Paediatric Heart, Lung, Allergic Diseases, Rikshospitalet, Oslo, Norway, ${ }^{5}$ Department of Paediatrics, Primary Ciliary Dyskinesia Centre, Royal Brompton and Harefield Foundation Trust, London, United Kingdom, ${ }^{6}$ Department of Pediatric Pulmonology, Marmara University, School of Medicine, Istanbul, Turkey, ${ }^{7}$ Department of Paediatric Pneumology, University Children's Hospital of Ruhr University Bochum, Bochum, Germany, ${ }^{8}$ Department of Pneumonology and Cystic Fibrosis, Institute of Tuberculosis and Lung Disorders, Rabka, Poland, ${ }^{9}$ Department of Pediatric Pulmonology, Hacettepe University Faculty of Medicine, Ankara, Turkey, ${ }^{10}$ Department of Translational Medical Sciences, Federico II University, Naploi, Italy, ${ }^{11}$ Clinic for Paediatric Pulmonology, Allergology, Lung Transplantation, Hannover Medical School, Hannover, Germany, ${ }^{12}$ Medical School, University of Cyprus, Nicosia, Cyprus, ${ }^{13}$ Primary Ciliary Dyskinesia Centre, NIHR Biomedical Research Centre, University of Southampton, Southampton, United Kingdom

Background: Diagnosis of PCD remains complex and varies across Europe. In 2009 a consensus statement from the European Respiratory Society Taskforce on PCD recommended the use of nasal nitric oxide (nNO), electron microscopy (EM) and video microscopy (VM) as cornerstones of PCD diagnosis. This study uses individual-patient-data to describe PCD diagnostics in Europe before and after the publication of the consensus statement.

Methods: We included 12 European datasets from the international PCD (iPCD) cohort, with almost complete diagnostic data. We assessed the proportion of patients, who had been tested with $\mathrm{nNO}, \mathrm{EM}$ and VM, comparing the period before and after 2009.

Results: From the 835 included patients, 565 (67\%, 95\% CI: $60-70 \%)$ had nNO, $616(73 \%, 71-77 \%)$ had EM and $582(69 \%$, 66-73\%) had VM testing; 209 (25\%, 22-28\%) had genetic analysis. Combination of nNO, EM and VM was used in 323 patients $(39 \%$, $35-42 \%$ ). The use of EM decreased (from $88 \%$ to $64 \%$; $p<0.001$ ) after the 2009 consensus, while use of VM increased (from $66 \%$ to $74 \% ; \mathrm{p}<0.001)$. We found no time trend in nNO testing $(\mathrm{p}=0.2)$. The use of the test combination $\mathrm{nNO}, \mathrm{EM}$ and VM decreased (from $44 \%$ to $35 \% ; \mathrm{p}=0.002$ ). There was significant heterogeneity between countries.

Conclusions: Overall, we found poor adherence to the 2009 consensus recommendations, due to the decrease in use of EM and the low use of test combinations. This may result in both underand over-diagnosis of PCD. To improve PCD diagnosis, we must implement the evidence-based guidelines published by the 2016 ERS PCD Taskforce.

Funding: FP7 grant 305404, SNF 32003B-162820, Milena Carvajal -Pro Kartagener, Lung Leagues Bern, Ticino and Vaud, COST BM1407.

\section{P03 \\ Cost-Effectiveness of School-Based Tuberculosis Screening for Migrant Children Arriving in Low- Incidence Countries - Evidence from Switzerland}

\author{
J. Usemann ${ }^{1}$, M. Ledergerber ${ }^{2}$, G. Fink ${ }^{3}$, N. Ritz ${ }^{4,5}$ \\ ${ }^{1}$ Pediatric Respiratory Medicine, University of Basel, \\ ${ }^{2}$ Medizinische Dienste, Kinder- und Jugendgesundheitsdienst, \\ ${ }^{3}$ Swiss Tropical and Public Health Institute, ${ }^{4}$ Pediatric Infectious \\ Disease and Vaccinology, University of Basel, Basel, Switzerland, \\ ${ }^{5}$ Department of Pediatrics, Royal Children's Hospital Melbourne, \\ Melbourne, VIC, Australia
}

Background: Tuberculosis (TB) screening programs in migrant children have become important, as the WHO recommends diagnosis and treatment of latent tuberculosis infection (LTBI), particularly in young children. This study evaluated the cost-effectiveness of TB screening for migrant children arriving in a lowincidence country.

Methods: Retrospective analysis of a school-based TB screening program in Switzerland. Migrant children aged 5-18 years were screened using a tuberculin skin test (TST). Cost for TB screening and treatment were calculated from records of the University Children's Hospital Basel. Cost impact was analyzed as the difference between cost of treatment for active TB and screening plus treatment of LTBI. Cost per disability-adjusted life years (DALY) was assessed based on 2013 Global Burden of Disease estimates.

Results: 1120 children were screened with a mean age of 10.9 years; $46 \%$ were female. A TST induration $\geq 10 \mathrm{~mm}$ was documented in $78(6.9 \%)$ cases, of which $21(1.9 \%)$ were further evaluated. Of those, 17 children were diagnosed as LTBI and none had active TB. The highest rates of positive TST results were found in migrant children from Africa (16.6\%) and Turkey (15.4\%). Screening for LTBI was cost-saving for prevalence rates of $11 \%$ and higher; screening was cost-effective (cost per DALY < gross domestic product (GDP) per capita) for any prevalence of $2 \%$ or higher.

Conclusion: School-based TB screening programs targeting migrant children from high-risk regions not only have the potential to prevent progression to active TB but can also reduce health system cost if LTBI prevalence is sufficiently high. 


\section{P04 \\ Identification of Non-Tuberculosis Mycobacteria in COPD Patients Undergoing Lung Volume Reduction: More Frequent Than Expected?}

$\underline{G . ~ B e r r a ~}^{1}$, J. Plojoux ${ }^{1}$, P.M. Soccal ${ }^{1,2}$, J.-P. Janssens ${ }^{1,2}$

${ }^{1}$ Geneva University Hospitals, ${ }^{2}$ Université de Genève, Geneva, Switzerland

Introduction: Non-tuberculosis mycobacteria (NTM) are more and more frequently isolated in subjects with chronic respiratory diseases. Although this association is epidemiologically well known, specific data on NTM in COPD patients and relationship with prognosis in this specific group are lacking.

Methods: We retrospectively collected bacteriological findings from all bronchial aspirates obtained during bronchoscopy performed for endoscopic lung volume reduction (ELVR) in severe COPD patients. All patients included met eligibility criteria for ELVR, namely: age $<75$ years, ex smoker ( $>6$ months), emphysema, persisting dyspnea despite maximal medical therapy including a recent rehabilitation program, $\mathrm{FEV}_{1}<45 \%$ of predicted value, marked hyperinflation, a 6-minute walk distance $>140$ meters and no or few acute exacerbations during the year prior to the procedure.

Results: NTMs were isolated in $8 / 44$ patients ( $18.2 \%$ of cases). Mycobacterium avium complex (MAC) was most the most prevalent NTM identified (5/8, all M. avium subtype). In addition, one Mycobacterium xenopi, one Mycobacterium gordonae and one Runyon Group IV NTM (rapidly growing, non-pigmented) were isolated.

All specimens were isolated from culture of bronchial aspirates. No sample was microscopy positive. NTM were isolated once in most patients (6/8), twice in one patient and three times in another. With a mean follow up of 993 days, none of the patients required specific antimycobacterial treatment for NTM-associated lung disease, defined as a progression of symptoms or radiological lung lesions or an accelerated lung function decline attributable to NTM infection.

Other pathogens frequently isolated from bronchial aspirates were Haemophilius influenzae (4/44 patients), various Gram negative bacilli (11/44 patients) and Aspergillus species (6/44 patients).

Table 1. (for Abstract P04)

\begin{tabular}{llc}
\hline Variables & $\begin{array}{l}\text { Patients } \\
\text { colonized with } \\
\text { NTMs }(\mathrm{N}=8)\end{array}$ & $\begin{array}{c}\text { Patients without } \\
\text { NTMs }(\mathrm{N}=36)\end{array}$ \\
\hline Patients on OCS, n/N, (\%) & $1 / 8(12.5 \%)$ & $2 / 36(5.5 \%)$ \\
Patients on ICS, n/N, (\%) & $7 / 8(87.5 \%)$ & $31 / 36(86 \%)$ \\
FEV1, mean (SD) & $31.5(39.3-23.8)$ & $31.3(41.7-20-9)$ \\
DLCO, mean (SD) & $47.7(34.8-60.6)$ & $46.2(30.7-61.7)$ \\
6MWT, mean (SD), m & $463(388-537)$ & $348(236-459)$ \\
Diabetes, n/N, (\%) & $1 / 8(12.5 \%)$ & $1 / 36(2.8 \%)$ \\
Immunosuppression, n/N, (\%) & $1 / 8(12.5 \%)$ & $0 / 36(0 \%)$ \\
Chronic renal disease, n/N, (\%) & $0 / 8(0 \%)$ & $1 / 36(2.8 \%)$ \\
\hline
\end{tabular}

Abstracts
Conclusion: In a population of 44 patients with severe COPD undergoing endoscopic lung volume reduction, we identified a significantly higher rate of NTMs than previously reported. The clinical impact of this finding has to been further explored in a prospective study.

\section{P05 \\ A Prediction Model for COPD Exacerbation - Data from the Swiss COPD Cohort Study}

\section{E. Thesenvitz, A. Naduvilekoot, S. Züsli, S. Maier, T. Jendricke, J. Leuppi, T. Dieterle}

Clinical Research, University Clinic of Medicine, Cantonal Hospital Baselland, Liestal, Switzerland

Introduction: It is estimated that about 400,000 patients are affected by COPD in Switzerland. Despite the large prevalence longterm data on symptoms and lung function in this population are lacking. Therefore the goal of the Swiss COPD Cohor study (SCCS) was to assess the situation of COPD care in Switzerland. A further goal was to develop a prediction model for the risk of exacerbation.

Method: Patients with a verified COPD diagnosis (FEV1/FVC $<70 \%$ without reversibility with bronchodilator [improvement of FEV $1<200 \mathrm{ml}$ or $<12 \%$ ]) were included into the study by general practitioners and pneumologists in Switzerland. Clinical and spirometry exams were performed at baseline and every six months thereafter including information about medical and non-medical therapy, COPD Assessment Test (CAT-Score), co-morbidities and exacerbation. Univariate analysis was performed to identify factors statistically associated with the risk of exacerbation. Factors significant in univariate analysis were included into a binary logistic regression model to determine statistically independent predictors and a risk score.

Results: 166 Patients from SCCS recruited between May 2011 and June 2016 were included into this interim analysis. The following Table 1 summarizes statistically independent predictors for exacerbation. A score of $\geq 2$ points was associated with an increased risk $(\mathrm{HR}=2.321,95 \%$ confidence interval (1.186-4.541)).

Conclusion: A score consisting from 5 variables is able to reliably predict the risk of COPD exacerbation within one year.

Table 1. Predictor for a COPD exacerbation within one year (for Abstract P05)

\begin{tabular}{lll}
\hline Predictor & no & yes \\
\hline On-going cancer & 0 & 1 \\
Previous exacerbation & 0 & 1 \\
CAT-Score $>12$ & 0 & 1 \\
mMRC $>0$ & 0 & 1 \\
BMI $<21$ & 0 & 1 \\
\hline
\end{tabular}




\section{P06 \\ The Use of Combination Therapy in the Swiss COPD Cohort Study - A Cross-Sectional Analysis}

\author{
E. Thesenvitz, S. Züsli, A. Naduvilekoot, S. Maier, T. Jendricke, \\ J. Leuppi, T. Dieterle \\ Clinical Research, University Clinic of Medicine, Cantonal \\ Hospital Baselland, Liestal, Switzerland
}

Introduction: It is estimated that about 400,000 patients are affected by COPD in Switzerland. Despite the large prevalence long-term data on symptoms and lung function in this population are lacking. Therefore the goal of the Swiss COPD Cohort Study SCCS was to assess the situation of COPD care in Switzerland with a particular focus on medical therapy related to GOLD classification.

Method: Patients with a verified COPD diagnosis (FEV1/FVC $<70 \%$ without reversibility with bronchodilator [improvement of FEV $1<200 \mathrm{ml}$ or $<12 \%$ ]) were included into the study by general practitioners and pneumologists in Switzerland. Clinical and spirometry exams were performed at baseline and every six months thereafter including information about medical and nonmedical therapy, COPD Assessment Test (CAT-Score) and comorbidities.

Results: 164 Patients from SCCS recruited between May 2011 and June 2016 were included into this interim analysis. Data on current medication is summarized in the following Table 1 .

Conclusion: This interim analysis of COPD cohort demonstrates that combination therapy is widely used in patients GOLD class III and IV. However, the use of combination therapy in COPD GOLD IV is less frequent than expected.

\section{P07 \\ Towards Standardized Follow-Up Care for Patients with Primary Ciliary Dyskinesia (PCD)}

\author{
M. Goutaki ${ }^{1,2}$, F. Halbeisen ${ }^{1}$, M. Martin', C. Hogg ${ }^{3}$, C. Casaulta ${ }^{2}$, \\ I. Amirav ${ }^{4}$, J. Barben ${ }^{5}$, E. Moya ${ }^{6}$, K. Nielsen ${ }^{7}$, G. Thouvenin ${ }^{8}$, \\ J. Lucas $^{9}$, C. Kuehni ${ }^{1}$
}

${ }^{1}$ Institute of Social and Preventive Medicine, ${ }^{2}$ Paediatric Respiratory Medicine, Children's University Hospital of Bern, University of Bern, Bern, Switzerland, ${ }^{3}$ Department of Paediatrics, Primary Ciliary Dyskinesia Centre, Royal Brompton and Harefield Foundation Trust, London, United Kingdom, ${ }^{4}$ Department of Pediatrics, Faculty of Medicine, Bar Ilan University, Safed, Israel, ${ }^{5}$ Division of Paediatric Pulmonology, Children's Hospital St. Gallen, St. Gallen, Switzerland, 'Division of Services for Women and Children, Women's and Newborn Unit Bradford Royal Infirmary, University of Bradford, West Yorkshire, United Kingdom, ${ }^{7}$ Danish PCD Centre Copenhagen, Paediatric Pulmonary Service, Copenhagen University Hospital, Copenhagen, Denmark, ${ }^{8}$ Paediatric Pulmonary Department, Trousseau Hospital APHP, Sorbonne Universities and Pierre et Marie Curie University, Paris, France, ${ }^{9}$ Primary Ciliary Dyskinesia Centre, NIHR Biomedical Research Centre, University of Southampton, Southampton, United Kingdom

Introduction: Reporting of clinical information in medical charts is not standardized; thus data collected retrospectively from records is of little value for research. A recent systematic review (Goutaki et al, ERJ 2016), found that assessment of clinical symptoms of patients with PCD varied considerably between studies making comparisons impossible. Management and follow-up differ between countries, and is usually extrapolated from other diseases. We aimed to develop proformas for longitudinal data collection during routine care, allowing standardized reporting of frequency and severity of clinical signs and symptoms.

Methods: We collected available proformas for management of PCD and related diseases (e.g. cystic fibrosis) in different countries from the BEAT-PCD network. We compared them with the available and designed a new harmonized proforma. We discussed several versions within a large multidisciplinary group of $>30$ collaborators (including paediatric and adult pulmonologists, ENT specialists and diagnostic experts) and patient representatives to improve the content and the structure via an adapted Delphi process.

Table 1. The use of combination therapy (for Abstract P06)

\begin{tabular}{|c|c|c|c|c|}
\hline $\begin{array}{l}\text { GOLD Classification } \\
\mathrm{N}=164\end{array}$ & $\begin{array}{l}\mathrm{I} \\
24\end{array}$ & $\begin{array}{l}\text { II } \\
73\end{array}$ & $\begin{array}{l}\text { III } \\
55\end{array}$ & $\begin{array}{l}\text { IV } \\
12\end{array}$ \\
\hline Short-acting Bronchodilators & $4(16.7)$ & $24(32.9)$ & $33(60.0)$ & $6(50.0)$ \\
\hline ICS & $2(8.3)$ & $11(15.1)$ & $22(40.0)$ & $5(41.7)$ \\
\hline Single compounds or combination of LABA and ICS & $7(28.2)$ & $33(45.2)$ & $44(80.0)$ & $6(50.0)$ \\
\hline LAMA and LABA as single compounds or as combination & $3(12.5)$ & $17(23.3)$ & $25(45.5)$ & $5(41.7)$ \\
\hline
\end{tabular}


Table 1. Standardised PCD proforma modules (for Abstract P07)

Module 1 Basic patient information, diagnostic evaluation and baseline medical history

Module 2 Medical history (reported symptoms)

Module 3 Physical examination of lungs and heart

Module 4 Physical examination of Ear Nose Throat (ENT)

Module 5 Growth measurements and clinical tests (lung function, imaging, microbiology)

Module 6 Treatment (hospitalization, surgeries, medication and physiotherapy)

Module 7 Enviromental exposures and lifestyle

Results: The new standardized proforma consists of 7 thematic modules (Table 1) and includes key questions about signs, symptoms, clinical tests, treatments and environmental exposures. As a next step, we will pilot the proforma in paediatric and adult PCD centres, before finalizing it. Consenting centres will be able to enter the data collected in a standardised way to an online database to be used for prospective research. Collected data will be linked to existing databases allowing for data exchange and efficient data use.

Conclusion: Disease-specific and standardized follow-up can improve the quality of information collected for clinical care and research by adding standardized prospective longitudinal information from routine visits. This will be a step towards standardized care for PCD and enable using valuable data from routine care for research studies.

Funding: Lung League Bern, SNF 32003B_162820, COST BEAT-PCD BM1407.

\section{P08 \\ Tobacco Taxes in Switzerland: Losses for Public Health - Gains for the Industry}

\section{R.M. Kaelin P. Diethelm}

Oxyromandie/OxySuisse, Geneva, Switzerland

Introduction: Decisions on Tobacco (T) Taxes (TX) are Public Health (PH) measures, if applied correctly: A. TX, following WHOconvention, should amount to $75 \%$ of retail prices (RP),resulting (B) in high RP to prevent T-uptake by youth; C. RP- increases should occur by steps of $>10 \%$, to curb consumption. We searched, if cigarette (cigs) TX increases 2003-2013 were positive for $\mathrm{PH}$.

Methods: Analysis of RP, TTX,comparison with EU-countries. Results:

1. Different T-products are not taxed coherently: Cigs are taxed $61 \%$ of RP(VATincl.), pipe and heat not burn T only $19 \%$. Cheap pipe $\mathrm{T}$ is thus used for handmade cigs, e.g.: "Marboro" pipe T, RP $6.50 / 30 \mathrm{~g}$ (1.25.CHF TX) yields 40-60 cigs at $<$ half the RP of industrial "Marlboro" cigs at 8.-CHF (TX 5 CHF) for a pack of 20.
Also, one day pack of "Marlboro"heat not burn T at 8.-CHF(TX $0.95 \mathrm{CHF}$ ) leaves 7.-CHF to to industry.

2. Gains of the indusry on a pack of industrial cigs in Switzerland are 3.14 CHF, about the double that of EU-countries.

3 . If the rate of $75 \% \mathrm{RP}$ had been applied, the TX income in 2012 2016 would have been increased by 352-428 Million CHF per year.

4. The RP of cigs rose from 4.-to 8.- CHF in 2003-2013, but TX increases were so small, that industry could increase RP by steps of $<10 \%$, in order not to curb consumption significantly keeping gains high.

Conclusions: Tobacco Taxes in Switzerland serve more the industry than Public Health. They were implemented as to result in too low retail prices compared to other countries, thus preventing to curb consumption. This allowed industry to keep gains high. The huge Tax difference between pipe/heat not burn Tobacco compared to industrial cigarettes fosters youth smoking and provides big gains on the newer products.

\section{P09 \\ Development of Hospitalizations for Pulmonary Hypertension in Switzerland from 2002 to 2015}

\section{F. Aigner, F. Baty, M. Brutsche}

Department of Pulmonary and Sleep Medicine, Kantonsspital St. Gallen, St. Gallen, Switzerland

Introduction: The objective of the present study was to analyze the development of hospitalizations for pulmonary hypertension in Switzerland.

Methods: The data set was provided by the Swiss Federal Office for Statistics, which offers a nation-wide coverage of all hospitalized patients using the ICD-10 coding system. The WHO-Classification of pulmonary hypertension is not used in this coding system. We analyzed the number of hospitalizations as main diagnosis for overall pulmonary hypertension (I27), pulmonary arterial hypertension (PAH; I27.0), chronic thromboembolic pulmonary hypertension (CTEPH; I27.20), other specified pulmonary-associated heart diseases and Cor pulmonale (I27.8 and I27.9) and not otherwise specified secondary pulmonary hypertension (I27.28) from 2002-2015. The development of age and comorbidities in patients with PAH was analyzed.

Results: Overall, the incidence of hospitalizations for all causes of pulmonary hypertension did not change (average of 450 cases per year) since 2002. Since 2009, we observed a sudden significant decrease in the incidence of hospitalizations for $\mathrm{PAH}$, independent of the canton (from 255 per year in 2008 to 82 per year in 2015). At the same time coding for CTEPH and not otherwise specified secondary pulmonary hypertension was introduced. Since then the incidence in these newly introduced categories significantly increased (from 0 cases in 2009 to 256 in 2015). There was no difference in age and cardiovascular comorbidities seen in patient with $\mathrm{PAH}$.

Conclusions: Since the incidence of the overall hospitalizations for pulmonary hypertension remain stable, we assume that the profound decline of hospitalizations for $\mathrm{PAH}$ is due to a change in coding practices in 2009 and not due to an increase in availability of vasodilatory therapy PAH. 


\section{P10 \\ Echinacea for the Prevention of Respiratory Tract Infections in Children 4-12 Years: A Randomized, Blind and Controlled Study}

M. Ogal ${ }^{1}$, P. Klein ${ }^{2}$, R. Schoop ${ }^{3}$

${ }^{1}$ Pediatric Clinic, Brunnen, Switzerland, ${ }^{2}$ d.s.h. statistical services $\mathrm{GmbH}$, Rohrbach, Germany, ${ }^{3}$ Medical Department, Bioforce AG, Roggwil, Switzerland

Withdrawn.

\section{Poster Walk 2: Clinical Lung / Patient Management 1}

P11

\section{Pyogenic Lung Abscesses: The Place of Conservative Treatment}

\section{Udry' ${ }^{1}$, A. Ogna' 1 , M. Gonzalez ${ }^{2}$, M. Oriol' ${ }^{3}$, L. Nicod}

${ }^{1}$ Service of Pulmonology, ${ }^{2}$ Service of Thoracic Surgery, ${ }^{3}$ Service of Infectious Diseases, Centre Hospitalier Universitaire Vaudois, Lausanne, Switzerland

Introduction: Lung abscesses (LA) have a low incidence but still carry a 5\% mortality. As bronchoaspiration is a major cause of LA, they are mostly due to anaerobic bacteria. Medical treatment (MT) by antibiotics successfully cures the LA in $80-90 \%$ of cases. More invasive treatments such as surgical resection (SR) may be attempted. There are currently no clear guidelines for surgical indication, but SR is generally recommended if MT fails or for complicated LA.
In this study, we tried to identify objective arguments to choose between MT and SR as the first line treatment in a given patient.

Methods: we did a retrospective analysis of medical records of 68 patients treated for LA at our institution between 2013-2015. The patients were divided in two groups: 42 patients treated by MT and 22 patients treated by SR. We compared baseline characteristics and outcomes between the two groups.

Results: The median duration of antibiotic treatment was 52 days $[37.25 ; 61]$ in SR and $50[36.75 ; 86.75]$ in MT (p > 0.05). Mean length of hospital stay was equivalent between the two groups: $23.64 \pm 16.11$ days in SR and 23.85 \pm 19.03 in MT $(p>0.05)$. Cases of complicated LA with empyema were significantly higher in SR $(77.3 \%)$ than in MT $(17.4 \%, \mathrm{p}<0.0001)$. There was no difference neither in the LA size $(4.49 \pm 2.66 \mathrm{~cm}$ in SR vs $5.22 \pm 2.43 \mathrm{~cm}$ in MT, p > $0.05)$ nor in the microbiologic repartition. The mortality rate was similar between the two groups: $4.5 \%$ in SR and $8.7 \%$ in MT(p >0.05).

Conclusion: MT and SR have similar rate of mortality and morbidity. MT must always be considered as first line treatment. SR is indicated in MT failure. However, SR indication in complicated LA seems less evident, as some patients heal despite antibiotic treatment alone. Conservative treatment most often leads to good lung tissue healing, even in large cavities.

\section{P12 \\ Patients Rejected for Lung Transplantation: Who Are They?}

\section{A. Lenoir ${ }^{1}$, C.R. Perez Valdes ${ }^{1,2}$, J.-D. Aubert ${ }^{1}$, On behalf of the Lausanne-Geneva lung transplantation programme}

${ }^{1}$ Service de Pneumologie, Centre Hospitalier Universitaire Vaudois, Lausanne, ${ }^{2}$ Hôpital de Rolle, Rolle, Switzerland

Listing a patient for lung transplantation is a critical decision given this treatment's limited availability. The International Society for Heart and Lung Transplantation published in 2015 a consensus
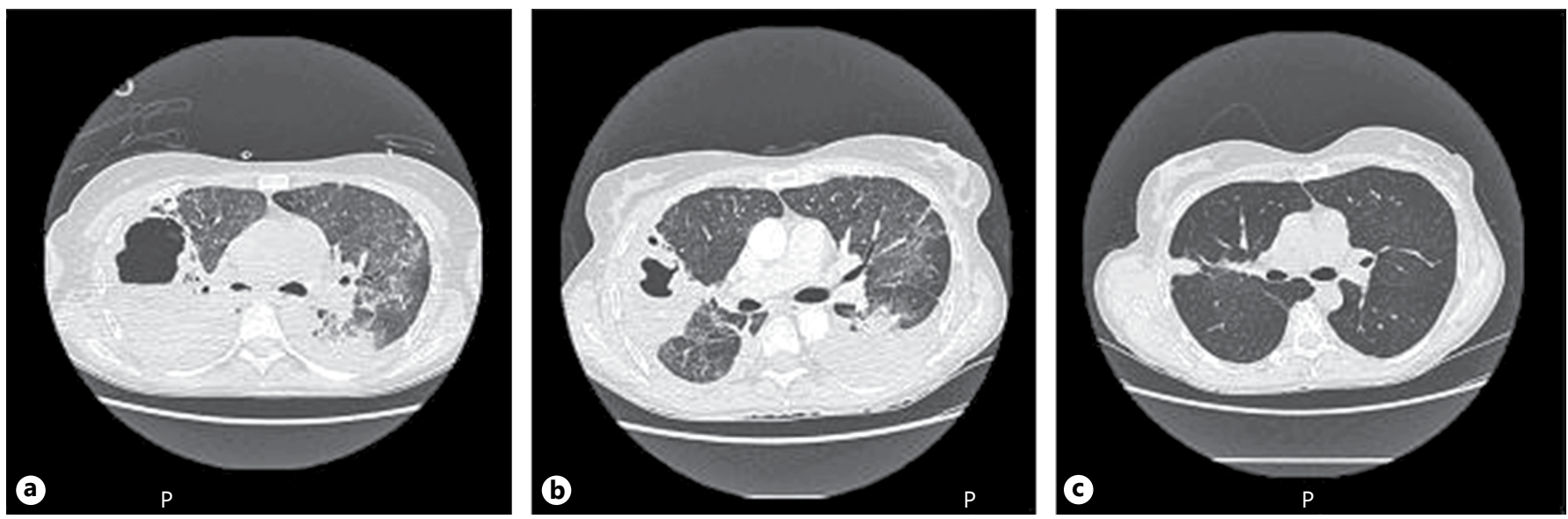

Fig. 1. Right superior lobe lung abscess complicated with empyema (a) and the evolution at 1 month (b) and 4 months (c) with a conservative treatment (for Abstract P11). 
document as decision guidance for selecting lung transplant candidates. However, a detailed analysis comparing patients placed on the waiting list to those deemed unsuitable has rarely been made. We retrospectively evaluated lung recipient candidates referred to our centre regarding diagnosis, final decision and outcomes.

Demographics, respiratory diagnosis, and the board's decision were recorded for all patients referred to the Lausanne-Geneva lung transplant board from $04 / 2004$ to $07 / 2017$. If rejected, the primary contraindication or the combination of relevant contraindications was determined. Furthermore, survival on/off the waiting list was calculated.

Among 363 referred candidates for lung transplant, 97 (26.7\%) were rejected. Median age (range) was higher in non-listed patients: $61.5(12-68)$ vs 53.2 (14-65) years. Acceptance rate was $90.2 \%$ among cystic fibrosis patients, $74.0 \%$ among idiopathic pulmonary fibrosis patients, $69.0 \%$ among chronic lung allograft dysfunction (CLAD) patients, $67.6 \%$ among COPD patients and $74.1 \%$ among other diagnostic categories. Main contraindications for listing were cardiovascular (17.5\%), insufficient severity of the respiratory condition (15.5\%), age (13.4\%) and multiple organ dysfunction (12.4\%). Pooled other causes accounted for $23.8 \%$, including cancer, multiple comorbidities, surgical inoperability, psychosocial difficulties and inappropriate indications. A combination of causes constituted $17.5 \%$. One-year-survival differed significantly between listed and non-listed patients: $88 \%$ vs $79 \%(\mathrm{p}=0.0001)$. This difference increased at three years: $80 \%$ vs $57 \%$, with over $80 \%$ of listed patients meanwhile transplanted. Mortality on the waiting list was $12 \%$.

Patients rejected for lung transplant tend to be older and rather suffer from COPD or CLAD than accepted candidates. Main contraindications leading to rejection in our centre were cardiovascular, premature referral and age; a combination of various contraindications was common. Listed patients had a clear survival benefit.

\section{P13 \\ Sensitivity and Specificity of EGFR Liquid Biopsy versus Serum Carcinoembryonic Antigen in Patients with Adenocarcinoma of the Lung}

\section{S. Saenghirunvattana ${ }^{1}$, P. Saenghirunvattana ${ }^{2}$, C. Gonzales ${ }^{1}$, K. Suthisri ${ }^{1}$, C. Siangproh ${ }^{1}$}

${ }^{1}$ Pulmonary and Chest Center, Bangkok Hospital Medical Center, Bangkok Dusit Medical Services, ${ }^{2}$ Chulalongkorn University, Bangkok, Thailand

Introduction: Tissue biopsy is necessary to diagnose lung cancer but it becomes impractical due to its invasive nature and complications. A new test, epidermal growth factor receptor (EGFR) liquid biopsy provides wider range of information obtained from blood samples that may help clinicians into better personalized treatment particularly in cases of adenocarcinoma of the lung.

The aim of this retrospective study is to compare which among EGFR liquid biopsy and carcinoembryonic antigen (CEA) has higher predictive values in patients with adenocarcinoma of the lung.
Methods: Thirty patients with lung nodules on their chest CT scan underwent diagnostic tests: EGFR liquid biopsy, serum CEA, tissue biopsy and/ or surgery between September 2016 and November 2017. Data gathered were patient age, gender, smoking history, serum CEA levels, EGFR liquid biopsy results and final diagnosis. EGFR liquid biopsy result was positive when EGFR mutation is detected. The sensitivity, specificity, positive predictive and negative predictive values of CEA and EGFR liquid biopsy were compared based on data collected.

Results: There were thirty patients ( $27 \%$ male and $73 \%$ female) with mean age of 60 years old. Smokers were $20 \%$, and non-smokers: $80 \%$. Pathological diagnoses were: adenocarcinoma: $37 \%$ and others were: tuberculosis: $47 \%$, benign cases: $7 \%$, squamous metaplasia: $3 \%$, anaplastic giant cell carcinoma: $3 \%$ and carcinoid tumor at $3 \%$.

EGFR liquid biopsy rates in diagnosing Adenocarcinoma are: sensitivity: $85.7 \%$, specificity: $100 \%$, positive predictive value: $100 \%$ and negative predictive value is $94.7 \%$. Meanwhile, CEA rates in detecting Adenocarcinoma are: sensitivity: $69.2 \%$, specificity: $88 \%$, positive predictive value: $81.8 \%$ and negative predictive value is $78.9 \%$.

Conclusion: Among cases of adenocarcinoma of the lung, liquid biopsy provides more specific, accurate data and has a higher predictive rate and yield compared to CEA.

\section{P14 \\ Adherence to COPD GOLD 2017 Guidelines in Swiss Pulmologists and General Practitioners}

J.L. Marmy ${ }^{1}$, J. Diedrich ${ }^{2}$, T. Dieterle ${ }^{1}$, J.D. Leuppi ${ }^{1}$

${ }^{1}$ University Clinic of Medicine, Cantonal Hospital Baselland, Liestal, ${ }^{2}$ Boehringer Ingelheim (Schweiz) GmbH, Basel, Switzerland

Background: A major revision of the Global Initiative for Chronic Obstructive Lung Disease (GOLD) guidelines on chronic obstructive pulmonary disease (COPD) was published 2017.

Objective: To explore loyalty to GOLD 2017 amongst pulmologists and general practitioners.

Methods: This questionnaire-based survey was approved by ethics committee. 92 physicians from all over Switzerland reported demographics, exacerbation history, dyspnoea status and current therapy of their COPD patients. Patients were sorted into the GOLD 2017 treatment gird by exacerbation status and symptoms. A correct therapy for GOLD A was defined as a therapy with a short-acting beta-agonist (SABA), or short-acting muscarinic-antagonist (SAMA). For GOLD B and C, either a long-acting betaagonist (LABA), long-acting muscarinic-antagonist (LAMA), or LAMA/LABA combination was judged as acceptable treatment. The use of inhaled corticosteroid (ICS) was valued incorrect for GOLD groups A to C. In GOLD D a correct therapy was at least the use of a LAMA/LABA combination.

Results: In total 511 Patients, 288 (56.4\%) men and 223 (43.6\%) women, were enrolled. 140 (27.4\%) patients were grouped GOLD A, 184 (36.0\%) GOLD B, 61 (11.9\%) GOLD C and 118 (23.1\%) GOLD D. Dyspnoea was reported in $319(62.5 \%)$ patients (mMRC 
$\geq 2$ ). In GOLD A, 122 (87.1\%) patients were over-treated with a LABA, LAMA, ICS or combination therapy. 96 (52.2\%) GOLD B patients were treated correct, $68(37.0 \%)$ were over-treated. In GOLD C, 30 (49.2\%) patients were treated correct, and 29 (47.5\%) patients were over-treated. GOLD D grouped patients were treated best, as $93(78.8 \%)$ patients receiving a therapy in accordance with the guidelines. Nevertheless, 18 (15.2\%) GOLD D patients were undertreated.

Conclusion: Although $57.6 \%$ of the participating physicians were pulmologists, half of the COPD patients are over-treated by ICS. Further educational efforts by the society are needed to get patients on adequate treatment.

\section{P15 \\ Effect of Inpatient Treatment at the Hochgebirgsklinik Davos Located at 1600 m Elevation on Patients with Chronic Obstructive Pulmonary Disease}

\author{
L. Kraus ${ }^{1}$, G. Menz ${ }^{1}$, T. Bieber ${ }^{2}$, H.-W. Duchna \\ ${ }^{1}$ Hochgebirgsklinik Davos, Davos-Wolfgang, Switzerland, \\ ${ }^{2}$ Dermatologie, Universität Bonn, Bonn, Germany
}

Introduction: Rehabilitation therapy plays an important role in the treatment of chronic obstructive pulmonary disease (COPD). The benefit of climate therapy in high altitude in these patients is a matter of controversy in literature. Therefore, the impact of the combination of multimodal rehabilitation and Davos' climatic conditions on patients with COPD were investigated in the present study.

Methods: In a prospective observational study 49 subjects were examined at baseline and at the end of their inpatient stay at the Hochgebirgsklinik Davos. Means of the analysis were a carefully structured anamnesis, pulmonary function test, performance test and a validated disease-specific subjective COPD assessment test (CAT).

Results: During the inpatient rehabilitative treatment with an average duration of 16.45 days the results of the CAT improved significantly from 20.15 to 15.54 points $(\mathrm{p}=0.006)$. In pulmonary function, the following parameters changed significantly: $\mathrm{FEV}_{1}$ increased from $1.35 \mathrm{~L}$ to $1.39 \mathrm{~L}$. $(\mathrm{p}=0.011), \mathrm{VC}$ from $2.61 \mathrm{~L}$ to 2.70 $\mathrm{L}(\mathrm{p}=0.004)$ and PEF from $3.45 \mathrm{~L} /$ second to $3.93 \mathrm{~L} /$ second $(\mathrm{p}=$ 0.002 ). RV reduced significantly from $211.53 \%$ to $194.32 \%$ ( $\mathrm{p}=$ 0.046). Physical performance measured in ergometry improved significantly from $35.83 \mathrm{~W}$ to $48.75 \mathrm{~W}$ ( $\mathrm{p}=0.007$ ) as well as the distance in the 6-min walk test, which rose from $303.64 \mathrm{~m}$ to 331.90 $\mathrm{m}(\mathrm{p}=0.009)$.

Conclusion: The results of the study demonstrate that patients with COPD clearly benefit from an inpatient rehabilitation at the Hochgebirgsklinik Davos. Essential parameters of lung function and physical performance improved as well as the subjective state of health. The strongest effect, respectively the greatest clinical significance appeared in physical performance and CAT. It is remarkable that COPD patients benefit from the combination of mountain climate and multimodal rehabilitation despite the limited reversibility of their bronchial obstruction and the altered oxygen conditions of altitude.

\section{P16 \\ Objective Adherence to Inhaled Medication and Health-Related Outcomes in Asthma and Chronic Obstructive Pulmonary Disease after an Electronic Monitoring Intervention}

\author{
C. Gregoriano $^{1,2}$, T. Dieterle ${ }^{1,3}$, A.-L. Flamm¹, S. Dürr' ${ }^{1}$, S. Maier ${ }^{1}$, \\ I. Arnet $^{2}$, K.E. Hersberger ${ }^{2}$, J.D. Leuppi ${ }^{1,3}$ \\ ${ }^{1}$ University Department of Medicine, Cantonal Hospital \\ Baselland, Liestal, ${ }^{2}$ Department of Pharmaceutical Sciences, \\ ${ }^{3}$ Faculty of Medicine, University of Basel, Basel, Switzerland
}

Introduction: Poor medication adherence is common in asthma and COPD patients, resulting in reduced health outcomes and increased healthcare-costs. Sparse data are available on the impact of targeted interventions to improve adherence to inhaled medication. The aim of this study was to investigate the impact of an acoustic reminder and a close supervision on adherence to inhaled medication and on course of disease and quality of life (Qol) in asthma and COPD patients.

Methods: In this single-blinded trial, asthma and/or COPD patients were randomly assigned either to the intervention or the control group. Adherence to inhaled medication was monitored using electronic data capture devices, recording date and time of each inhalation device actuation. Follow-up was six months. Primary outcome was defined as "time to next exacerbation". Secondary outcomes included number of exacerbations, number of exacerbations with hospitalization, taking/timing adherence, and Qol during follow-up.

Results: Time to first exacerbation was longer (172 days [ $95 \%$ CI, 161 to 182 ] vs. 161 days [95\% CI, 149 to 174 ],$p=0.27)$ and the risk for experiencing an exacerbation lower (HR, 0.67 [95\% CI, 0.36 to 1.33 ],$p=0.14$ ) in the intervention compared to the control group. Significantly more days with a taking adherence of 80 $100 \%$ were observed in the intervention compared to the control group with puff inhalers $(81.6 \pm 14.2 \%$ vs. $60.1 \pm 30.3 \%, p<0.001)$ and dry powder capsules $(89.6 \pm 9.8 \%$ vs. $80.2 \pm 21.3 \%, p=0.01)$. In addition, timing adherence in patients using puff inhalers was significantly higher in the intervention group $(68.9 \pm 25.0 \%$ vs. $50.6 \pm 32.5 \%, p<0.001)$.

Conclusion: Significantly improved taking and timing adherence were observed in the intervention compared to the control group. This was associated with a trend towards a longer time to next exacerbation and lower incidence of exacerbations, indicating that regular automatic personalized reminders may have beneficial effects on adherence and outcomes in asthma and COPD patients. 


\section{P17 \\ Viral Detection in COPD - Implication in Exacerbations}

D. Stolz ${ }^{1}$, H. Hirsch ${ }^{2}$, D. Schilter ${ }^{3}$, R. Louis ${ }^{4}$, J. Rakic ${ }^{1}$, L. Boeck' ${ }^{1}$, E. Papakonstantinou' ${ }^{1}$, C. Schindler ${ }^{2,5}$, L. Grize ${ }^{2,5}$, M. Tamm $^{1}$

${ }^{1}$ Pneumology, ${ }^{2}$ Department of Biomedicine, University of Basel, Basel, ${ }^{3}$ Lindenhof Hospital, Bern, Switzerland, ${ }^{4}$ Pneumology, University of Liege, CHU Liege, Belgium, ${ }^{5}$ Swiss Tropical and Public Health Institute, Basel, Switzerland

Background: Viral respiratory tract infections have been implicated as the predominant risk factor associated with acute exacerbations of COPD (AECOPD). We aimed to evaluate the incidence of viral infection and its association to AECOPD.

Methods: 450 patients with stable, moderate to very severe COPD, were followed for a mean of 27 months. Detection of 18 viruses (Adenovirus, Influenza A-B-H1-H3, Parainfluenza 1-4, Respiratory Syncytial virus (RSV) A-B, Rhinovirus/enterovirus, Coronavirus NL63, -OC43, -229E, -HKU1, Bocavirus, Metapneumovirus) was performed in naso- and oropharyngeal swabs that were collected at URTI onset $(\mathrm{n}=391), 10$ days thereafter $(\mathrm{n}=$ $356)$, at exacerbations $(n=177)$ and stable periods $(n=1909)$ using a commercial multiplex nucleic acid amplification testing, adding up to over than 50,000 individual viral PCRs.

Results: Evidence of at least one respiratory virus was significantly more common at exacerbation $(39.0 \%, P<0.001)$, at URTI onset $(52.7 \%, P<0.001)$, and at 10 days following an URTI $(15.2 \%$, $P<0.001$ ), as compared to the stable period of the disease $(5.4 \%)$. Rhinovirus was the most frequently detected virus $(35.7 \%$ of all viruses at exacerbation). However, only $11 \%$ of all rhinoviruses' detections were associated with exacerbation. Other viruses such as metapneumovirus, influenza viruses and respiratory syncytial virus were more frequently exacerbation-related. Patients presenting a positive viral PCR at URTI onset did not have a higher incidence of exacerbation, as compared to those showing negative viral PCR results $(14.6 \%$ versus $14.6 \%, P=0.993)$. Detection of parainfluenza 3 at URTI onset was associated with a significantly higher risk of exacerbation as compared to the other viruses (HR 6.24, 95\% CI 1.85-21.0, $P=0.003$ ) and this risk remained significant after adjustment for the treatment effect.

Conclusion: The prevalence of viral infection at the stable period of COPD is low. The risk of exacerbation following the onset of URTI symptoms depends on the particular virus associated with the URTI.

\section{P18 \\ Oral Triple Combination Therapy in Pulmonary- Arterial Hypertension and Chronic Thromboembolic Pulmonary Hypertension: Data from the Zurich Cohort}

C. Berlier E. Schwarz, S. Saxer, M. Lichtblau, S. Ulrich

Pulmonary Division, University Hospital of Zurich, Zurich, Switzerland

Introduction: Patients with pulmonary arterial hypertension $(\mathrm{PAH})$ and chronic thromboembolic pulmonary hypertension (CTEPH) may benefit from early triple disease-targeted medical combination therapy since the availability of the oral selective prostacyclin-receptor agonist Selexipag. We reviewed Zürich cohort patients who received Selexipag in a triple therapy.

Methods: We reviewed Zürich cohort patients diagnosed with PAH or CTEPH who received Selexipag. New York Heart Association (NYHA) functional class, 6-min walk distance (6MWD) and NT-pro-BNP were retrieved before and at regular follow-up under triple therapy.

Results: To date, 14 patients with PAH (12)/CTEPH (2), 11 females, mean \pm SD age $57.2 \pm 12.5$ years were followed for a mean of $195 \pm 120$ days. 4 patients discontinued Selexipag ( 2 change to intravenous prostanoids, 2 side effects). The mean dose achieved was $2160 \pm 847 \mathrm{mcg}$. There were no deteriorations in 6MWD (before $441 \pm 108 \mathrm{~m}$, after $447 \pm 68 \mathrm{~m}, \mathrm{p}=0.19$ ), desaturation at peak $6 \mathrm{MWD}\left(\mathrm{SpO}_{2}\right.$ before $84 \pm 11 \%$, after $\left.77 \pm 16 \%, \mathrm{p}=0.71\right)$, NYHA class (before $2.7 \pm 0.2$, after $2.5 \pm 0.2, \mathrm{p}=0.65$ ) or NT-pro-BNP (before $646 \pm 263 \mathrm{ng} / \mathrm{l}$, after $701 \pm 227 \mathrm{ng} / \mathrm{l}, \mathrm{p}=0.612$ ).

Conclusions: Triple oral combination therapy including Selexipag was generally well supported and contributed to stabilize patients in the NYHA functional class, 6MWD and NT-pro-BNP.

\section{P19}

\section{Accidental Decannulation Following Tracheostomy as Life-Threatening Event on a PICU}

\author{
K. Hoyler' ${ }^{1}$, C. Schlegel-Wagner ${ }^{2}$, K. Ganassi ${ }^{3}$, K. Schwendener ${ }^{3}$, \\ P. Szavay ${ }^{4}$, M. Stocker ${ }^{3}$, N. Regamey ${ }^{1}$ \\ ${ }^{1}$ Pediatric Pneumology, Children's Hospital Lucerne, \\ ${ }^{2}$ Otorhinolaryngology, Lucerne Cantonal Hospital, ${ }^{3}$ Intensiv \\ Care Unit, ${ }^{4}$ Pediatric Surgery, Children's Hospital Lucerne, \\ Lucerne, Switzerland
}

Introduction: In patients with recent tracheostomy accidental decannulation may be life-threatening if the cannula cannot be rapidly reinserted.

Case Report: We report on a patient who presented with severe hoarseness, blue spells and desaturation episodes. Bronchoscopy revealed a glottic to subglottic stenosis grade III (Myer-Cotton) due to a laryngeal web type IV. Tracheostomy was performed at the age of 3 weeks in order to bridge time until a laryngoplasty could be performed. 
After surgery the spontaneously breathing infant was allowed on his mother's lap to comfort his postoperative distress. Within several hours he then developed increasing swelling and skin-emphysema before he rapidly deteriorated. Resuscitation and conventional intubation was performed until the cannula was correctly reinserted. During resuscitation the infant developed bilateral pneumothorax and severe hypoxemia. Brain MRI performed 4 days later showed signs of diffuse hypoxic brain damage. Neurological assessment 6 months later showed normal psychomotor development but a cerebral movement disorder of the right arm.

Conclusion: Accidental decannulation within the first week following tracheostomy is a severe complication that may occur, especially after inadequate mobilisation. It should be anticipated by the staff in charge and necessitates $24 / 7$ availability of a surgeon-on-call, who is familiar with tracheostomy and (re-)insertion of the cannula. A detailed handover after surgery with a written plan for accidental cannulation can further improve the readiness of the intensive care team for this potential life-threatening event. Our case together with two similar cases from other institutions in Switzerland subsequently led to a change of recommendations regarding postoperative care of children undergoing tracheostomy in our institution.

\section{P20 \\ Allergic Bronchopulmonary Aspergillosis (ABPA) Description of Typical Exacerbation. Classical Remission Induction and Implementation of Mepolizumab}

\section{P. Kubena, H.-W. Duchna, G. Capova, G. Menz}

Pneumologie/Allergologie, Hochgebirgsklinik Davos, DavosWolfgang, Switzerland

Introduction: Allergic bronchopulmonary aspergillosis (ABPA) is the most common allergic bronchopulmonary mycosis in humans. ABPA is caused by complex immune-inflammatory reactions to Aspergillus fumigatus allergens.

Five different stages of ABPA are distinguished.

Acute stage therapy is based on systemic corticosteroids.

Case Report:

History: Female patient (58 years), ABPA diagnosis in 2001. 3-4 exacerbations/year. Prednisolone 5 to $7,5 \mathrm{mg} /$ day. Attempts to reduce prednisolone below $5 \mathrm{mg}$ /day resulted in worsening of disease.

Other medication: ICS, LABA, temporary itraconazole. 9/2015 omalizumab, $450 \mathrm{mg}$ every 2 weeks, later every 3 weeks. No positive effect and stop.

Findings: 31.7 .2017 : Routine examination, stable pat. Prednisolone $5 \mathrm{mg} /$ day, IgE $630 \mathrm{kU} / \mathrm{l}$, Eosinophils $0.59^{*} 10^{3} / \mathrm{mm}^{3}$.

The patient then travelled for holiday to Iceland and Greenland. It was cold and wet. Mould exposure was certainly given.

12.9.2017: Presentation: Worsening of clinical picture: copious secretions, cough, tightness in chest, laboured breathing. Crackles ventral apical. X-ray: infiltration left apical ventral.

Lab: IgE $2242 \mathrm{kU} / \mathrm{l}$, Eosinophils $15.5 \%$, absolute $1.31^{*} 10^{3} / \mathrm{mm}^{3}$, $\mathrm{ECP}>200 \mu \mathrm{g} / \mathrm{L}$.
Diagnosis: Exacerbation of ABPA (stage III).

Classical therapy in exacerbation: Remission induction with systemic steroids - Prednisolone $50 \mathrm{mg}$ /day, cefuroxime, drainage of secretions.

15.9.2017: Clinical improvement. Eosinophils $1.7 \%, 0.17^{*} 10^{3} /$ $\mathrm{mm}^{3}$. Reduction of prednisolone.

19.9.2017: Start with mepolizumab to maintain remission and to allow faster tapering of corticosteroids.

Mepolizumab given every four weeks (100 mg subcutan). After the third injection of Mepo we could reduce prednisolone to 2,5 $\mathrm{mg} /$ day. This dose has been proved to be too low in the past and always resulted in exacerbation.

With Mepolizumab the patient remained clinical stable with normal lung function, low eosinophil count $\left(0,1^{*} 10^{3} / \mathrm{mm}^{3}\right)$ and relatively low IgE $720 \mathrm{kU} / \mathrm{l}$ (15.01.2017).

There will be a regular follow up including clinical examination, lung function, IgE, Eosinophils and ECP.

Conclusion: The implementation of mepolizumab for maintaining remission stage of ABPA seems to be successful.

\section{P21 \\ Unusual Severe Case of a Fire Eater's Lung}

J. Maxén, R. Bumm, M. Furrer, T.D. Latshang, P. Ludwig

Kantonsspital Graubuenden, Chur, Switzerland

Introduction: Fire eater's lung (FEL) is a form of acute chemical toxic pneumonitis, which is caused by accidental aspiration of flammable petrochemical derivates. The literature suggests a conservative therapeutic approach. Courses with severe complications with need of a surgical intervention are very rare.

Patient History: A 29-yr-old male was referred to our hospital because of severe pleuritic left-sided chest pain after accidental aspiration of Pyrofluid Safex $\odot$ while performing in a show as a fire eater. Morphine $6 \mathrm{mg}$ i.v. didn't relieve his pain sufficiently.

Initial Assessment and Therapeutic Interventions: After completing patient history and performing a computed tomography showing an extensive pulmonary consolidation in the left lower lobe we diagnosed an acute chemical toxic pneumonitis, also called fire eaters lung (FEL).

Clinical Course: Because of a lack of clinical improvement with conservative therapy, rising inflammatory parameters, suspected occlusion of the bronchus of the left lower lobe and increasing pleural effusion with multiple septae we performed a bronchoscopy and a drainage of the effusion. Bronchoscopy showed a high grade stenosis due to inflammation and edema of the mucosa. Bronchial lavage revealed staphylococcus aureus, whereas pleural fluid showed no bacterial growth. After a course of 7 days of antibiotics and still persistent therapy-resistant pain we performed a thoracoscopic debridement and decortication. Only after this this intervention a continuous clinical and radiological improvement was observed. The patient recovered completely after several weeks.

Conclusion: In contrast to other reports of FEL our case showed a very unusual severe course with complicated effusion as a consequence of the chemical toxic pneumonitis. Whereas most cases can 
be managed with a conservative approach, in our patient a thoracoscopic debridement was critical and provided a complete recovery.

We suspect that the outcome would have been worse without surgical intervention with persistent defect after healing.

\section{Poster Walk 3: Clinical Lung / Patient Management 2}

P22

When the Hummingbird Coughs

S. Guerin $^{1}$, G. Duvoisin' ${ }^{2}$, M. Koob ${ }^{3}$, A. Nydegger ${ }^{2}$, I. Rochat ${ }^{1}$

${ }^{1}$ Unité de Pneumologie Pédiatrique - Département Femme Mère Enfant, ${ }^{2}$ Unité de Gastroenterologie Pédiatrique Département Femme Mère Enfant, ${ }^{3}$ Service de Radiodiagnostic et Radiologie Interventionnelle, CHUV, Lausanne, Switzerland

Introduction: Chronic cough is a common symptom in the paediatric population. A careful history and clinical examination are necessary to determine its origin, especially to differentiate respiratory from extra-respiratory causes, including digestive causes.

Description: We report the case of a previously healthy 9-yearold girl who presented with a 3-months history of nocturnal dry cough inducing nightly vomiting. There was no fever but significant weight loss was reported as well as progressive dysphagia to solids, less pronounced with liquids. The cough did not respond to a trial of inhaled corticosteroids and bronchodilators, nor to antibiotics prescribed for a supposed atypical pneumonia based on CXR findings. Clinical examination was non-contributive. Because of persisting symptoms, chest CT scan was performed and showed ground-glass multifocal opacities with centrolobular micronodules, associated with a dilated oesophagus, highly suspicious for achalasia and secondary microaspirations. Bronchoalveolar lavage showed no infectious agent, and 7\% lipid-laden macrophages. Contrast imaging of the oesophagus showed esophageal dilatation associated with a "bird's beak" appearance of the lower oesophagus, suggesting achalasia. The oesophageal manometry confirmed the absence of peristalsis with increased tone of the lower oesophageal sphincter, a finding also consistent with achalasia. Oral feeds were replaced by nasogastric feeding with rapid resolution of the cough, until the realization of a peroral endoscopic myotomy (POEM). Post-intervention regular oral feeding was started successfully later on without the recurrence of coughing.

Conclusion: Achalasia is a rare motility disorder of the oesophagus mainly presenting with dysphagia but chronic respiratory symptoms such as cough are also reported. Cough can be due to chronic aspiration or tracheal compression by the dilated oesophagus. This case shows that a thorough history and clinical exam are essential for the investigation of chronic cough to rule out extra respiratory causes, which do not respond to usual respiratory treatment.

\section{P23 \\ Treatment of a Persisting Bronchopulmonary Fistula with Endobronchial Valves in a Patient with Necrotising Pneumonia}

J. Wani, S. Braun, M. Hofer, T. Hess

Pneumologie, Kantonsspital Winterthur, Winterthur, Switzerland

Introduction: Occlusion of the affected segment by endobronchial valves (EBV) is an accepted treatment option of a localized bronchopleural fistula with persistent air leak after pulmonary resection, in spontaneous pneumothorax and other perforating lung diseases.

Method: A 50-year-old man with chronic alcoholism was hospitalised because of a severe necrotising pneumonia of the right upper lobe (RUL) and middle lobe (ML) with empyema and spontaneous pneumothorax. Microbiology showed Streptococcus milleri in the pleural fluid and antibiotic treatment was initiated. A chest tube was inserted with good drainage of the empyema fluid but persistent air leak of up to $4 \mathrm{l} / \mathrm{min}$ over 3 weeks without tendency to decrease and incomplete expansion of the right lung. Surgery was denied because of the bad performance status and a bronchoscopic interventional therapy was performed instead.

Bronchoscopy with blockage of the RUL with a balloon catheter (Bronchusblocker ${ }^{\circledR}$ ) resulted in no decrease of the flow rate measured by Thopaz ${ }^{\circledR}$ drainage system. Simultaneous insertion of a second balloon into the ML resulted in immediate cessation of the air leak. During a second bronchoscopy with conscious sedation we placed 3 Zephyr ${ }^{\circledR}$ EBV-TS-4.0 and 1 Zephyr ${ }^{\circledR}$ EBV-TS4.0-LP into the 4 segmental bronchi of the RUL and 1 Zephyr ${ }^{\circledR}$ EBV-TS-5.5 into the ML.

Result: Immediately after EBV placement the initial air leak of $2-4 \mathrm{l} / \mathrm{min}$ decreased to $20 \mathrm{ml} / \mathrm{min}$ (supposed slight collateral ventilation) and ceased after a couple of days. Consequently the chest tube was removed. With prolonged antibiotic therapy over 8 weeks the patient showed good recovery. A problem-free extraction of the EBV was performed 3 month later.

Conclusion: For selected patients with persisting air leak because of necrotising pneumonia bronchial occlusion by multiple EBVs could be a therapeutic alternative to surgical resection after proof of sufficient blockade by (multiple) balloon catheter(s). 


\section{P24}

\section{Right Heart Catheterization in Takayasu's Arteritis: Do Not Occlude the Last Remaining Vessel!}

C. Teres $^{1,2}$, A.-L. Hachulla ${ }^{2,3,4}$, S. Noble ${ }^{1,2,5}$, F. Lador ${ }^{2,5,6}$

${ }^{1}$ Division of Cardiology, University Hospitals of Geneva, 2Pulmonary Hypertension Program, University Hospitals of Geneva, ${ }^{3}$ Division of Radiology, University Hospitals of Geneva, ${ }^{4}$ Faculty of Medicine, Geneva University, ${ }^{5}$ Faculty of Medicine, Université de Genève, ${ }^{6}$ Division of Pneumology, University Hospitals of Geneva, Geneva, Switzerland

Introduction: Takayasu arteritis is a large-vessel vasculitis that affects the aorta and its primary branches. The pathogenesis is not well understood and it can lead to narrowing and occlusion of the affected arteries causing heterogeneous symptoms. Observational studies have shown up to $50 \%$ of pulmonary circulation involvement.

Case Report: A 49-year-old woman known for a mild to moderate aortic regurgitation had a routine transthoracic echocardiography showing high probability of pulmonary hypertension (PHT).
Contrast enhanced CT depicted wall thickening of the aortic arch (Fig. 1c) and the main pulmonary artery with complete occlusion of the left and some segmental arteries of the right arterial tree (Fig. 1a). Dual-energy computed tomography (DECT) showed complete left and partial right pulmonary hypoperfusion (Fig. 1b) all in favor with large vessel vasculitis and Takayasu's arteritis (TA) was diagnosed.

Right and left heart catheterization revealed mild pre-capillary PHT (mPAP 26 mm Hg; PVR 3.4 WU). While wedging towards the right capillary circulation (7F Swan Ganz catheter, Fig. 1d), the patient presented dizziness and dyspnea and arterial blood oxygen saturation dropped to $69 \%$. Desaturation completely resolved after dewedging and deflating the balloon.

Discussion: This observation confirms the important involvement of the pulmonary circulation in this case of arteritis with the pulmonary perfusion relying on a last remaining permeable vessel despite a near normal resting pulmonary arterial pressure. A trial of immunosuppressive treatment (steroids and methotrexate) was started and the patient had a control catheterization at 8 months follow-up that showed haemodynamic normalization (mPAP 19 mm Hg, PVR 2.4 WU) but without reperfusion of occluded zones. A cardiopulmonary exercise test confirmed a preserved exercise capacity $(112 \mathrm{~W}, 22.4 \mathrm{ml} / \mathrm{kg} / \mathrm{min})$ with physiological cardiovascular and ventilator response despite increased physiological dead space.
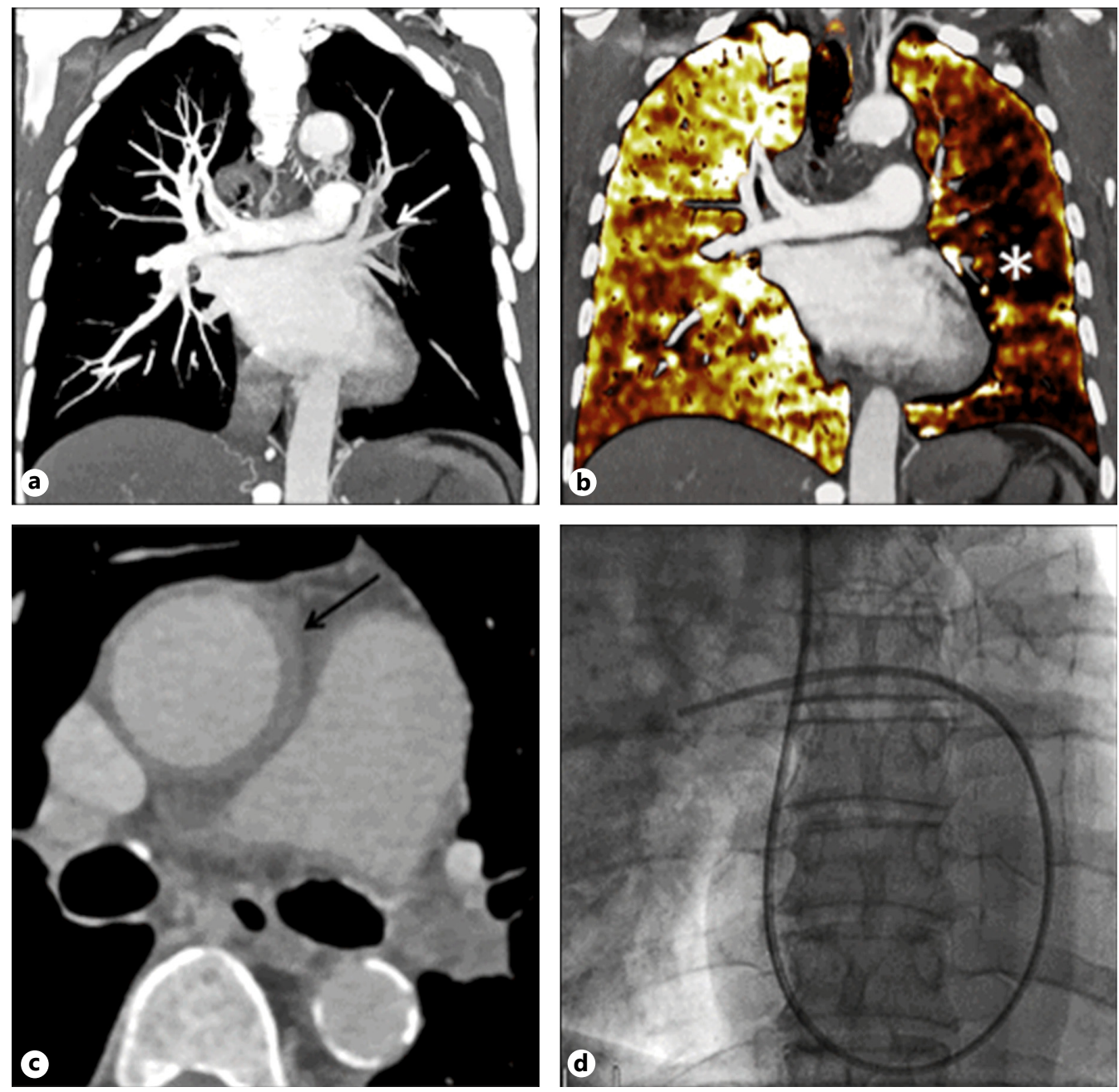

Fig. 1. (for Abstract P24). 


\section{P25 \\ Microwave Ablation in Managing Hemoptysis from Lung Cancer}

\author{
S. Saenghirunvattana ${ }^{1}$, C. Napairee ${ }^{2}$, S. Geraplangsub ${ }^{2}$, \\ P. Wetchanien ${ }^{2}$ \\ ${ }^{1}$ Bangkok Hospital Medical Center, Pulmonary and Chest Center, \\ ${ }^{2}$ Bangkok Hospital Medical Center, Bangkok Dusit Medical \\ Services, Bangkok, Thailand
}

Introduction: Patients diagnosed of advanced stage of lung cancer and inflammatory myofibroblastic tumor (IMT) who developed massive hemoptysis has limited options. The use of microwave ablation (MWA) is proven to be a helpful technique.

Methods: These are two case reports of advanced lung cancer and IMT utilizing MWA to manage hemoptysis and control tumor growth of patients that are unable to undergo complete lung resection.

\section{Results:}

Case Report No. 1: A 69-year-old female was diagnosed of lung adenocarcinoma, stage IV. Tyrokinase inhibitor (TKI) was provided for a year until her follow up chest CT scan reported primary lesion has increased from $2.7 \times 1.8 \mathrm{~cm}$ to $3.2 \times 2.0 \mathrm{~cm}$. Repeat biopsy was positive of adenocarcinoma. She refused to undergo chemo and radiation therapy and prefers only surgical management which was not advised due to the extent of disease. Later, she developed moderate hemoptysis. Microwave ablation as palliative therapy was offered as an option. Immediately post MWA, no more hemoptysis was noted.

Case Report No. 2: A 29-year-old male diagnosed of inflammatory myofibroblastic tumor (IMT) who is unresponsive to low dose corticosteroids and azathioprine and complains of progressive hemoptysis.

Chest CT scan revealed consolidative mass like lesion at the right upper lung, measuring 4.9x8.6 cm in size, with internal calcifications and mass extended into the right main bronchus, multiple spiculate nodules at both lungs are detected. MWA was performed on right upper lung mass. Bleeding was completely controlled. This is the first case of IMT of the lung reported to be destroyed by MWA and first reported case of using MWA with stent placement.

Conclusion: Microwave ablation therapy is a safe and effective tool for treatment of massive hemoptysis caused by lung cancer. This does not only help control tumor but this can also be used in complete termination of bleeding.

\section{P26 \\ Use of the Asthma-Tracker App: Cross-Sectional Data Analysis}

F. Bierreth $^{1,2}$, A. Naduvilekoot ${ }^{1,2}$, T. Dieterle ${ }^{1,2}$, J.D. Leuppi ${ }^{1,2}$

${ }^{1}$ Cantonal Hospital Baselland, Liestal, ${ }^{2}$ Faculty of Medicine, University of Basel, Basel, Switzerland

Introduction: Asthma bronchiale represents a major global health burden. However, asthma control is still not satisfactory. Therefore we developed the Asthma-tracker-App, which can easily be integrated into everyday routine. The aim of this cross-sectional data analysis is to provide an overview of the use of the Asthma-app. Detailed information can be found under: "www.asthmaapp.ch".

Methods: The Asthma-app was designed and developed for patients with asthma bronchiale and can be used as a diary for the recording of different parameters like peak-flow values, asthma symptoms, use of inhaled medication and completion of the asthma control test (ACT) once weekly. Moreover, the app is able to give reminders for measuring peak expiratory flow (PEF).

Results: The presented data show the use of the Asthma-app for December 2017. In total, the app was used by 469 users with 51814 records. It was used on a mean of 5.78 days per month, mostly for entering information about PEF (42.18\%) and information regarding their inhaled medication (43.42\%). The functions for recording symptoms $(7.74 \%)$ as well as for the completion of the ACT questionnaire (6.66\%) were used rarely. Figure 1 shows the mean records during the weekdays. The most records were observed on Saturday and Sunday while the lowest use was on Monday and increased till Saturday with a decrease on Friday.

Conclusion: The app was mostly used on Saturdays and Sundays, showing a preference to enter the data on weekends. It also appears that the users prefer to enter information about their PEF and their inhaled medication rather than their symptoms or ACT.

\section{P27}

\section{Bronchoscopy Under Treadmill Test Detects Instability of the Right Main Bronchus after Extended Extrapleural Lobectomy of the Right Upper Lobe}

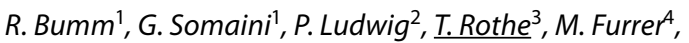 \\ T.D. Latshang ${ }^{2}$ \\ ${ }^{1}$ Thoracic Surgery, ${ }^{2}$ Pneumology, Cantonal Hospital Chur, \\ ${ }^{3}$ Departement Innere Medizin - Pneumologie, Kantonsspital \\ Graubuenden, ${ }^{4}$ Cantonal Hospital Chur, Chur, Switzerland
}

Introduction: Dyspnoea due to instability of the main bronchus after extended upper lobe resection is a very uncommon finding. Here, one particular case is presented focusing on the postop- 


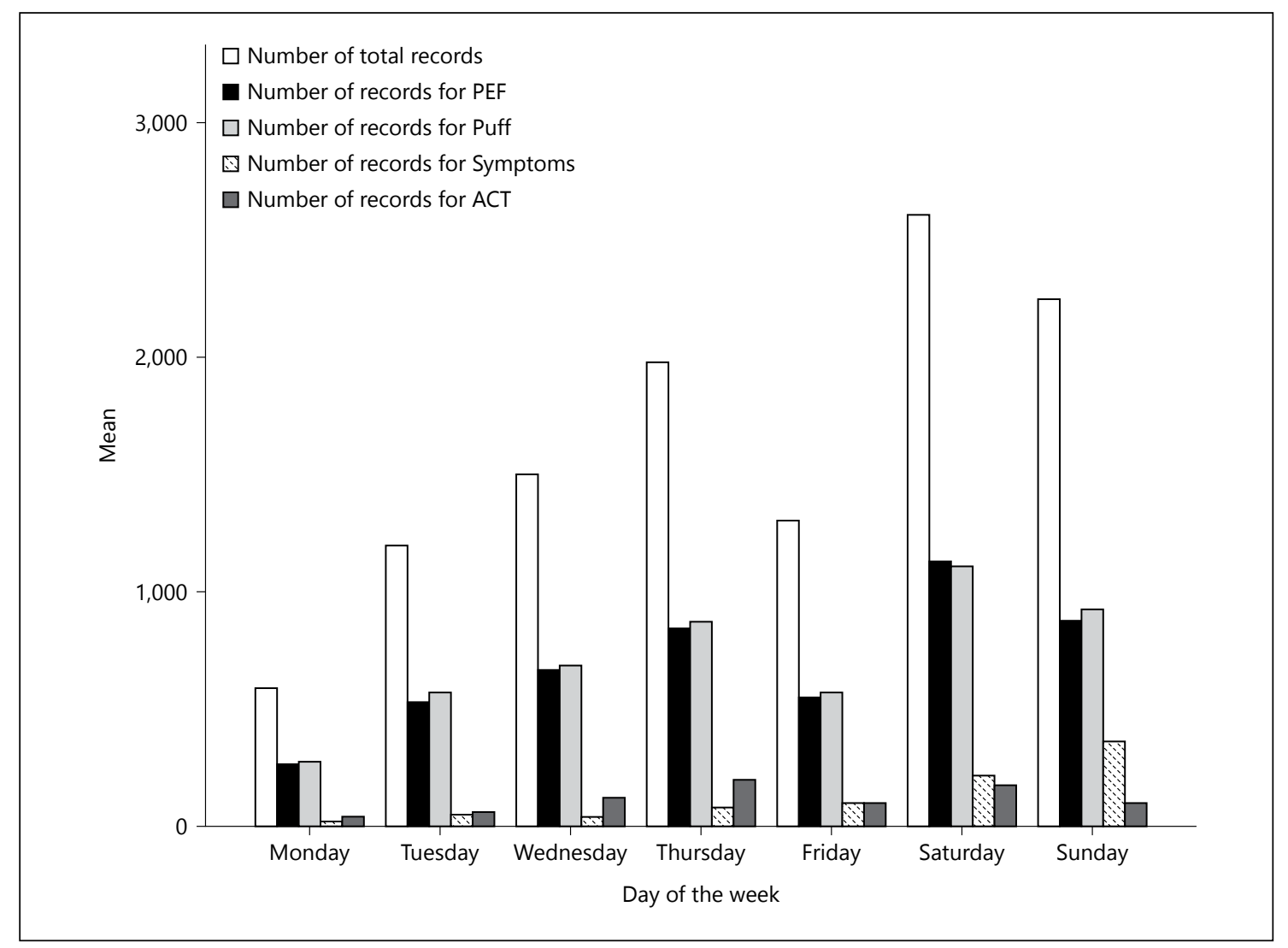

Fig. 1. Mean numbers of records for the investigated month (for Abstract P26).

erative diagnostic work-up by cine-MRI and flexible video-bronchoscopy while performing a treadmill test.

Method: A 44 year old male patient was submitted with a bulky pleural tumour being related to the right upper lobe and the chest wall. A soft tissue sarcoma was suspected and the patient underwent an extended open and extrapleural lobectomy of the right upper lobe with chest wall resection. Histology revealed a giant intrapleural leiomyoma. The postoperative course was uneventful. However, 6 weeks later the patient presented with progressive dyspnoea and chest pain. A conventional work-up did not clarify the situation. The patient underwent lung function testing that revealed a combined severe restrictive and obstructive pattern. In order to further evaluate the functional deficit, the patient was scheduled for a cine-MRI scan as well as a flexible bronchoscopy while performing a treadmill test.

Results: Dynamic MRI scan did not detect instability of the chest wall or herniation of the lung. However, bronchoscopy, when performing $100 \mathrm{Watt}$ in the treadmill test, showed a progressive functional stenosis of the right main bronchus during inspiration with related collapse of the lateral bronchial wall at the tracheobronchial angle. In addition, an expiratory protrusion of the pars membranacea of the trachea into the right main bronchus became obvious. The functional disorder had an inspiratory and expiratory component.
Conclusion: Flexible bronchoscopy under physical effort was able to demonstrate a clinically significant airway obstruction that occurred after uneventful extended lobectomy of the right upper lobe. The patient is under consideration for rethoracotomy and sleeve resection or airway-stenting of the respective bronchial segment.

P28

Severe Asthma in Switzerland: Overview of the Population and Treatments by Specialists

\section{P. Jandus $^{1}$, M. Jacobshagen ${ }^{2}$, A. Longatti ${ }^{2}$, P. Bonvin ${ }^{2}$}

'Service d'Allergologie et d'Immunologie, Hôpitaux Universitaires de Genève, Genève, ${ }^{2}$ Novartis Pharma Schweiz A.G., Rotkreuz, Switzerland

Introduction: Although the majority of asthma patients can be effectively treated with currently available medications, $5-10 \%$ of them develop a severe asthma. No recent national data are currently available, we therefore developed a survey to investigate 
how severe asthma is currently diagnosed and treated in Switzerland.

Methods: The survey assessed the habits and knowledge of Swiss specialists regarding the diagnosis, evaluation and treatment of their severe asthmatic patients. The survey consisted of 19 questions and was distributed to Swiss pulmonologists and allergists from $01 / 2017$ to $01 / 2018$.

Results: 23 specialists answered to the survey. $52 \%$ of the physicians felt very confident in diagnosing severe asthma. Overall, they estimated that $51 \%$ of their asthmatic patients have a respiratory allergy and that $28 \%$ of them are polymorbid. The most frequently mentioned comorbidities were cardiovascular diseases (59\%) and psychological disorders (35\%). Inhaled corticosteroid/ long-acting beta-agonist combinations, leukotriene receptor antagonists and monoclonal antibodies (mAbs) were considered by more than $80 \%$ of specialists as potential background therapy whereas oral corticosteroids (OCS) were mentioned by $74 \%$ of them. Although no long-acting muscarinic antagonist is currently approved for the treatment of asthma in Switzerland, it was considered as a potential treatment by more than half of the physicians. 57\% of physicians reported using OCS before mAbs in their severe asthmatic patients, which may be linked to the fact that the majority (65\%) reported limited experience with the clinical use of biologics.

Conclusion: These results support the idea that diagnosis and treatment of severe asthma are not obvious, even in a resourceful healthcare system. In contradiction with the GINA guidelines, the majority of Swiss specialists still mentioned OCS before mAbs in their own asthma treatment algorithm. Further, this survey highlights the need of more studies regarding management of polymorbid severe asthma patients which consist a quarter of the population.

\section{P29 \\ Chylothorax in Lymphangioleiomyomatosis: Efficacy of Sirolimus}

\author{
E. Diamanti ${ }^{1}$, C. Daccord ${ }^{1}$, I. Skaventzos ${ }^{2}$, R. Lazor ${ }^{1}$ \\ ${ }^{1}$ Respiratory Medicine Departement, CHUV, Lausanne, \\ ${ }^{2}$ General Medicine Practice and Pneumology, Chateau-d'Oex, \\ Switzerland
}

Introduction: Lymphangioleiomyomatosis (LAM) is a rare disease affecting young women, and characterized by multiple lung cysts, recurrent pneumothorax, chylous effusions, and renal angiomyolipomas. LAM is caused by the proliferation and dissemination of low-grade tumoral cells (LAM cells) bearing inactivating mutations in the TSC2 gene, with consequent loss of function of tuberin, an inhibitor of the mammalian/mechanistic target of rapamycin (mTOR), which plays a central role in cell growth regulation.

Case Presentation: A previously healthy 46-year old woman presented with dyspnea NYHA class II-III, non-productive cough, minor hemoptysis, left chest pain and diminished breath sounds on the left lung base. Chest computed tomography revealed multiple lung cysts and a large left pleural effusion. Thoracentesis showed a "milky" exudate with high triglycerides concentration, diagnosed as chylothorax. Abdominal CT identified a left renal tumor of $13 \mathrm{~mm}$ diagnosed as angiomyolipoma. Vascular endothelial growth factor D was $655 \mathrm{pg} / \mathrm{ml}$ (diagnostic threshold for LAM: $>800 \mathrm{pg} / \mathrm{ml}$ ). There were no features of tuberous sclerosis, and sporadic LAM was diagnosed. The chylothorax requested thoracentesis every 10-15 days for several months to reduce dyspnea. Based on recent guidelines recommending mTOR inhibitors as first-line therapy for chylothorax in LAM, sirolimus was initiated at $2 \mathrm{mg} /$ day. Over the subsequent 3 months, the volume of chyle removed at each thoracentesis, and the size of pleural effusion on consecutive chest X-rays progressively decreased. Simultaneously, forced vital capacity markedly improved from $2.74 \mathrm{~L}$ (70\%pred) to $3.73 \mathrm{~L}$ (95\%pred). 6 months after sirolimus initiation, chest X-ray showed no effusion and remained normal for another 6 months with continued therapy.

Conclusion: In agreement with previous observations, sirolimus led to complete and sustained resolution of chylothorax in our patient. mTOR inhibitors are now the first-line therapy in LAMassociated chylous effusions. Pleurodesis and thoracic duct ligation are only second-line treatments.

\section{P30 \\ Lung Laceration as a Rare Complication after Endobronchial Valve Implantation}

S. Filippi

Pneumologie, Departement Medizin, Kantonsspital Winterthur, Winterthur, Switzerland

Introduction: Endobronchial valve (EBV) placement is a wellestablished procedure in severe emphysema with marked hyperinflation without collateral ventilation.

We present a rare complication of lung laceration after EBV placement.

Case Report: A 69-year-old male patient with severe COPD GOLD IV and severe hyperinflation was referred because of persistent dyspnea.

The medical history is remarkable for a right-sided spontaneous pneumothorax treated by surgical lung volume reduction and talc pleurodesis without functional benefit. After exclusion of collateral ventilation by Chartis measurement we performed a bronchoscopic insertion of $6 \mathrm{EBV}$ (Zephyr) in the left upper lobe.

After the procedure the patient reported a significant improvement. Chest x-ray showed atelectasis of the left upper lobe with marked elevation of the left diaphragm. On the $5^{\text {th }}$ day he developed a left sided pneumothorax. Drainage was inserted with incomplete expansion and persistent bronchopleural fistula. A CT-scan demonstrated an encapsulated fluid-air collection adjacent to the oblique fissure (Figure 1). After extraction of the valve in LB5 the air leak disappeared, and the drainage could be removed. Three weeks later he returned with fever, elevated CRP and persistent left sided fluid-air collection. A pigtail catheter was inserted and hematoma infected by Eikenella corrodens drained. After removal of all EBVs thoracoscopy showed a laceration of the lung near the oblique fissure. A resection of the left apical lower lobe was performed. 
Result: 5 weeks after resection of the left apical lower lobe the patient reported a relevant amelioration of his performance. FEV1 improved by $340 \mathrm{ml}(960$ to $1130 \mathrm{ml}), 6-\mathrm{MW}$-distance by $90 \mathrm{~m}$ (360 to $450 \mathrm{~m}$ ).

Conclusion: This case demonstrates a lung laceration after EBV insertion presumably provoked by a large shift of the left lower lobe. After combined surgical resection and lung volume reduction the patient showed a marked improvement of lung function and 6-MW-distance.

\section{P31 \\ Conversion of Quantiferon-TB Gold Plus Following Isoniazid Prophylaxis among Latent Tuberculosis Patients}

\section{S. Saenghirunvattana, T. Kaenpon}

Pulmonary and Chest Center, Bangkok Hospital Medical Center, Bangkok Dusit Medical Services, Bangkok, Thailand

Introduction: Tuberculosis (TB) remains to be one of the most common infectious diseases worldwide. World Health Organization global TB report in Thailand noted 62,135 new and relapse cases in 2015. Treatment of latent TB infection is an important factor in TB elimination plan. Diagnosis and medication evaluation through biomarkers are crucial factors for better control of spread of infection.

In 2011, Komiya et al reported a fifty-eight percent conversion rate of active tuberculosis patients from positive QuantiFERONTB Gold to negative results five to seven months post-treatment. In this study, we assessed the use of QuantiFERON-TB Gold Plus in diagnosis of latent TB and response to a nine-month Isoniazid prophylaxis.

Methods: We conducted a case series on December 2016 and November 2017. Twenty asymptomatic cases were identified to have had exposure to active TB infection, undergone chest $\mathrm{x}$-ray and QuantiFERON-TB Gold Plus test. Those with positive QuantiFERON-TB Gold Plus result with negative chest $x$ ray report were prescribed with Isoniazid $300 \mathrm{mg}$ tablet once daily for nine months. Post prophylaxis, chest-x ray and QuantiFERON-TB Gold Plus test were repeated to determine conversion results.

Results: Among the twenty participants, all were positive of QuantiFERON-TB Gold Plus test and negative of chest X-ray. The average age was $47 \pm 5.7$ years and there were eighteen females and two males. Two had underlying conditions: valvular heart disease and peripheral neuropathy. All took the nine-month Isoniazid prophylaxis and repeated QuantiFERON-TB Gold Plus test and chest $\mathrm{X}$-ray. Chest X-ray results again were all negative. Eighteen cases revealed persistence of QuantiFERON-TB Gold Plus but two cases were negative. The conversion rate of QuantiFERON-TB Gold Plus was ten percent.

Conclusion: Our series revealed the first report of ten percent conversion rate of QuantiFERON-TB Gold Plus among latent TB participants who were prescribed with a nine-month Isoniazid prophylaxis.

\section{P32}

Bilateral Phrenic Nerve Paresis - But Still Enough Air

\section{G. Hässig ${ }^{1}$, K. Huss-Mischler ${ }^{2}$, T. Latshang ${ }^{3}$, M. Furrer ${ }^{4}$}

${ }^{1}$ Thoracic and Vascular surgical Unit, Kantonsspital Graubuenden, ${ }^{2}$ Oncology, ${ }^{3}$ Pneumology, ${ }^{4}$ Thoracic and Vascular surgical Unit, Chur, Switzerland

In case of progressive speech dyspnea and shortness of breath while lying down, we primarily consider a pulmonary or cardiac problem. In this case-report we demonstrate an impressive differential diagnosis of a patient with a huge mediastinal tumour and bilateral phrenic nerve palsy. This clinical constellation outside of neurological diseases is rarely documented in the literature.

A 56-year-old smoker and radio-presenter, introduces himself to his general practitioner to clarify an increasing speech dyspnea. Conventional radiology reveals an impressive tumour in the thorax with moderate restriction of lungfunction.

In the Computer Tomography with needle puncture the diagnosis of a neuroendocrine tumour is made. After the usual interdisciplinary preoperative investigations, tumour resection was performed using a clamshell approach extended by an additional median sternotomy. The pericardium and the N. phrenicus had to be resected on both sides to achieve a radical tumour resection. Postoperatively, the patient was able to compensate surprisingly well the lack of diaphragm innervation by using the accessory muscles of respiration, so that pre-existing phrenic nerve paresis was suspected due to tumour invasion. Six months after surgery, an improved lung function with only borderline restriction and a normal ergospirometry was found and no tumour recurrence was obvious. Nighttime hypoventilation had already disappeared completely three months after surgery.

In the definitive histological result an atypical carcinoid of the thymus was found. A relationship with a carcinoid associated MEN type 1, Hippel Lindau syndrome, neurofibromatosis or tuberous sclerosis could not be found.

Whether the preoperative shortness of breath was caused by the infiltrated and therefore non-functional Nn. phrenici or by the size of the tumourmass alone cannot be conclusively determined. The fact that the patient is practically free of symptoms in the midterm course underlines the possibility of bilateral nerve resection when dictated by radicality in such difficult decision finding situations. 


\section{Poster Walk 4: Sleep / Ventilation / Respiratory Technology \& Experimental/Bench \& Occupational Respiratory Disease}

\author{
P33 \\ The Effects of CPAP-Withdrawal on Cerebral \\ Vascular Reactivity and Brain Oxygenation \\ in Patients with Obstructive Sleep Apnoea: \\ A Randomised-Controlled Trial
}

S. Thiel' ${ }^{1}$, F. Lettau' ${ }^{1}$, S.H. Haile ${ }^{2}$, P. Rejmer ${ }^{1}$, N. Sievi ${ }^{1}$, E.I. Schwarz' ${ }^{1}$, A. Stöberl' ${ }^{1}$, C. Rossi ${ }^{3}$, A. Boss ${ }^{3}$, A. Becker ${ }^{3}$, J.R. Stradling ${ }^{4}$, M. Kohler ${ }^{1,5}$

${ }^{1}$ Sleep Disorders Centre and Pulmonary Division, University Hospital Zurich, ${ }^{2}$ Epidemiology, Biostatistics and Prevention Institute, University of Zurich, ${ }^{3}$ Department for Radiology, University Hospital Zurich, Zurich, Switzerland, ${ }^{4}$ Oxford Biomedical Research Centre based at Oxford University Hospitals NHS Foundation Trust and University of Oxford, Oxford United Kingdom, National Institute for Health Reserch (NIHR), Oxford, United Kingdom, ${ }^{5}$ Centrer for Integrative Human Physiology, University of Zurich, Zurich, Switzerland

Introduction: Disturbed cerebral perfusion due to impaired cerebral vascular reactivity (CVR) has been shown to elevate longterm stroke risk. Obstructive sleep apnoea (OSA) is associated with vascular dysfunction and cardiovascular events. The aim of this study was to evaluate the effect of continuous positive airway pressure (CPAP) withdrawal on CVR and brain oxygenation.

Methods: Patients with moderate-to-severe OSA effectively treated with CPAP were randomised to continue or withdraw CPAP for two weeks. At baseline and after two weeks patients underwent a sleep study and magnetic resonance imaging (MRI). CVR was quantified using blood oxygen level-dependent (BOLD)-MRI during hyperoxic and hypercapnic stimuli. The treatment effect on CVR was determined by regression analysis.
Results: 43 subjects ( $81 \%$ male, mean age $57 \pm 9.9$ yrs) participated. OSA did recur in the sham-CPAP (mean [SD] change in AHI of 35.1 [29.6]) but remained controlled in the CPAP group. CPAP withdrawal was associated with a reduction on grey matter BOLD Signal (\%) during hyperoxic stimulus (mean withdrawal effect $(95 \%$ CI $)-0.53(-1.09 / 0.30) \%, p=0.06)$ while hypercapnia showed no significant signal change. Whole brain BOLD Signal (\%) did not change under both, hyperoxic and hypercapnic, conditions (Table 1).

Conclusion: Preliminary data analysis suggests an impaired CVR in grey matter in patients with OSA during CPAP-withdrawal. Implications on cardiovascular risk remains to be clarified.

\section{P34 \\ Risk Factors for Unintentional Leaks during Home Non-Invasive Ventilation}

D. Adler ${ }^{1}$, C. Cantero ${ }^{1}$, P. Pasquina ${ }^{1}$, A.B. Younossian ${ }^{2}$, M. Prella ${ }^{3}$, P.M. Soccal ${ }^{1}$, J.-P. Janssens ${ }^{1}$

${ }^{1}$ Service de Pneumologie, HUG, ${ }^{2}$ Service de Pneumologie, Hôpital de la Tour, Genève, ${ }^{3}$ Service de Pneumologie, CHUV, Lausanne, Switzerland

Background: Intentional air leaks through a vented mask are inherent to home NIV (HMV) and necessary for $\mathrm{CO}_{2}$ removal. Conversely, unintentional air leaks can interfere with treatment efficacy by reducing upper airway patency, decreasing alveolar ventilation and affecting trigger function. Therefore, reducing leaks is an important treatment goal.

Aim: To investigate modifiable and non-modifiable risk factors associated with unintentional leaks during HMV.

Methods: This study is part of a cross-sectional multicentric study of patients undergoing long term ( $>3$ months) HMV in the Cantons of Geneva and Vaud between 1.06.2016 and 31.06.2017. Patients treated with adaptive servo-ventilation or with ventilators not reporting unintentional leaks were excluded from this analysis. $95^{\text {th }}$ percentile of unintentional leaks $(\mathrm{L} / \mathrm{min})$ over a 30-day period prior to medical consultation was recorded at stable state during follow-up. Linear regression models were used to assess the association between unintentional leaks and risk factors.

Table 1. (for Abstract P33)

\begin{tabular}{|c|c|c|c|c|c|c|c|c|c|}
\hline & & $\begin{array}{l}\text { Sham group } \\
\mathrm{N}=20 \\
\text { Baseline }\end{array}$ & $\begin{array}{l}\text { Sham group } \\
\mathrm{N}=20 \\
\text { follow-up }\end{array}$ & Change & $\begin{array}{l}\text { CPAP group } \\
\mathrm{N}=20 \\
\text { Baseline }\end{array}$ & $\begin{array}{l}\text { CPAP group } \\
\mathrm{N}=20 \\
\text { Follow-up }\end{array}$ & Change & Treatment effect* & $\begin{array}{l}\mathrm{p} \text { value } \\
\text { adj. }\end{array}$ \\
\hline BOLD signal change grey matter & $\mathrm{CO} 2$ & $3.65(1.22)$ & $3.51(1.23)$ & $-0.14(1.10)$ & $3.88(2.01)$ & $3.52(1.12)$ & $-0.36(1.95)$ & $-0.05(-0.64 / 0.75)$ & 0.873 \\
\hline BOLD signal change overall & $\mathrm{O} 2$ & $2.34(0.83)$ & $2.51(0.92)$ & $0.18(0.67)$ & $2.75(1.53)$ & $2.72(0.50)$ & $-0.03(1.56)$ & $-0.12(-0.58 / 0.33)$ & 0.586 \\
\hline BOLD signal change overall & $\mathrm{CO} 2$ & $3.00(1.00)$ & $3.23(1.01)$ & $0.23(0.96)$ & $3.18(1.14)$ & $3.15(0.70)$ & $-0.03(1.23)$ & $0.13(-0.39 / 0.66)$ & 0.609 \\
\hline
\end{tabular}

Values are presented as mean (SD) or 95\% CI. * adj. for Baseline of sham. 
Results: 327 patients (56\% men, median age 71 yrs, IQR: $59-$ 77) treated with HMV were included: 96 (30\%) COPD; 35 (11\%) overlap syndromes; 81 (25\%) OHS; 28 (9\%) OSA; 78 (25\%) NMD; diagnosis was missing for 9 patients. For the whole group, unintentional leaks were $14 \mathrm{~L} / \mathrm{min}$ (IQR: 2.4-30). Univariate regression analysis demonstrated an association between unintentional leaks and age (coef 0.32, SE 0.12, p = 0.007), full face mask (coef -6.09 , SE 3.93, $\mathrm{p}=0.123$ ), IPAP (coef 0.97, SE 0.43, $\mathrm{p}=0.023$ ), EPAP (coef 1.24 , SE $0.54, \mathrm{p}=0.024$ ) but not with gender or time since initiation of HMV. Using backward stepwise selection in a multivariate model incorporating all variables described above, only age (coef 0.38 , SE 0.12, p = 0.002), full face mask (coef -9.95 , SE 3.96, p = 0.012 ) and IPAP (coef 1.14, SE 0.43, p = 0.008) remained associated with unintentional leaks.

Conclusions: Older age, use of nasal prongs or nasal mask, and higher inspiratory pressure are independently associated with unintentional leaks during HMV.

\section{P35 \\ Thoracoscopic Thymectomy during Myasthenic Crisis in a Patient with Late Onset Myasthenia Gravis Associated with Thymoma}

G. Somaini ${ }^{1}$, C. Michelitsch ${ }^{1}$, R. Sturzenegger ${ }^{2}$, P. Vanek ${ }^{3}$, M. Furrer ${ }^{1}$

${ }^{1}$ Department of Surgery, ${ }^{2}$ Department of Neurology,

${ }^{3}$ Department of Intensive Care Medicine, Kantonsspital

Graubuenden, Chur, Switzerland

Objective: Thymectomy is indicated in all patients with thymoma-associated myasthenia gravis (MG). But thymectomy has not been recommended during myasthenic crisis and is known to be a risk factor for postoperative worsening. Steroids are the most effective treatment for MG, but may initially cause disease exacerbation.

Methods: A 51-year-old man was referred with rapidly progressive weakness of shoulder and face muscles. A thymoma was radiologically seen on CT. The diagnosis of a late onset MG was made and oral corticosteroids and cholinesterase inhibitors were initiated. After initiation of therapy deterioration occurred due to myasthenic crisis. The patient presented with massive dyspnea and respiratory insufficiency, resulting in intubation, mechanical ventilation, and early tracheotomy has been performed in the intensive care unit.

There was no clinical improvement despite administration of intravenous immunoglobulin. After unsuccessful extubation, we decided to perform robot-assisted thoracoscopic (RATS) extended thymectomy by a right thoracic approach.

Results: The surgery was performed without complications. The patient recovered well from the surgery and was rapidly extubated and transferred to a rehabilitation facility. His MG symptoms improved allowing to rapidly tapering the oral medication.

Conclusion: If medical treatment is unable to stabilize the patient's condition during a myasthenic crisis minimal invasive surgery can be a straightforward and successful approach in these patients.

\section{P36 \\ How to Manage Tracheal Stenosis in a Patient with Tetraplegia. Case Report and Review of the Literature}

A. Merkel ${ }^{1}$, F. Michel ${ }^{2}$, M. Tamm ${ }^{3}$

${ }^{1}$ Klinik für Neurorehabilitation und Paraplegiologie, ${ }^{2}$ Rehab

Basel, ${ }^{3}$ Pneumologie, Universitätsspital Basel, Basel, Switzerland

A female patient 54 years old with complete tetraplegia C5, ASIA A, since a motorbike accident 1983 suffers from bronchitis and broncho-pneumonia due to retention of mucus with the need for repetitive hospitalisations. She uses CPAP during the night since the detection of an obstructive sleep-apnea syndrome 7/2015. Lung function evaluation revealed a severe neurogenic restrictive pattern in combination with typical signs of an extrathoracal stenosis. A high grade proximal circular stenosis due to long standing scars after tracheotomy and corrections of the tracheostoma during her first rehabilitation 1983 was bronchoscopically confirmed. CT-scan showed a goitre additionally compressing the trachea. During the short follow-up period after the evaluation the patient suffered from increasing dyspnea and had to be hospitalized due to acute ventilatory insufficiency. After a multidisciplinary team approach the decision for strumectomy was made and carried out followed by a short recovery period without the need for tracheotomy and a favorable course as well clinically as in lung function. Therapeutic options for para- and tetraplegic patients with tracheal narrowing will be discussed.

\section{P37 \\ Supportive Interaction of PI3K-Akt-mTOR and STAT3-miR21-PTEN Controls IgE Induced Airway Remodeling}

\section{L.Fang, M. Tamm, D. Stolz, M. Roth}

University Hospital Basel, Basel, Switzerland

Introduction: Irreversible airway remodeling in allergic asthma is often linked to increased serum IgE and features with airway smooth muscle cells (ASMC) hypertrophy and/or hyperplasia. ASMC express IgE receptors and respond to IgE by increased extracellular matrix (ECM) production and proliferation. This study investigates the mechanism of IgE induced ASMC remodeling, focusing on intracellular signaling transduction and epigenetic regulation.

Methods: Human ASMC were isolated and expanded from bronchial endoscopic biopsies of asthma patients $(n=15)$. ASMC were stimulated with human non-antigenic IgE $(1 \mu \mathrm{g} / \mathrm{ml})$ for 48 hours. Protein phosphorylation was detected by western blot, and selective signaling inhibitors were used to test signaling pathways. The production ECM was evaluated by cell based ELISA. Mitochondria mass and activity were determined by confocal microscopy for cytochrome $c$, and by real-time PCR for PGC1 $\alpha$ and PPAR $\gamma$, as well as for microRNA21-5p expression. The effect of 
microRNA21-5p on signaling pathways was assessed by specific mimics or inhibitors overexpression.

Results: IgE increased the phosphorylation of both STAT3, p70S6 kinase, and the transcription of microRNA21-5p. MicroRNA21-5p expression reduced PTEN expression, while overexpression of microRNA21-5p mimics or inhibitors altered PTEN expression accordingly. PI3K, mTOR and STAT3 inhibitors blocked IgE induced ASMC remodeling. IgE significantly increased mitochondrial mass, gene expressions, and collagen-type-I and fibronectin deposition.

Conclusion: IgE promoted ASMC remodeling through two parallel signaling pathways, which merge at the level of mTOR and subsequently up-regulated mitochondrial activity and ECM production.

\section{P38}

\section{Cell Type Specific Heat-Shock-Protein Responses to Bronchial Thermoplasty in Severe Asthma Treatment}

\section{Fang, M. Tamm, M. Roth, D. Stolz}

University Hospital Basel, Basel, Switzerland

Background: Bronchial thermoplasty (BT) delivers heats $\left(65^{\circ} \mathrm{C}\right)$ to airway tissue by radiofrequency, and is the only therapy that improves life quality for patients with uncontrolled severe asthma. BT reduces sub-epithelial mesenchymal cell numbers and induces re-epithelization in airway. Although assumed to result from 'burning out smooth muscle', this hypothesis is insufficient to explain the lasting beneficial effects. This study assesses cell type specific effects of experimental BT on heat shock protein expression in primary human airway epithelial and sub-epithelial mesenchymal cells.

Methods: Human airway epithelial (AEC) and smooth muscle cells (ASMC) were isolated and expanded from endoscopic bronchial biopsies of severe asthma patients. Cells characterization: AEC stained positive for E-cadherin and cytokeratin, while ASMC positive for fibronectin and $\alpha$-smooth muscle actin ( $\alpha$-SMA) by immunohistochemistry. BT was mimicked by submerging subconfluent cells into a $65^{\circ} \mathrm{C}$ water bath for 10 seconds, and afterwards were grown under standard conditions for up to 48 hours. Cell proliferation was determined by manual cell counts. Heat stress response was evaluated by western-blot and immune-fluorescence microscopy for heat shock proteins HSP40, HSP60, HSP70, and HSP90.

Results: Experimental BT significantly increased AEC proliferation, while ASMC proliferation was interrupted. Western blot and immunofluorescences microscopy demonstrated a significant increase of HSP40, HSP60, HSP70 and HSP90 expression in AEC after $24 \mathrm{~h}$ and $48 \mathrm{~h}$. In contrast, no significant HSP up-regulation was observed in ASMC.

Conclusions: Cell type specific HSP response in AEC but not in ASMC indicates diversed survival fate of AEC over ASMC after $\mathrm{BT}$, by which mechanism that BT achieves its beneficial long-term effects in patients with severe asthma.

\title{
P39 \\ Dyspnea Interferes with Cognitive Functions: Protocol of a Randomized Trial
}

\author{
D. Lawi ${ }^{1}$, G. Berra' , G. Allali' ${ }^{2,3}$, E. Dupuis-Lozeron ${ }^{4}$, D. Adler ${ }^{1,3}$ \\ 'Service de Pneumologie, ${ }^{2}$ Service de Neurologie, Hôpitaux \\ Universitaires de Genève, ${ }^{3}$ Université de Genève, Faculté \\ de Médecine, ${ }^{4}$ Service d'Épidémiologie Clinique, Hôpitaux \\ Universitaires de Genève, Geneva, Switzerland
}

Background: Cognitive impairment is associated with decreased quality of life and increased mortality, but is underappreciated and poorly studied in chronic respiratory disorders. Normal breathing doesn't involve conscious perception. However, when breathing becomes difficult (i.e. when an inspiratory threshold is applied and give rise to respiratory-related motor cortical activity), "irruption of breathing into consciousness" may interfere with cognitive functions.

Like breathing, gait control is considered automatic. However, under certain conditions, such as in elderly subjects or patients with neuropsychiatric conditions, gait control becomes dependent

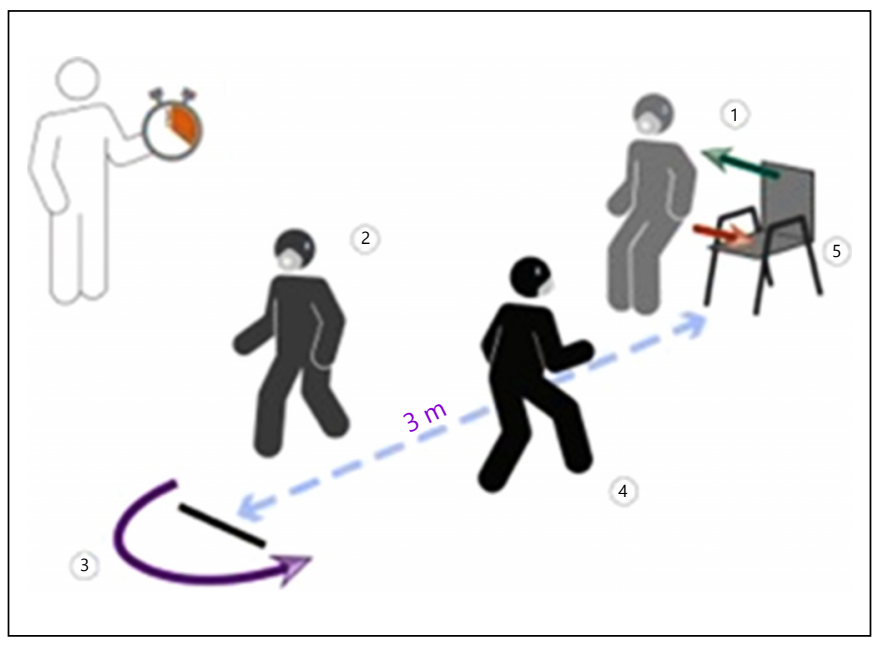

Fig. 1. Time up and go test (TUG). (1) On request, the participant gets up from the chair, (2) walks $3 \mathrm{~m}$, (3) turns around, (4) returns to his chair, and (5) sits back on the chair (for Abstract P39).

Table 1. (for Abstract P39)

\section{Participants characteristics}

Age, mean (range), y

Male, No (\%)

BMI $\left(\mathrm{kg} / \mathrm{m}^{2}\right)$

FEV1, mean, L

FEV1, mean, \% of predicted

VAS Dyspnea

Calm breathing period, units, max. 10

Dyspnea period, units, max. 10
$29(25-32)$

80

24

4.58

103

0

6.8 
Table 2. (for Abstract P39)

\begin{tabular}{lcccc}
\hline Outcome (Groups Combined (n=10)) & $\begin{array}{l}\text { Calm } \\
\text { breathing }\end{array}$ & Dyspnea & $\begin{array}{l}\text { Difference } \\
(95 \% \mathrm{CI})\end{array}$ & P value \\
\hline Time at Time Up ang Go Test (TUG), mean (sec.) & 8.6 & 9.3 & $0.7(0.29-1.11)$ & 0.0038 \\
Verbal fluency: Categorical, mean (words) & 33.5 & 30.3 & $3.2(4-10.5)$ & 0.34 \\
Verbal fluency: Lexical, mean (words) & 24.2 & 24.9 & $0.7(-5-6.7)$ & 0.72 \\
Trail Making Test A, mean (sec.) & 15.8 & 15.6 & $0.14(-2.2-2.5)$ & 0.89 \\
Trail Making Test B, mean (sec.) & 31.5 & 35.9 & $44.3(-12.6-3.9)$ & 0.26 \\
Test Code, mean (symbols) & 89.4 & 90.3 & $0.9(-12-11)$ & 0.86 \\
Memory Span: Direct mean (sequences) & 10.3 & 10.2 & $0.1(-1-1.2)$ & 0.84 \\
Memory Span: Indirect mean (sequences) & 6.1 & 7.6 & $1.5(-2.9-1)$ & 0.038 \\
\hline
\end{tabular}

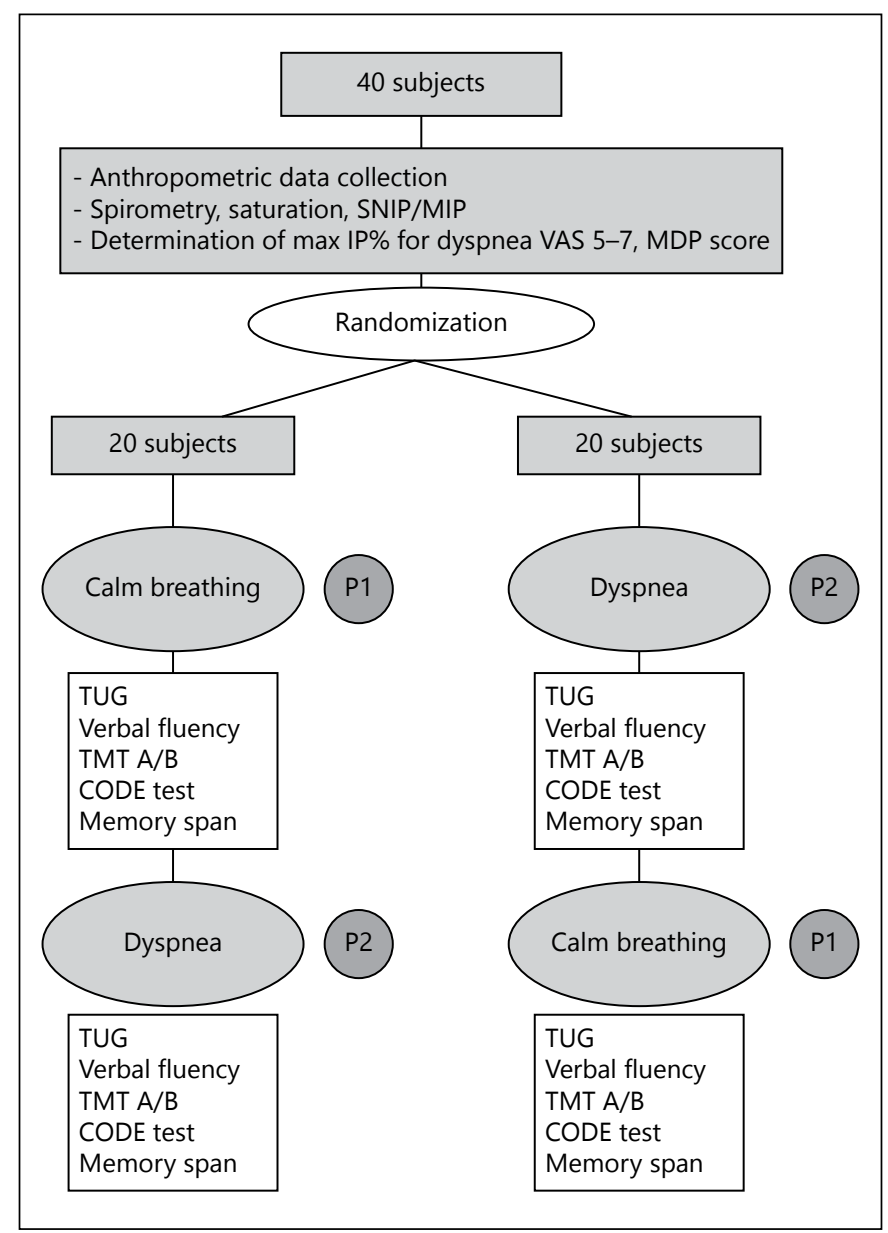

Fig. 2. Flowchart (for Abstract P39).

on cognitive functions and involves the activation of specific cortical areas.

Our study hypothesizes that dyspnea leads to cognitive dysfunction by "cortical competition". We predict that experimentally induced dyspnea will interfere with gait control (primary outcome) and affects cognitive function tests (secondary outcome).

Methods: 40 healthy subjects (Table 1 ) will be randomized to the sequence "calm spontaneous breathing"/"experimental dyspnea" or "experimental dyspnea"/ "calm spontaneous breathing". Dyspnea will be elicited by progressive increase of threshold loading aiming at an intensity of $6 / 10$ on a visual analog scale. During each period of this cross-over trial, the timed up and go test (TUG), as a measure of gait control (Figure 1), and a battery of cognitive function tests will be performed (Figure 2).

Preliminary Results: To date, 10 subjects have been enrolled. Significant differences have been observed for the TUG test and the indirect span memory test (Table 2).

Expected Results and Conclusion: The purpose of this study is to unravel a causal link between dyspnea and cognitive impairment and its impact on gait control. Such demonstration should stimulate caregivers to consider dyspnea as a syndrome responsible for cognitive impairment. Understanding the impact of dyspnea, irrespective of physiological variables, is a necessary step to understand the many facets of chronic respiratory diseases and improve our patients' daily lives.

\section{P40 \\ Effects of Trumpet Playing on Lung Function - A Case-Control Study}

\section{Studer, D. Schumann, A. Siebeneichler, M. Tamm, D. Stolz \\ University Hospital Basel, Basel, Switzerland}

Introduction: Lung function in wind instrument players is a much-discussed topic with indefinite conclusions. It has been a consideration to use the wind instrument as a treatment modality in respiratory illnesses.

Methods: Participants were recruited from wind orchestra or brass bands throughout Switzerland. Participants were excluded if they played a wind instrument other than a trumpet/cornet/flugelhorn. Controls were members of an orchestra, life-long no windinstrument players. Both players and controls were excluded if they suffered from a chronic lung disease other than COPD or 
asthma or had a current respiratory infection. The primary outcome of the study was the difference in $\mathrm{FEV}_{1}$ \%predicted between players and controls. With a significance of 0.005 , a power of $90 \%$ and an unequal sample size ratio 2:1 (players:controls), we calculated a minimum sample size of 92 players and 46 controls.

Results: 99 trumpet/cornet/flughorn players and 48 controls were included in the study. The players played on average $5.2 \pm 4.1$ hours per week and had played their instrument for an average of $27 \pm 14.4$ years. Players were less commonly current smokers $(13 \%$ vs. $21 \%$ ) and male (67\% vs $85 \%$ ) compared to the controls. Players were significantly older than the controls ( 40.5 vs 33.3 years; $\mathrm{p}=$ $0.008)$ and had a significantly higher BMI $\left(25.3 \mathrm{vs} 24.0 \mathrm{~kg} / \mathrm{m}^{2} ; \mathrm{p}=\right.$ $0.037)$. There was no significant difference in $\mathrm{FEV}_{1}$ \%predicted (91.9 vs $94.2 \%, \mathrm{p}=0.316$ ) and FVC \%predicted (89.4 vs 92.6; $\mathrm{p}=$ 0.125 ) between controls and players. More controls had an FeNO value $>25 \mathrm{ppb}$ compared to trumpet players (41\% vs. $19 \%)$, even after adjusting for age and smoking status $(\mathrm{OR}=3.402, \mathrm{p}=0.038)$. There was a significant inverted association between number of years of playing and both a) FVC \%predicted and b) FVC (L) after adjusting for a) smoking status and b) age, gender, height and smoking status.

Conclusion: In our cohort, playing the trumpet/cornet/flugelhorn does not adversely affect $\mathrm{FEV}_{1}$.

\section{P41 \\ Efficacy of a Multiplex Bacterial PCR in Identifying Pathogens in Pleural Empyema}

\section{Franchetti, D. Schumann, M. Tamm, K. Jahn, D. Stolz}

University Hospital Basel, Basel, Switzerland

Introduction: The identification of the pathogens involved in pleural empyema has mainly relied on conventional bacterial culture or single species polymerase chain reaction (PCR), both with relatively low sensitivity. We investigated the efficacy of a commercial multiplex pneumonia PCR assay to identify the pathogens involved in pleural empyema.

Methods: Prospective, monocentric, observational study. Pleural fluid samples were collected from 194 consecutive patients. All samples underwent conventional microbiology at the time of hospitalisation. Commercial multiplex pneumonia bacterial PCR (Curetis Unyvero p55) was performed retrospectively.

Results: The final clinical diagnosis of empyema was made in $31 \%$ of all cases. There was a suspicion of empyema based on the ultrasound findings in 35/189 (18.5\%) cases and of these, 30 cases $(85.7 \%)$ were diagnosed with empyema (16 hosptial-acquired and 14 community-acquired pneumonia). When only taking into account the 20 pathogens included in the multiplex PCR Assay, more pathogens were identified using the multiplex PCR assay than with conventional methods $(23.3 \%(7 / 30)$ vs. $6.7 \%(2 / 30), p=0.008)$. In those cases without suspicion of empyema, 15/154 (9.7\%) had a final diagnosis of empyema. Using the same criteria, conventional methods identified pathogens in $0 / 15(0 \%)$ cases and the multiplex PCR-based assay identified pathogens in 1/15 (6.7\%) cases. When taking into account all available pathogens, 35 pathogens in 20 samples were identified when using conventional methods com- pared to 11 pathogens in 11 samples when using the multiplex PCR-based assay. The most common microorganisms identified in the cases diagnosed with empyema, were anaerobes (31) followed by gram-positive cocci (10) and gram-negative rods (4).

Conclusion: The multiplex PCR-based assay had a higher sensitivity and specificity than conventional microbiology when only the pathogens on the panel were taken into account. A dedicated pleural empyema multiplex PCR panel including anaerobes would be needed to cover most common pathogens involved in pleural infection.

\section{P42 \\ Improved Assessment of Bronchodilator Response Evaluating Spirometric and Plethysmographic Target Parameters as Combined Primary End-Points in Patients with COPD}

\author{
R. Kraemer ${ }^{1}$, M. Leuenberger ${ }^{2}$, L. Kern ${ }^{2}$, M.H. Brutsche ${ }^{2}$, \\ Asthma-COPD-Overlap Syndrome diversification study \\ ${ }^{1}$ Department of Biomedical Research, University of Berne, Bern- \\ Muri, ${ }^{2}$ Clinic of Pneumology, Cantonal Hospital St. Gallen, St. \\ Gallen, Switzerland
}

Background: Response to bronchodilators is currently evaluated by changes in forced expiratory volume at $1 \mathrm{~s}\left(\mathrm{FEV}_{1}\right)$ and/or forced vital capacity FVC) in patients with COPD. Bronchodilators additionally lower peripheral smooth muscle tone leading to a decrease of pulmonary hyperinflation and air trapping. Changes in small airway dysfunction assessed either by the forced expiratory flow between $25 \%$ and $75 \% \mathrm{FVC}\left(\mathrm{FEF}_{25-75}\right)$ or by the effective, specific airway conductance $\left(\mathrm{sG}_{\mathrm{eff}}\right)$ are rarely appreciated. The aim of the present study was to search for parameters characterizing bronchodilator response including changes in peripheral airway function.

Methods: Response to $200 \mathrm{mcg}$ Salbutamol was evaluated by spirometric $\left(\mathrm{FEV}_{1}, \mathrm{FVC}, \mathrm{FEV}_{1} / \mathrm{FVC}, \mathrm{FEF}_{25-75}\right)$ and plethysmographic $\left(\mathrm{sG}_{\mathrm{eff}}\right)$ parameters in $268 \mathrm{COPD}$ patients, including 55 clinically detected patients with asthma-COPD-overlap (ACO), and compared with 84 asthma patients. Reversibility of airway obstruction was assessed using the ATS/ERS criteria, i.e. an increase of $\mathrm{FEV}_{1}$ and/or $\mathrm{FVC} \geq 12 \%$, and $\geq 200 \mathrm{~mL}$, or an improvement of $\mathrm{FEF}_{25-75}$ and/or sG $\mathrm{G}_{\text {eff }} \geq 20 \%$ from baseline.

Results: Bronchodilator response could be demonstrated by ATS/ERS criteria in COPD patients in $19.4 \%$, compared to asthma in $28.6 \%$ and $\mathrm{ACO}$ in $36.4 \%$. An additional response was observed by $\mathrm{sG}_{\text {eff }}$ in $25.7 \%$, by $\mathrm{FEF}_{25-75}$ in $17.2 \%$ resp. Similar additional response were found for asthma and ACO patients. Hence, ATS/ERS criteria failed to demonstrate bronchodilator response compared to $\mathrm{sG}_{\text {eff }}$ in $27.4 \%$ to $\mathrm{FEF}_{25-75}$ in $18.5 \%$ resp.

Conclusions: A significant additional proportion of COPD patients reached reversibility of airway obstruction if improvement of airway dysfunction $\left(\mathrm{sG}_{\mathrm{eff}}, \mathrm{FEF}_{25-75}\right)$ is taken into account. With the aid of mid-expiratory flow characteristics and specific airway conductance a subset of patients could be identified with a small airway reversibility phenotype - potentially indicating ACO. 


\section{P43}

\section{How to Set Up High Frequency Non-Invasive Ventilation (HF-NIV): A Bench Test}

A. Lovis ${ }^{1}$, P. Vremaroiu' ${ }^{1}$, O. Long ${ }^{2}$, J. Simon ${ }^{2}$, L. Noirez ${ }^{1}$, L. Piquilloud ${ }^{3}$, L. Nicod' ${ }^{1}$, A. Ogna ${ }^{1,4}$

${ }^{1}$ Médecine Interne, ${ }^{2}$ Physiothérapie, ${ }^{3}$ Soin Intensif, CHUV-UNIL, Lausanne, ${ }^{4}$ Médecine Interne et Pneumologie, Ospedale di Locarno, Locarno, Switzerland

Introduction: High-frequency noninvasive ventilation (HF$\mathrm{NIV}$ ) is a new ventilation modality allowing to obtain prolonged apneas in non-sedated subjects. In preliminary studies, we showed good tolerance and promising clinical applications for thoracic MRI and radiation therapy. In this bench study, we aimed to in- vestigate the impact of ventilation parameters, circuits and interfaces to the delivered minute ventilation, in order to optimize ventilation efficacy.

Methods: The minute ventilation delivered by a Monsoon III ventilator (Acutronic Medical Systems) was measured in a bench test with a Michigan Lung Simulator, using 9 respiratory frequencies (100 to $500 \mathrm{bpm}), 5$ working pressures ( 0.8 to 1.6 bar), 4 interfaces (Phasitron, Percussionnaire) and 4 different circuits.

Results: Minute ventilation decreased almost linearly by increasing ventilator frequency (Fig. 1) and increased by increasing working pressure (Fig. 2), with a flattening curve in the higher pressure range. The choice of the Phasitron interface had little impact on the ventilation efficacy, however, the single-use Homecare Universal (HC) Phasitron (A50605-D) showed slightly higher delivered volumes for the same working pressure (Fig.1). Of note, the removal of the spring from the Phasitron (to make it MRI-com-
Fig. 1. Minute volume of the 3 phasitrons, dependent on the ventilator frequencies, $\mathrm{SU}=\mathrm{Sin}$ gle Use (A50605-D), with and without spring, Yellow/Red (A50007-12) and Green/Green phasitron working pressure 1 bar (for Abstract P43).

Fig. 2. Minute volume of the 3 phasitrons, dependent on the working pressure, $\mathrm{SU}=$ Single Use, with and without spring, ventilator frequency $250 / \mathrm{min}$. (for Abstract P43).
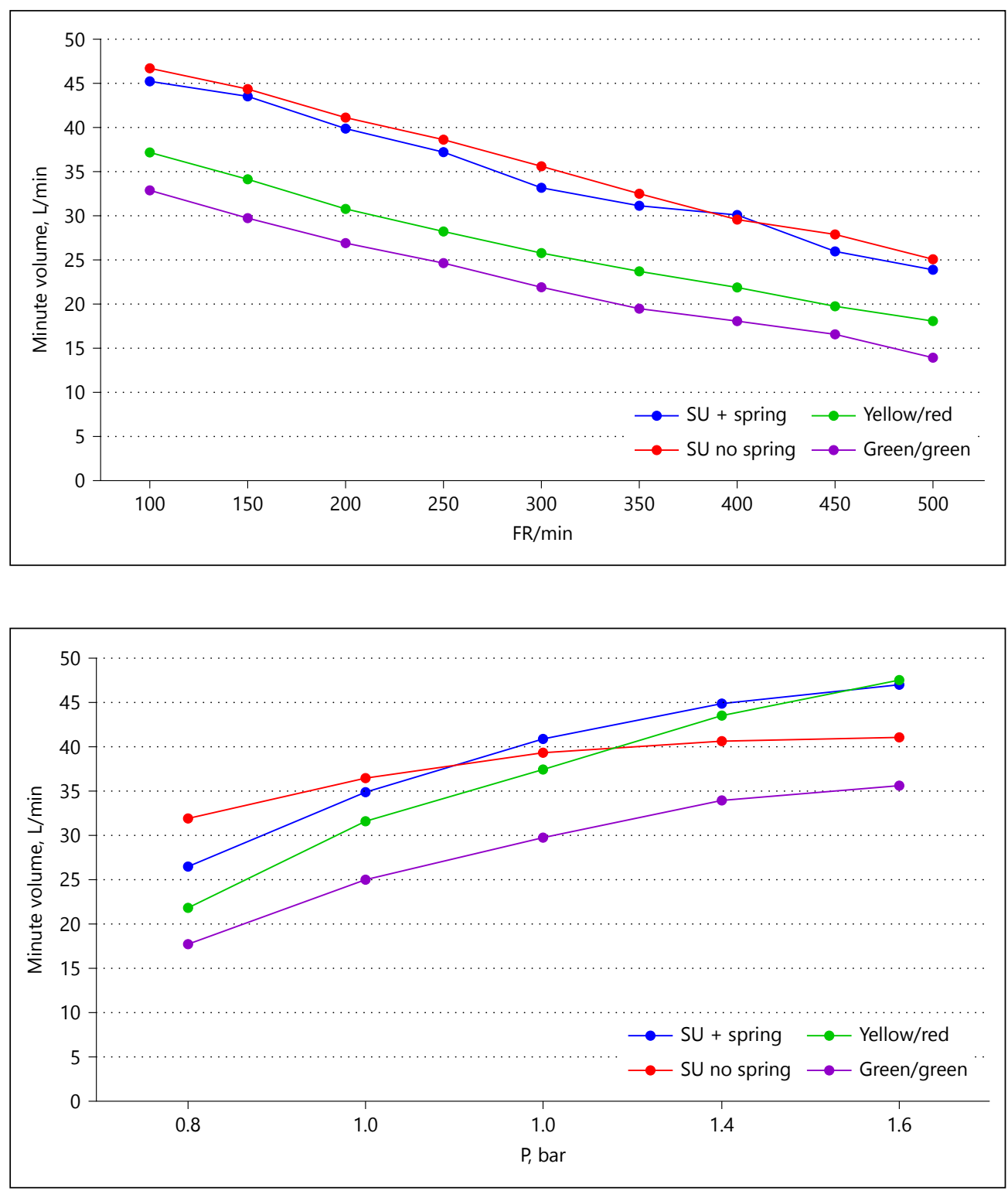
patible) did not modify its performance (Fig.1). A more rigid circuit was associated with a more efficient ventilation, whilst a longer circuit impacted negatively on the delivered volume (Fig. 3 and 4 not upload, only 2 allowed).

Conclusion: Ventilation parameters, circuits and interfaces all seem to have an impact on the ventilation efficacy. The combination of the single-use HC Phasitron, a short and rigid circuit and a ventilation frequency of 250-300 bpm seems to be the most efficient one to obtain tidal volumes just above the anatomical dead space, in order to maintain effective ventilation with the minimal thoracic movements.

\section{P44}

\section{Automated Particle Analysis for Inorganic Lung Dust Characterisation}

\author{
B.K. Kuhn ${ }^{1}$, J. Rausch ${ }^{2,3}$, D. Jaramillo Vogel ${ }^{2,3}$, B. Vrugt ${ }^{1,4}$ \\ ${ }^{1}$ SILAG - Swiss Laboratory for Particle Analysis in Tissues, \\ Zürich, ${ }^{2}$ Particle Vision $\mathrm{GmbH},{ }^{3}$ Département des Géosciences, \\ Université de Fribourg, Fribourg, ${ }^{4}$ Institut für Pathologie USZ, \\ Zürich, Switzerland
}

The cause or agent inducing occupational lung diseases is often difficult to identify. In such cases dust analysis of lung tissue may help. Up to now, inorganic dust particles were analysed manually by transmission electron microscope (TEM). In the last few years automated particle analysis using scanning electron microscope (SEM) was developed. The inorganic dust obtained from lung tissue from two patients was independently analysed by TEM and SEM respectively.

Inorganic dust particles were isolated from freeze-dried lung tissue by low temperature ashing and filtered through polycarbonate filters (Nuclepore) with $0.2 \mu \mathrm{m}$ pore size, which were then carbon coated for analysis.

For manual particle analysis a TEM (Tecnai Spirit, FEI) equipped with an Energy-dispersive X-ray spectrometer (EDX; Oxford Instruments) at the University of Zurich was used. The automated particle analysis was performed with a SEM (Zeiss GeminiSEM 300) at the Particle Vision laboratory equipped with an Oxford X-MAX $80 \mathrm{~mm}^{2}$ EDX detector and the Oxford AZtec Feature software.

Table 1. Overview on particle occurrence (for Abstract P44)

\begin{tabular}{lrrrr}
\hline Particle composition & TEM 1 & SEM 1 & TEM 2 & SEM 2 \\
\hline Quartz & $23 \%$ & $13 \%$ & $21 \%$ & $8 \%$ \\
Other silicates & $43 \%$ & $58 \%$ & $14 \%$ & $17 \%$ \\
Aluminium oxide & $6 \%$ & $2 \%$ & $0 \%$ & $0 \%$ \\
Titanium oxide & $17 \%$ & $14 \%$ & $21 \%$ & $23 \%$ \\
Iron oxide ( $\pm \mathrm{Ti}, \mathrm{Cr}, \mathrm{Ni}, \mathrm{Si})$ & $8 \%$ & $7 \%$ & $5 \%$ & $8 \%$ \\
P-compounds $( \pm \mathrm{Fe}, \mathrm{Na}, \mathrm{Si})$ & $2 \%$ & $5 \%$ & $21 \%$ & $26 \%$ \\
P-compounds with Ti & $0 \%$ & $0 \%$ & $17 \%$ & $17 \%$ \\
Copper sulfide & $2 \%$ & $1 \%$ & $<1 \%$ & $<1 \%$ \\
Number of analysed particles & 121 & 2407 & 211 & 3462 \\
\hline
\end{tabular}

Case 1: A 61 year old male with interstitial lung fibrosis. Two thirds of the 121 particles analysed by TEM were classified as silicate minerals, $30 \%$ as oxides and $2 \%$ as phosphor compounds. With automated SEM-EDX analysis, 2407 particles were analysed delivering a comparable result (Table 1).

Case 2: A 23 year old male with pneumonia. Histologic examination showed abundant birefringent material in lung tissue. Of 211 analysed particles in TEM 35\% were recognized as silicate minerals, $21 \%$ as titanium oxide and $38 \%$ as phosphor compounds. The predominance of phosphor compounds (43\%) was confirmed by automated SEM analysis.

The evaluation of the automated particle analysis by SEM-EDX showed a good reproducibility of the TEM analysis with the advantage of considerably higher numbers of analysed particles guarantying a better statistical representativeness of the results without the need of an operator during the analysis.

\section{Poster Discussion Posters}

\section{P45 \\ Spirometry in Central Asian Lowlanders and Highlanders, a Population Based Study}

\author{
S. Ulrich' ${ }^{1}$, M. Furian' ${ }^{1}$, B. Estebesova ${ }^{2}$, N. Toktogulova ${ }^{2}$, \\ G. Beishekeeva ${ }^{3}$, S. Ulrich ${ }^{1}$, P.G.J. Burney', T.M. Sooronbaev', \\ K.E. Bloch ${ }^{1}$
}

'Department of Respiratory Medicine, University Hospital Zurich, Zurich, Switzerland, ${ }^{2}$ Department of Respiratory Medicine, National Center for Cardiology and Internal Medicine, ${ }^{3}$ Kyrgyz-Russian Slavic Univeristy, Bishkek, Kyrgyzstan, ${ }^{4}$ National Heart and Lung Institute \& MRC-PHE Centre for Environment and Health, Imperimental College London, London, United Kingdom

Introduction: The purpose of the study was to establish spirometric reference values for a Central Asian population of highlanders and lowlanders.

Methods: Spirometries from a population-based cross-sectional study performed in 2013 in Kyrgyzstan (Burden Of Lung Disease survey, BOLD: Buist et al. COPD 2005) were analyzed. Using multiple linear regression, Global Lung Function Initiative (GLI) equations were fitted separately for men and women, and altitude of residence (lowlands 700-800 m, highlands 1900$2800 \mathrm{~m}$ ) to data from healthy, never-smoking Kyrgyz adults. The general GLI equation was applied: predicted value $=\exp (\mathrm{a} 0+$ $\mathrm{a} 1 * \ln ($ height $)+\mathrm{a} 2 * \ln ($ age $)+\mathrm{b} 1^{*}($ age $/ 100)+\mathrm{b} 2 *($ age $/ 100) \wedge 2+$ $\left.\mathrm{b} 3 *(\text { age } / 100)^{\wedge} 3+\mathrm{b} 4^{*}(\text { age } / 100)^{\wedge} 4+\mathrm{b} 5 *(\text { age } / 100)^{\wedge} 5\right)$.

Results: Of 2784 Kyrgyz in the BOLD survey, 448 highlanders (379 females) and 505 lowlanders (368 females) met inclusion criteria. Prediction equations for FVC in the Kyrgyz sample fit best with the GLI "North-East Asian" whereas equations for FEV1 fit best with "Other/Mixed" category. Predicted FEV1/FVC was low- 
er than that of all GLI categories. Compared to GLI "Caucasians", Kyrgyz females and males (lowlanders and highlanders combined) have lower age- and height-adjusted FVC: mean difference -0.104 1, -0.112 l, FEV1: -0.174 l, $-0.201 \mathrm{l}$ and FEV1/FVC: $-0.032,-0.029$ ( $\mathrm{P}<0.001$, all comparisons), respectively. Among Kyrgyz, age- and sex-adjusted mean FEV1, FVC and PEF are higher in highlanders $(+0.132 \mathrm{l},+0.138 \mathrm{l}$ and $+0.460 \mathrm{l} / \mathrm{s})$ than in lowlanders $(\mathrm{P}<0.001$, all comparisons), but FEV1/FVC is similar.

Conclusion: We established prediction equations for an adult Central Asian population indicating that FVC is similar to the GLI category "North-East Asian" and FEV1/FVC is lower than in all other GLI categories. Kyrgyz highlanders have significantly higher predicted FVC, FEV1 and PEF than lowlanders.

\section{P46 \\ Effect of Endoscopic Lung Volume Reductions in Patients with Emphysema}

\section{F. Baty, T. Schneider, K. Kozu, L. Kern, M. Brutsche}

Pulmonary and Sleep Medicine, Cantonal Hospital St. Gallen, St. Gallen, Switzerland

Introduction: Endoscopic lung volume reduction (ELVR) is a commonly accepted treatment for patients with severe emphysema. We performed a retrospective study in order to assess the effect of ELVR on patients with emphysema.

Methods: 65 patients with emphysema were referred from January 2010 to December 2017 to the Department of Pulmonary Medicine of the Cantonal Hospital St. Gallen for an ELVR. Lung function including forced expiratory volume in 1 second (FEV1), maximal vital capacity (VCmax), forced vital capacity (FVC), residual volume (RV), total lung capacity (TLC) and diffusing capacity (DLCO) together with parameters of exercise capacity (6-min walking distance) were collected and analyzed using linear mixed models.

Results: Patients had a median age of 67 (IQR: 62.5-72) and a pre-operative FEV1 of $28 \%$ (sd: 8.80 ) and residual volume of $213 \%$ (sd: 41.57). Intrabronchial valves were used in 43 patients, whereas coil implantation was used in 21 patients. One patient received consecutively valves and coils. The effect of ELVR 3 months within insertion included a significant decrease of $0.36 \mathrm{~L}$ of TLC ( $\mathrm{p}=$ $0.001)$ and $0.40 \mathrm{~L}$ of RV ( $\mathrm{p}=0.004)$, as well as a significant increase of $5.3 \%$ of VCmax $(\mathrm{p}=0.029)$ and an improved exercise capacity (59 $\mathrm{m}$ improvement of the 6-min walking distance, $\mathrm{p}=0.043$ ). A significant improvement of $0.37 \mathrm{~L}$ of FVC was also observed 6 months after intervention.

Conclusion: There was a significant short-term improvement in lung function following ELVR in patients with emphysema. The effect was observed within 6 months after therapy.

\section{P47 \\ Cardiovascular and Metabolic Correlates of Abnormal Spirometry (Pneumolaus Study)}

\section{A. Lenoir ${ }^{1}$, P.M. Marques-Vidal ${ }^{2}$, P. Vollenweider ${ }^{2}$, J.-W. Fitting ${ }^{1}$, L.P. Nicod ${ }^{1}$}

${ }^{1}$ Service de Pneumologie, ${ }^{2}$ Service de Medecine Interne, Centre Hospitalier Universitaire Vaudois, Lausanne, Switzerland

Patients with impaired lung function frequently present with comorbidities further affecting their disease burden. We evaluated the association of cardiovascular risk factors with abnormal lung function in a sample of the general population of Lausanne, Switzerland.

Between 2014 and 2017, we examined 3351 participants of the CoLaus study, a prospective study investigating the occurrence of cardiovascular diseases and risk factors in the general population of Lausanne. Spirometry was performed according to ATS/ERS 2005 guidelines and interpreted using GLI 2012 references. If FEV1/FVC or FVC was below the Lower Limit of Normal (LLN), spirometry was repeated after bronchodilation (BD). Chronic airway obstruction was defined as FEV1/FVC $<$ LLN after BD; reversible airway obstruction as FEV1/FVC or FVC $<$ LLN before BD and $>\mathrm{LLN}$ after $\mathrm{BD}$; possible lung restriction as $\mathrm{FVC}<\mathrm{LLN}$ after BD.

Among 3351 participants, 91.9\% had normal spirometry, 2.4\% reversible and $3.5 \%$ chronic obstruction; $1.8 \%$ had possible lung restriction without obstruction, $0.4 \%$ with chronic, $<0.1 \%$ with reversible obstruction. In participants with abnormal spirometry, prevalence of hypertension $(52.9 \%$ vs. $43.3 \%, \mathrm{p}=0.002)$ and diabetes $(16.2 \%$ vs. $8.7 \%, \mathrm{p}<0.0001)$ was significantly higher. Particularly, subjects with restrictive pattern often suffered from hypertension (68.0\%) and diabetes (33.3\%). Similarly, glucose (5.9vs. $5.4 \mathrm{mmol} / \mathrm{l}, \mathrm{p}=0.0001)$ and insulin levels ( $12.7 \mathrm{vs} .9 .7 \mu \mathrm{IU} / \mathrm{ml}, \mathrm{p}=$ $0.0304)$ were significantly higher in the restrictive group. These findings were not confirmed for obstruction and independent of smoking status except for insulin levels. Although mean BMI was higher in the restrictive group $\left(28.7 \pm 7.0\right.$ vs. $26.3 \pm 4.6 \mathrm{~kg} / \mathrm{m}^{2}, \mathrm{p}<$ 0.0001 ), prevalence of hypertension but not diabetes remained significantly increased when analysing normal weight subjects only.

We conclude that abnormal lung function, particularly restrictive spirometric pattern, is associated with increased prevalence of hypertension and diabetes. Identifying subjects with restrictive ventilatory pattern seems of high clinical and public health importance. 


\section{P48}

\section{Strong Increase in the Prevalence of COPD in Switzerland between 2003 and 2012}

\section{A. Strassmann ${ }^{1}$, E. West ${ }^{1}$, C. Wang ${ }^{2}$, A. Turk ${ }^{3}$, K. De Hogh ${ }^{4}$, M. Röösli ${ }^{4}$, M. Bopp ${ }^{1}$, H. Dressel ${ }^{1}$, M. Puhan ${ }^{1}$, Swiss National Cohort Study Group}

${ }^{1}$ Epidemiology, Biostatistics und Prevention Institute, University of Zurich, ${ }^{2}$ Department of Mathematics, University of Zurich, Zurich, ${ }^{3}$ See-Spital Horgen, Horgen, ${ }^{4}$ Swiss Tropical and Public Health Institute, Basel, Switzerland

Introduction: Most studies to determine the prevalence of COPD are conducted only once. Our aim was to determine the prevalence of COPD yearly in general population samples between 2003 and 2012.

Methods: We based our analysis on data from 38,390 participants aged $\geq 35$ years who received a lung function measurement as part of the LuftiBus health promotion campaign of Lunge $\mathrm{Zu}$ rich. The LuftiBus represents the adult Swiss population well and its data have been used to derive population-based lung function reference equations. Spirometry was performed using computerized pneumotachographs by trained staff, according to American Thoracic Society guidelines but without bronchodilation (not feasible in LuftiBus). We used both lower limit of normal (LLN) and FEV1/FVC of $<0.7$ to determine the presence of COPD. We used the linkage to the Swiss National Cohort to adjust for potential differences between yearly population samples.
Results: While the population samples were very similar from year to year, the prevalence of COPD increased between 2003-2012 from $9.3 \%$ to $17.9 \%$ (based on LLN, higher estimates if based on FEV1/FVC). FVC was stable (median across years 3.9 liter) over time. The increase in prevalence affected both ever and never smokers and was observed for mild but not moderate to severe airflow obstruction (Figure). The increase in COPD prevalence remained after adjusting for age, sex, body mass index, smoking status, distance of residence to main road, educational status, air pollution as well as temperature at the time of measurement.

Discussion: Although pre-bronchodilator measurements likely overestimate the prevalence of COPD in absolute terms, we found a strong increase in prevalence. Since the trend existed in both ever and never smokers and is only observable for mild COPD, the explanation may be found in prevalent exposures 30 or 40 years ago like air pollution, which peaked in the 1970s.
Fig. 1. Prevalence of COPD and of severity of airflow obstruction between 2003 and 2012 (for Abstract P48).

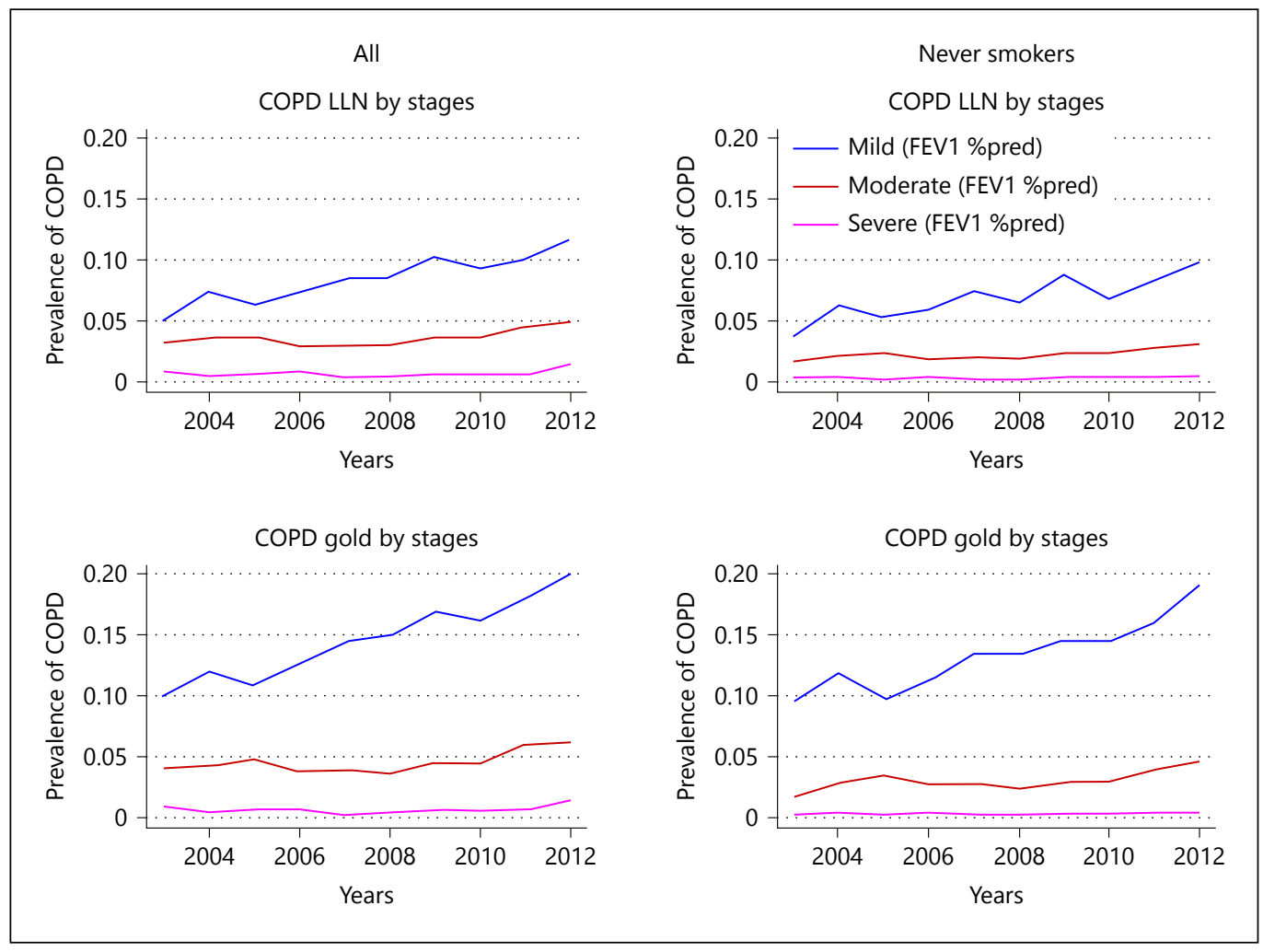




\section{P49 \\ Stability of the Eosinophilic Phenotype in Stable and Exacerbated Chronic Obstructive Pulmonary Disease}

\section{Schumann, M. Tamm, K. Kostikas, D. Stolz}

University Hospital Basel, Basel, Switzerland

Introduction: There is controversy around the use of blood eosinophil levels as a biomarker in determining the responsiveness of patients to inhaled corticosteroids (ICS). Studies have shown that a blood eosinophil level $\geq 2 \%$ may be a predictor for response to ICS treatment and that patients with blood eosinophils $>300$ cells/ $\mu \mathrm{l}$ are at a higher risk of severe exacerbations. We investigated the stability of blood eosinophils in COPD patients in stable state and during exacerbation.

Method: This is a nested study of the PROMISE-COPD study where patients with stable COPD stage GOLD II to IV were enrolled. Only data from patients with more than one visit were used for the current analysis. Concordance or stability was defined as blood eosinophil levels persistently lower than or persistently higher than the absolute cut-off points 150 cells/ $\mu$ l, 300 cells/ $\mu$ l or the percentage cut-off points $2 \%, 3 \%$, and $4 \%$. Discordance was obtained when the blood eosinophil levels varied between any two visits dichotomized by stable state or exacerbation status.

Results: We analysed data from 230 patients with 1557 visits during the stable phase of COPD and 522 visits with exacerbation. The median follow up period was 4.6 years. The median blood eosinophil level during stable state was 183 cells/ $\mu$ l, during mild/ moderate exacerbation 169 cells/ $\mu$ l and during severe exacerbation 131 cells/ $\mu$ l. There was an overall high degree of discordance of up to $60 \%$ during mild/moderate exacerbation, $63 \%$ during severe exacerbation and up to $76 \%$ during stable state. $84.8 \%$ of the patients received ICS at the beginning of the study. The stability of the blood eosinophil was not associated with ICS treatment.

Conclusion: Our data suggest that blood eosinophil levels vary significantly throughout the course of COPD and therefore a single measurement may not be a reliable predictor of ICS response.

\section{P50 \\ Single-Port Thymectomy Using a Subxiphoid Approach}

\section{B. Bédat, S.M. Sadowski, F. Triponez, W. Karenovics \\ Thoracic and Endocrine Surgery Unit, Geneva University Hospitals, Geneva, Switzerland}

Background: Thymectomy is currently performed by a lateral thoracic approach. However, this approach can cause difficulties to identify the contralateral phrenic nerve. A subxiphoid approach could permit to visualize the bilateral phrenic nerves and to decrease the postoperative pain. The aim of this study is to assess the feasibility of the single-port thymectomy using a subxiphoid approach and to evaluate the early surgical outcomes.

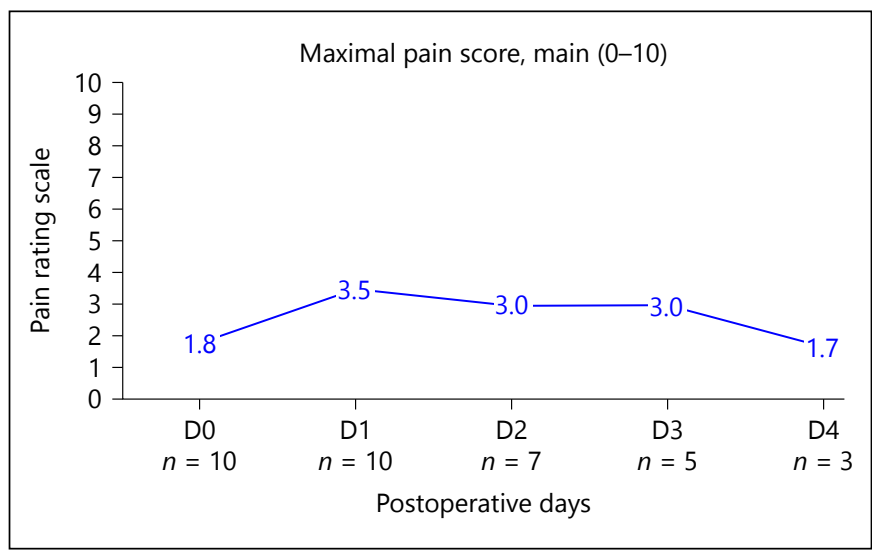

Fig. 1. Postoperative pain (for Abstract P50).

Methods: We retrospectively reviewed all consecutive patients who underwent single-port subxiphoid thymectomy from December 2015 to December 2017 at University Hospitals of Geneva. Patient characteristics, indications, pathology findings and early postoperative outcomes were analyzed.

Results: Ten patients ( 4 male, 6 women) with a mean age of $43 \pm 15$ years old underwent a single-port thymectomy using a subxiphoid approach. Indications for thymectomy was : myasthenia gravis $(\mathrm{n}=8)$, thymoma-associated myasthenia gravis $(\mathrm{n}=1)$ and parathyroid adenoma $(n=1)$. The mean operation time was $126 \pm 40$ minutes. There was no complication during the operation and bilateral phrenic nerves were identified in all patients. No patient died during the postoperative period. One complication was identified and consisted to a postoperative myasthenic crisis. Intraoperative drain removal was performed in three patients. The median duration of drainage was 1 day (range $0-1$ day) and the median length of hospitalization was 2 days (range 1-13 days). The mean of maximal postoperative pain during the early postoperative period was shown in Figure 1. The intraoperative drain removal was not correlated to a decreased postoperative pain.

Conclusion: Single-port thymectomy using a subxiphoid approach is safe, causes postoperative low pain, and permits the identification of the bilateral phrenic nerves.

\section{P51 \\ Analysis of Hospitalizations for Tuberculosis in Switzerland from 2002 to 2015}

\author{
$\underline{\text { S. Tonko }}^{1}$, F. Baty ${ }^{1}$, M. Brutsche ${ }^{1}$, O.D. Schoch ${ }^{1,2}$ \\ ${ }^{1}$ Klinik für Pneumologie und Schlafmedizin, Kantonsspital \\ St.Gallen, St.Gallen, ${ }^{2}$ Lungenliga Schweiz, Kompentenzzentrum \\ Tuberkulose, Bern, Switzerland
}

Introduction: Historically, patients with tuberculosis have been hospitalized for several months. Today, Swiss guidelines recommend outpatient management or discharge of patients from 


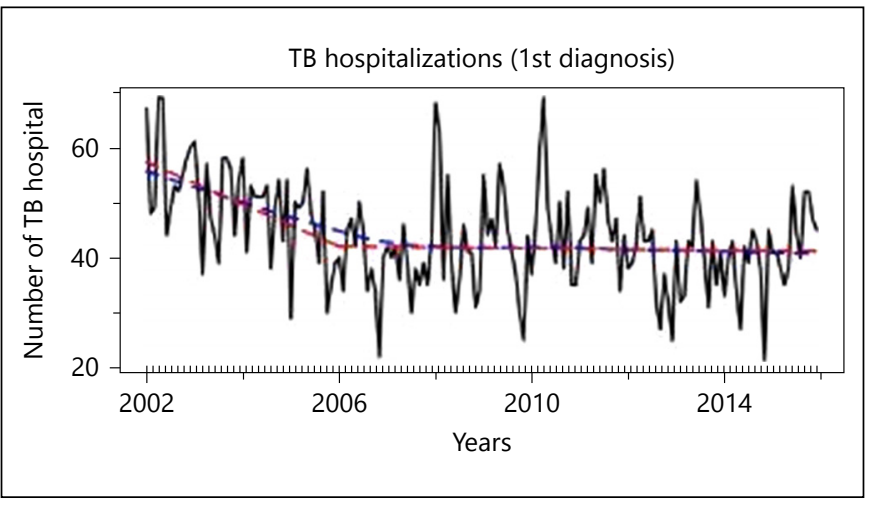

Fig. 1. Trend and Monthly Fluctuations of Tuberculosis Hospitalizations in Switzerland from 2002-2015 (for Abstract P51).

hospital as quickly as possible, whenever permissible. The aim of this retrospective analysis was to describe the rate of hospitalizations and length of stay (LOS) of patients with tuberculosis for the years 2002-2015 in Switzerland.

Methods: Data were extracted from a nation-wide database provided by the Swiss Federal Office for Statistics enclosing 19,832,602 hospital entries between 2002 and 2015. Hospitalizations were retrieved by ICD-10 codes (A15-A19). A total of 8047 primary and $14041 \mathrm{co}$-diagnoses were identified. The number of hospitalizations were compared to the annual tuberculosis cases reported to the Swiss Federal Office of Public Health. Subgroupanalyses were performed including HIV and diabetes. Differences between miliary, non-miliary and extrapulmonary tuberculosis were investigated.

Results: Reported cases and hospitalisations were similar with a significant decline from 673 hospitalizations/year in 2002 to 471 in 2006 ( $p<0.001)$ was followed by a stabilization $(p=0.309$, Figure 1). The median LOS was 14 days (IQR: 6-22) and did not change over time $(\mathrm{p}=0.073)$. In-hospital mortality was $2.3 \%$ and did not change over time ( $\mathrm{p}=0.430)$. The age distribution was bimodal with a first peak between $20-30$ and another peak at 70-90 years-old. The older group ( $>50$ years-old) stayed 1 day longer ( 14 [IQR: 5-20] vs. 15 days [IQR: 8-26]), however in-hospital mortality increased from $0.5 \%$ to $5.7 \%$ in the older. Patients with miliary tuberculosis (vs. pulmonary) had a longer LOS (median 16 days vs. 20 days, $\mathrm{p}<0.001$ ) and a higher in-hospital mortality (median $2.3 \%$ vs. $6.9 \%, \mathrm{p}<0.001)$.

Conclusion: Few cases have been treated in outpatient settings. Tuberculosis hospitalizations decreased from 2002-2006, followed by a stabilization between 2006 and 2015. Mortality and comorbidities did not change. LOS and mortality in miliary tuberculosis was significantly higher.

\section{P52 \\ Effect of Endoscopic Lung Volume Reductions in Patients with Emphysema}

\section{F. Baty, T. Schneider, K. Kozu, L. Kern, M. Brutsche}

Pulmonary and Sleep Medicine, Cantonal Hospital St. Gallen, St. Gallen, Switzerland

Introduction: Endoscopic lung volume reduction (ELVR) is a commonly accepted treatment for patients with severe emphysema. We performed a retrospective study in order to assess the effect of ELVR on patients with emphysema.

Methods: 65 patients with emphysema were referred from January 2010 to December 2017 to the Department of Pulmonary Medicine of the Cantonal Hospital St. Gallen for an ELVR. Lung function including forced expiratory volume in 1 second (FEV1), maximal vital capacity (VCmax), forced vital capacity (FVC), residual volume (RV), total lung capacity (TLC) and diffusing capacity (DLCO) together with parameters of exercise capacity (6-min walking distance) were collected and analyzed using linear mixed models.

Results: Patients had a median age of 67 (IQR: 62.5-72) and a pre-operative FEV1 of $28 \%$ (sd: 8.80 ) and residual volume of $213 \%$ (sd: 41.57). Intrabronchial valves were used in 43 patients, whereas coil implantation was used in 21 patients. One patient received consecutively valves and coils. The effect of ELVR 3 months within insertion included a significant decrease of $0.36 \mathrm{~L}$ of TLC $(\mathrm{p}=$ $0.001)$ and $0.40 \mathrm{~L}$ of RV ( $\mathrm{p}=0.004)$, as well as a significant increase of $5.3 \%$ of VCmax $(\mathrm{p}=0.029)$ and an improved exercise capacity (59 $\mathrm{m}$ improvement of the 6 -min walking distance, $\mathrm{p}=0.043$ ). A significant improvement of $0.37 \mathrm{~L}$ of FVC was also observed 6 months after intervention.

Conclusion: There was a significant short-term improvement in lung function following ELVR in patients with emphysema. The effect was observed within 6 months after therapy.

\section{P53 \\ Lung Function in Birt-Hogg-Dubé Syndrome}

C. Daccord ${ }^{1}$, R. Borie ${ }^{2}$, M. Faouzi ${ }^{3}$, C. Khouatra 4 , A. Gondouin 5 , A. Briault ${ }^{6}$, J.-F. Cordier ${ }^{4}$, V. Cottin ${ }^{4}$, R. Lazor ${ }^{1,4}$

${ }^{1}$ Respiratory Medicine Department, Lausanne University Hospital, Lausanne, Switzerland, ${ }^{2}$ Respiratory Medicine Department, Bichat Claude-Bernard University Hospital, Paris, France, ${ }^{3}$ Institute of Social and Preventive Medicine, Lausanne University Hospital, Lausanne, Switzerland, ${ }^{4}$ Reference Centre for Rares Pulmonary Diseases, Claude Bernard University Hospital, Lyon, ${ }^{5}$ Respiratory Medicine Department, Besançon University Hospital, Besançon, ${ }^{6}$ Respiratory Medicine

Department, Grenoble University Hospital, Grenoble, France

Introduction: Birt-Hogg-Dubé syndrome (BHDS) is a rare inherited autosomal dominant disorder characterized by cutaneous fibrofolliculomas, renal tumors, multiple pulmonary cysts and 
spontaneous pneumothoraces. BHDS is caused by mutations in the tumor suppressor gene FLCN coding for folliculin. Only limited data on lung function in BHDS are available, and whether lung function declines over time as in other cystic lung diseases is unknown.

Aims: To assess lung function at baseline and over time in BHDS.

Methods: We retrospectively studied lung function parameters from 30 patients with BHDS at baseline and during disease course, and looked for correlations with age, gender and smoking history.

Results: Mean \pm SD age at baseline was $42 \pm 14 \mathrm{yr}, 60 \%$ were males, $43 \%$ were smokers and $93 \%$ had pulmonary cysts at HRCT. Baseline FEV1 and FVC $(\mathrm{n}=30)$ were $95 \pm 13$ and $100 \pm 14 \%$ pred respectively, and FEV1/FVC ratio was $80 \pm 6 \%$. TLC $(n=21)$ was $98 \pm 15 \%$ pred, RV $(\mathrm{n}=21)$ was $111 \pm 30 \%$ pred, DLCO $(\mathrm{n}=18)$ was $86 \pm 16 \%$ pred, $\mathrm{KCO}(\mathrm{n}=19)$ was $96 \pm 20 \%$ pred, and $\mathrm{PaO} 2(\mathrm{n}=$ 8 ) was $88 \pm 11 \mathrm{~mm} \mathrm{Hg}$. FEV1, FVC and FEV1/FVC at 1, 2 and 3 years follow-up ( $\mathrm{n}=12,9$ and 6 respectively) were not significantly different from baseline. FEV1/FVC tended to decrease with age at baseline $(\mathrm{p}=0.05)$ and over time $(\mathrm{p}=0.02)$. In smokers, FEV1 was $9 \%$ lower at baseline $(p=0.08)$ and over time $(p=0.013)$ as compared to non-smokers. FVC significantly increased with age at baseline $(\mathrm{p}=0.007)$ and over time $(\mathrm{p}=0.002)$. Other parameters at baseline and over time were not correlated with age, gender and smoking history.

Conclusions: This preliminary analysis on 30 patients suggest that cystic lung disease in BHDS does not affect respiratory func- tion at baseline, and that no deterioration occurs over a follow-up period of 3 years. The data of approximately 50 additional patients are currently being analysed.

\section{P54 \\ Respiratory Viral Infection in Immunocompromised Patients}

K. Jahn ${ }^{1}$, D. Schumann ${ }^{1}$, M. Tamm ${ }^{1}$, H. Hirsch ${ }^{2}$, J. Halter ${ }^{3}$, L. Junker ${ }^{1}$, W. Strobel', S. Savic ${ }^{4}$, D. Stolz ${ }^{1}$

${ }^{1}$ Clinic of Pneumology and Pulmonary Cell Research,

${ }^{2}$ Transplantation and Clinical Virology, ${ }^{3}$ Department of Haematology, ${ }^{4}$ Department of Pathology, University Hospital Basel, Basel, Switzerland

Introduction: Knowledge on viral infections in immunocompromised patients is sparse. We investigated the incidence of respiratory viruses in a well-characterized population of immunocompromised patients.

Methods: All immunocompromised patients with suspicion of infection and/or respiratory symptoms and/or radiologically confirmed lung infiltrates undergoing bronchoscopy were included in the study. In addition to the routine assessment of bronchoalveolar

Table 1. Incidence of respiratory viruses (for Abstract P54)

\begin{tabular}{lccc}
\hline Respiratory viruses & $\begin{array}{l}\text { Haematological Group } \\
\text { [virus pos. } \mathrm{n}=374] \\
\mathrm{n}(\%)\end{array}$ & $\begin{array}{l}\text { Solid organ } \\
\text { Transplantation Group } \\
\text { [virus pos. } \mathrm{n}=261 \text { ] } \mathrm{n}(\%)\end{array}$ & $\begin{array}{l}\text { 'Other' Group } \\
\text { [virus pos. } \mathrm{n}=301] \\
\mathrm{n}(\%)\end{array}$ \\
\hline Adenovirus & $18(4.8)$ & $4(1.5)$ & $6(2.0)$ \\
Bocavirus & $3(0.80)$ & $4(1.5)$ & $3(1.0)$ \\
Coronavirus & $32(8.6)$ & $23(8.8)$ & $28(9.3)$ \\
Human metapneumovirus & $34(9.1)$ & $17(6.5)$ & $11(3.7)$ \\
H1N1 & $4(1.1)$ & $16(6.1)$ & $0(0)$ \\
Influenza & $26(7.0)$ & $16(6.1)$ & $15(5.0)$ \\
Parainfluenza & $54(14.4)$ & $107(6.9)$ & $24(8.0)$ \\
Rhinovirus-Enterovirus & $124(33.2)$ & $20(7.7)$ & $100(33.2)$ \\
Respiratory syncytial virus & $34(9.1)$ & $23(7.6)$ \\
\hline
\end{tabular}

Table 2. Incidence of other viruses (for Abstract P54)

\begin{tabular}{llll}
\hline Other viruses & $\begin{array}{l}\text { Haematological } \\
\text { Group [Virus pos. } \\
\mathrm{n}=374] \mathrm{n}(\%)\end{array}$ & $\begin{array}{l}\text { Solid organ } \\
\text { Transplantation Group } \\
\text { [Virus pos. } \mathrm{n}=261] \mathrm{n}(\%)\end{array}$ & $\begin{array}{l}\text { 'Other' Group } \\
\text { [Virus pos. } \mathrm{n}=301] \\
\mathrm{n}(\%)\end{array}$ \\
\hline Cytomegalovirus & $84(22.5)$ & $70(26.8)$ & $81(26.9)$ \\
Epstein-Barr virus & $7(1.9)$ & $0(0)$ & $1(0.33)$ \\
Herpes simplex virus & $44(11.8)$ & $22(8.4)$ & $0(17.3)$ \\
Human Herpes virus & $4(1.1)$ & $0(0)$ & $0(0)$ \\
Varicella-zoster virus & $1(0.27)$ & $0(0)$ & \\
\hline
\end{tabular}


lavage fluid, a multiplex PCR for 20 viruses has been performed. Study period was from October 2009 to September 2017.

Results: 1303 patients with 2666 samples were included in the study. $35 \%(n=936)$ of the samples were positive for viral infection, $23 \%(n=610)$ were positive for bacterial infection, $1.6 \%(n=$ 43) were positive for atypical mycobacteria, and $10 \%(n=269)$ were positive for fungal infection. Bacterial coinfection was evident in $26 \%(\mathrm{n}=242 / 936)$ and fungal coinfection in $13 \%(\mathrm{n}=$ $117 / 936)$ of the virus positive samples. The incidence of respiratory viruses in the virus positive samples was as follows: adenovirus $3.0 \%(\mathrm{n}=28)$, bocavirus $1.1 \%(\mathrm{n}=10)$, coronavirus $8.9 \%(\mathrm{n}=$ $83)$, human metapneumovirus $6.6 \%(\mathrm{n}=62), \mathrm{H} 1 \mathrm{~N} 10.53 \%(\mathrm{n}=5)$, influenza $6.1 \%(n=57)$, parainfluenza $10.3 \%(n=96)$, rhinovirusenterovirus $35.4 \%(n=331)$, RSV $8.2 \%(n=77)$. Other viruses included cytomegalovirus $25.1 \%(n=235)$, Epstein-Barr virus $0.85 \%$ $(\mathrm{n}=8)$, herpes simplex virus $12.6 \%(\mathrm{n}=118)$, human herpes virus-6 $0.32 \%(n=3)$, human herpes virus-7 $0.1 \%(n=1)$ and varicella-zoster virus $0.1 \%(n=1)$. In one case $(0.1 \%)$, four viruses were identified, in 15 cases (1.6\%) 3 viruses, in 146 cases (15.6\%) 2 viruses and in 774 cases $(82.7 \%)$ one virus was identified. Patients were divided into groups according to the cause of immunosuppression. In the haematological group $36.1 \%(n=374 / 1035)$, in the solid organ transplantation group $39.4 \%(n=261 / 663)$ and in the 'Other' group $31.1 \%(\mathrm{n}=301 / 968)$ of the samples were positive for viral infection (Table 1, 2).

Conclusion: There is a high prevalence of respiratory virus infections in immunocompromised patients undergoing diagnostic bronchoscopy for respiratory symptoms. This finding could influence patient management.

\section{P55 \\ Decline of Physical Activity is Not Explained by Progression of Exercise Intolerance in COPD}

C. Clarenbach ${ }^{1}$, N. Sievi ${ }^{1}$, T. Brack ${ }^{2}$, M. Brutsche ${ }^{3}$, M. Frey ${ }^{4}$, S. Irani ${ }^{5}$, J. Leuppi $i^{6,7}$, R. Thurnheer ${ }^{8}$, M. Kohler ${ }^{9,10}$

${ }^{1}$ Pulmonology, University Hospital Zurich, Zurich, ${ }^{2}$ Cantonal Hospital of Glarus, Glarus, ${ }^{3}$ Cantonal Hospital St. Gallen, St. Gallen, ${ }^{4}$ Clinic Barmelweid, Barmelweid, ${ }^{5}$ Cantonal Hospital of Aarau, Aarau, ${ }^{6}$ Cantonal Hospital Baselland, Liestal, ${ }^{7}$ University of Basel, Basel, ${ }^{8}$ Cantonal Hospital of Münsterlingen, Münsterlingen, ${ }^{9}$ University Hospital Zurich, ${ }^{10}$ Zurich Centre for Integrative Human Physiology, University of Zurich, Zurich, Switzerland

Introduction: Daily physical activity (PA) and exercise capacity is reduced in patients with COPD. Whether the natural longitudinal course of both goes in parallel or one precedes the other is currently unclear.

Methods: In a heterogeneous cohort of COPD patients we annually investigated two exercise capacity tests (1-minute sit to stand test (STS) and 6 minutes walking test (6MWT)) and daily physical activity assessed by number of steps per day. Univariable and multivariable mixed effect models were used to investigate the annual change in STS, 6MWD and number of steps per day.

Results: 202 COPD patients ( $44 \%$ spirometric GOLD stage $1 / 2$, $37 \%$ stage $3,19 \%$ stage 4$)$ with a mean $(\mathrm{min} / \mathrm{max})$ follow-up time of $2.4(0.9 / 6.8)$ years were annually assessed. The number of steps per day decreased significantly over time (annual mean (95\% CI) of $-451.0(-605.3 /-296.6)$ steps, $\mathrm{p}<0.001)$ while STS and 6MWD remained stable (Figure 1).

Conclusion: Our findings suggest that COPD patients are increasingly impaired in their daily PA while exercise capacity remaines stable over time. Thus the longitudinal decline in PA seems not to be explained by a concomitant reduction in exercise tolerance.

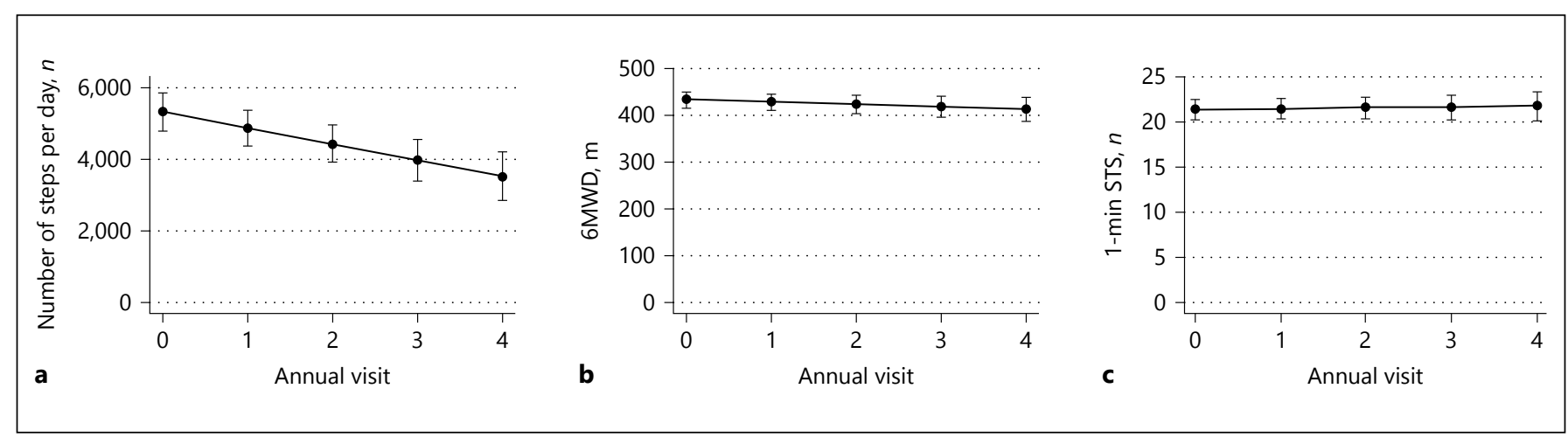

Fig. 1. (for Abstract P55). 


\section{P56 \\ Cardiopulmonary Adaptation in Pediatric Patients with Pectus Excavatum or Pectus Carinatum}

\author{
S. Ramadan ${ }^{1}$, S. Toso ${ }^{2}$, J. Wilde ${ }^{3}$, R. Corbelli ${ }^{1}$, M. Beghetti ${ }^{4}$, \\ C. Barazzone Argiroffo ${ }^{1}$, P. Lascombes ${ }^{5}$, I. Ruchonnet-Métrailler ${ }^{1}$ \\ ${ }^{1}$ Pediatric Pulmonology Unit, ${ }^{2}$ Radiology Unit, ${ }^{3} \mathrm{CURCP}$, \\ ${ }^{4}$ Pediatric Cardiology Unit, ${ }^{5}$ Pediatric Orthopedic Division, \\ Geneva University Hospitals, Geneva, Switzerland
}

Introduction: Pectus excavatum (PE) and Pectus carinatum (PC) are the most frequent morphological chest wall abnormalities with a prevalence between $0.1-2.06 \%$ live births for PE and $0.06 \%$ live births for PC. Even if both conditions differ in their physiopathology, each can affect cardiac and lung function. The objective of this study is to report cardiopulmonary parameters in pediatric patients suffering from these malformations.

Methods: This retrospective study was conducted in the department of pediatrics of the University Hospitals of Geneva, between January 2015 and December 2017. All patients presenting a congenital pectus malformation were eligible. Patients presenting a pectus malformation following a thoracic surgery were excluded. Clinical exam, radiological, pulmonary and cardiac evaluations were performed for each included patients.

Results: 110 patients were eligible. We included 32 patients with $\mathrm{PE}$ and 36 patients with PC who had a complete evaluation.

Among these patients $41 \%$ of $\mathrm{PE}$ and $16.2 \%$ of PC have a positive familial history of chest wall abnormalities. Spirometric evaluations were normal. Cardiac measurements show a normal ventricular ejection fraction in both PE and PC. $42 \%$ of patients with PC and $12.5 \%$ of patients with PE complain of thoracic pain or exercise difficulties independently of their Haller index.

Conclusion: Our preliminary results show that PC and PE have no clear impact on pulmonary or cardiac function. How-

Table 1. Data of patients with PE and PC (for Abstract P56)

\begin{tabular}{lll}
\hline & PE & PC \\
\hline Age (year) & 13 & 14 \\
Sex ratio (M/F) & $30 / 2$ & $34 / 4$ \\
Height (cm) & 163.3 & 170.9 \\
Weight (kg) & 48.7 & 53.9 \\
BMI (kg/m $\left.{ }^{2}\right)$ & 18.30 & 18.5 \\
Haller index & 4.7 & 2.4 \\
FVC (z-score) & $-1.0 .8 \pm 1.33$ & $-0.015 \pm 1.01$ \\
FEV1 (z-score) & $-0.94 \pm 1.51$ & $0.25 \pm 1.3$ \\
FEF 25-75 (z-score) & $-0.33 \pm 1.24$ & $0.19 \pm 0.99$ \\
LVEF (\%) & $60 \%$ & $61 \%$ \\
RVEF (\%) & $54 \%$ & $58 \%$ \\
\hline
\end{tabular}

Results are mean \pm SD; Pectus excavatum $(\mathrm{PC})$; Pectus carlnatum (PC); Forced vital capacity (FVC), Forced expiratory volume in $1 \mathrm{~s}$ (FEV1); Forced expiratory flow (FEF 25-75); Left ventricular election fraction (LVEF); Right ventricular ejection fraction (RVEF). ever a complete lung function with exercise test might help to define more accurately the cardiopulmonary impact in these patients.

\section{P57 \\ Telehealth Care vs. Standard Care in COPD - An International Randomised Controlled Trial (Interim Analysis)}

\author{
F. Rassouli ${ }^{1}$, F. Baty' ${ }^{1}$, D. Stolz ${ }^{2}$, M. Kohler ${ }^{3}$, R. Thurnheer ${ }^{4}$, T. Brack ${ }^{5}$, \\ C. Kähler ${ }^{6}$, M. Tamm ${ }^{2}$, S. Widmer ${ }^{1}$, U. Tschirren ${ }^{1}$, M.H. Brutsche ${ }^{1}$
}

${ }^{1}$ Clinic for Pulmonology and Sleep Medicine, Kantonsspital St.Gallen, St. Gallen, ${ }^{2}$ Clinic for Pulmonology, University Hospital Basel, Basel, ${ }^{3}$ Clinic for Pulmonology, University Hospital Zurich, Zurich, ${ }^{4}$ Clinic for Internal Medicine, Cantonal Hospital Münsterlingen, Münsterlingen, ${ }^{5} \mathrm{Clinic}$ for Internal Medicine, Cantonal Hospital Glarus, Glarus, Switzerland, ${ }^{6} \mathrm{Clinic}$ for

Pulmonology, Waldburg-Zeil-Kliniken, Wangen, Germany

Introduction: Different studies investigating telehealth care (THC) for COPD have shown conflicting results. We published a feasibility trial, which showed excellent patient adherence and satisfaction with our THC approach ${ }^{1}$. Here, we present an interim analysis of the consecutive international randomised-controlled trial.

Methods: Patients are randomly assigned to one group with a crossover after 6 months. Patients in the intervention daily answer 6 questions, focused on the early recognition of exacerbations (AECOPD), using Evita by Swisscom (extended for COPD by the study team $)^{1}$. We daily analyse all entries and react according to a pre-specified action plan. Patients in the control receive standard care without THC. In all, the COPD assessment test (CAT) is measured once weekly. Primary endpoint is HRQOL according to CAT.

Results: Currently, 56 of 175 patients have completed the trial. The overall mean CAT score was 15.3 points. During the intervention, the intraindividual CAT was significantly lower than during the control (mean -0.6 points, $\mathrm{p}=0.001$ ). $23 \%$ of patients improved their CAT more than 2 points, $62 \%$ were stable and $15 \%$ worsened while being in the intervention phase. Satisfaction with COPD care (visual analogue scale) tended to be better after the intervention as compared to the control (mean 8.8 vs. 8.4 points, $\mathrm{p}=$ 0.46). Hospitalisation rate due to AECOPD was 0.18 per patientyear (py) during the intervention vs. 0.25 per py during the control. COPD-related costs were $4619 \mathrm{CHF}$ per py during the intervention vs. $8230 \mathrm{CHF}$ per py during the control.

Conclusion: After completing 32\% of 175 patients, we found a discrete but significant improvement of HRQOL as measured by CAT after 6 months of our THC. Satisfaction with care was already high before the intervention and tended to increase further with THC. We found a lower hospitalisation rate during the intervention and consecutively relevantly lower total COPD-related costs. 


\section{P58}

\section{Right Atrial Pressure at Rest and during Exercise in Patients with and without Pulmonary Hypertension: A Retrospective Analysis}

\section{P. Bader, L. Herren, S. Saxer, M. Lichtblau, C. Berlier, E. Schwarz, M. Furian, K. Bloch, S. Ulrich}

Pulmonary Division, University Hospital of Zurich, Zürich, Switzerland

Introduction: Right atrial pressure (RAP) as measured during right heart catheterisation is of prognostic relevance in patients with pulmonary hypertension $(\mathrm{PH})$. Pulmonary hemodynamics during exercise are of increasing interest in the diagnosis and assessment of prognosis of $\mathrm{PH}$, but little is known about the course of the RAP during exercise.

Methods: Data of 275 patients who underwent supine bicycle exercise right heart catheterization between 10/2008 to $12 / 2015$ were analysed. The course of RAP at rest and during exercise was compared between patients grouped into $\mathrm{PH}$ (mean pulmonary arterial pressure $(\mathrm{mPAP}) \geq 25 \mathrm{~mm} \mathrm{Hg}$ ), exercise-induced $\mathrm{PH}$ (exPH, $\mathrm{mPAP}>30 \mathrm{~mm} \mathrm{Hg}$ and $\mathrm{mPAP} /$ cardiac output $>3 \mathrm{WU}$ ) and no $\mathrm{PH}$.

Results: RAP at rest was significantly higher in $\mathrm{PH}(8.2 \mathrm{~mm} \mathrm{Hg}$ (95\% CI 7.6 to 8.8$)$ ) compared to exPH (5.7 $\mathrm{mm} \mathrm{Hg}$ (4.9 to $6.5, \mathrm{p}<$ 0.001 ) and no-PH $6.5 \mathrm{~mm} \mathrm{Hg}$ (5.7 to $7.3, \mathrm{p}=0.002)$ ). RAP significantly increased at maximal exercise in $\mathrm{PH}$ (by $5.3 \mathrm{~mm} \mathrm{Hg}$ (4.6 to $6.0, \mathrm{p}<0.001$ vs. no-PH) and exPH by $3.7 \mathrm{~mm} \mathrm{Hg}(2.5$ to $4.9, \mathrm{p}=$ 0.027 vs. no $\mathrm{PH})$. RAP at rest was predicted by the mPAP whereas RAP-increase during exercise by age, BMI, group and mPAP increase.

Conclusion: RAP at rest is higher in patients with $\mathrm{PH}$ compared to exPH and no $\mathrm{PH}$ and $\mathrm{mPAP}$ is its independent predictor. Since $\mathrm{PH}$ patients increase RAP during exercise, exercise induced changes in RAP may be used to detect a failing right heart function at an early stage.

\section{P59 \\ Temporal Stability of the Discriminative Power of Exhaled Nitric Oxide (FeNO) before and after Experimental Rhinovirus Challenge}

\author{
A. Sinha ${ }^{1}$, E. Delgado Eckert ${ }^{1}$, X. Binbin ${ }^{2}$, R. Lutter ${ }^{3}$, P.J. Sterk' ${ }^{4}$, \\ U. Frey ${ }^{1}$ \\ ${ }^{1}$ Medicine, University Children's Hospital, Basel, Basel, \\ Switzerland, ${ }^{2}$ Bordeaux Population Health Research Center, \\ Univ. Bordeaux, Inserm, Bordeaux, France, ${ }^{3}$ Department of Lung \\ Immunology, ${ }^{4}$ Medicine Respiratory, Academic Medical Center, \\ University of Amsterdam, Amsterdam, The Netherlands
}

Introduction: Respiratory biomarkers such as fractional exhaled Nitric Oxide (FeNO) are dynamic and adaptively fluctuate over time, especially in asthma (Stern et al., 2011.). Previous reports have also shown that viral infections may elevate levels of
FeNO in breath (Gouw et al., 1998). FeNO being such a fluctuating biomarker, prone to perturbation by viral infections, the question arises as to how good FeNO levels can be for discriminating between healthy and asthmatic subjects.

Hypothesis: FeNO levels discriminate between healthy and asthmatic subjects, despite its temporal fluctuations and susceptibility to perturbation by viral infections.

Aim: To assess the temporal stability of the discriminative power of FeNO before and after an experimental rhinovirus challenge.

Methods: 12 mild asthmatic and 12 healthy adult volunteers have been prospectively followed up for 3 months, thrice weekly. It was a bi-phasic study, first two months (21-visits) constitute the stable phase where the participants are clinically stable. This proceeded a rhinovirus challenge (unstable phase) and follow up for the next 1 month (12-visits). Repeated FeNO assessment (3x weekly) were performed.

Analysis: Mean FeNO levels of healthy subjects at each visit was compared to their corresponding values of asthmatic subjects using Mann-Whitney test.

Results: FeNO levels varied temporally in healthy [mean$14.9925 \mathrm{ppb}, \mathrm{CV}-0.4524$ ] and asthmatic subjects [mean-61.069 ppb, CV-0.8491]. At baseline their group means were significantly different [16 (0.4272) and 59.0833 (0.8635), respectively, $p<$ $0.002]$. FeNO levels in healthy and asthmatic groups remained significantly different $(p<0.05)$ during the stable phase ( 21 visits) and also after the viral challenge unstable phase- 12 visits.

Conclusion: FeNO, despite its fluctuations, shows stable discriminative power to distinguish between healthy and asthmatic subjects even after experimental viral challenge. Determining the robustness of discriminative power of various biomarkers to temporal variability and viral infections under controlled conditions are crucial to determine their clinical usefulness.

\section{P60 \\ Sleep Apnea in Tibetans and Han Long-Term High Altitude Residents}

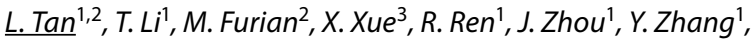
J. He ${ }^{3}$, S. Ulrich' ${ }^{2}$, K.E. Bloch ${ }^{2}$ X. Tang ${ }^{1}$

${ }^{1}$ Sleep Medicine Center, West China Hospital, Sichuan University, Chengdu, China, ${ }^{2}$ Sleep Disorders Center, Dept. of Respiratory Medicine, University Hospital Zurich, Zurich, Switzerland, ${ }^{3}$ Department of Cardiology, Diqing Tibetan Autonomous Prefectural People's Hospital, Shangri-La, China

Introduction: Obstructive sleep apnea (OSA) is associated with adverse health outcomes in lowlanders. The propensity to OSA may be modified by ethnic and environmental influences. We compared the prevalence of sleep apnea between Tibetans and Han long-term residents at high altitude.

Method: 86 consecutive long-term residents ( $>5 \mathrm{y}$ ) of ShangriLa, $3200 \mathrm{~m}$, China, referred for evaluation of snoring or witnessed apnea underwent clinical and questionnaire evaluation and polysomnography (PSG) at the local hospital at $3200 \mathrm{~m}$.

Results: In 42 Tibetans, 38 men, median age $50 \mathrm{y}$, body mass index (BMI) $25.1 \mathrm{~kg} / \mathrm{m}^{2}$, forced expiratory volume in 1s (FEV1) 
93\%pred., the apnea/hypopnea index (AHI) was 55.3/h. In 44 Hans, 32 men, age $47 \mathrm{y}$, BMI $24.4 \mathrm{~kg} / \mathrm{m}^{2}$, FEV1 98\%pred., the AHI was $25.8 / \mathrm{h}(\mathrm{P}<0.001$ vs. Tibetans). In multiple regression analysis controlling for age, sex, BMI, Epworth sleepiness score (ESS) and several other known predictors of OSA, the AHI was positively correlated with Tibetan vs. Han ethnicity (coefficient $+19.8 / \mathrm{h}, 95 \%$ $\mathrm{CI}+8.4$ to $+31.3, \mathrm{P}=0.001)$. Moreover, in 40 men, 20 Tibetans and 20 Hans, matched for age, BMI and ESS, the AHI in Tibetans was higher than in Hans (47.0 vs. 29.4/h, P = 0.028). In 36 men, 18 Tibetans and 18 Hans, matched for age, BMI and AHI, ESS was similar (6.5 vs. $5.5, \mathrm{P}=0.628)$. The mean nocturnal oxygen saturation (SpO2) was lower [median 85\% (quartiles $83 ; 88)$ vs. $89 \%(87 ; 90)$ ] and the apnea duration longer $[22 \sec (20 ; 24)$ vs. $18 \sec (17 ; 20)$ sec] in Tibetan vs. Han ( $\mathrm{P}<0.0001$, both instances).

Conclusions: Our data suggest that long-term residents at high altitude of Tibetan ethnicity have a greater prevalence of sleep apnea than Han Chinese, possibly due to differences in control of ventilation.

\section{P61 \\ Generic Longitudinal and Real-Time Cloud-Based Monitoring for Hospital- and Home-Based Non- Invasive Ventilation - A Proof-of-Concept-Study}

\author{
G.J.V. Benz ${ }^{1}$, B. Schillig' ${ }^{1}$ D. Wattenhofer ${ }^{2}$, F. Michel ${ }^{3}$, M. Brutsche \\ ${ }^{1}$ Pneumologie, Kantonsspital St.Gallen, St. Gallen, ${ }^{2}$ Cyberfish, \\ Biberbrugg, ${ }^{3}$ SGP, Basel, Switzerland
}

Background: Non-invasive ventilation (NIV) is an evidencebased treatment for respiratory failure. Different NIV-modes or ventilators are often applied sequentially. Real-time NIV-monitoring can facilitate adaptation through faster reduction of $\mathrm{CO} 2$ and NIV-tolerance. Not all manufacturers supply real-time-interfaces and graphical interfaces vary greatly.

Aim: To create a generic cloud-based platform to unify the graphical interface provided by the industry and allow real-time monitoring for hospital- and home-based NIV.

Methods: The required software and communication module was developed in collaboration with Cyberfish AG (Biberbrugg, Switzerland). Two NIV ventilator manufacturers cooperated. Löwenstein provided full access to real-time data (Prismavent 40). Limited access to offline data was given by Resmed (Stellar 150).

Results: Separate GSM-modules were created for the two ventilators allowing detailed capture of ventilation data; ventilator settings, real-time pressure curves and web-based post-hoc downloads of NIV-sessions were retrieved on a manufacturer independent user interface.

Conclusion: Real-time data acquisition of NIV sessions is feasible, independent of manufacturer and location. It may facilitate adaptation and management of NIV. Resources might be better aligned with individual needs, e.g. check-up intervals. The platform is likely to improve care for patients in remote areas or shortly after NIV-initiation. In the EU manufacturers are obliged by law to provide unencrypted ventilator interfaces. It is therefore suggested that steps be taken to facilitate further development of a manufacturer-independent platform in Switzerland.

\section{P62}

A Descriptive and Cross-Sectional Multicentric Study of Long-Term Home Non-Invasive Ventilation: Adaptive Servo-Ventilation Population

\section{Cantero $^{1}$, P. Pasquina ${ }^{2}$, D. Adler ${ }^{2}$, A.B. Younossian ${ }^{3}$, M. Prella ${ }^{4}$,} P. Gasche ${ }^{2}$, J.-P. Janssens ${ }^{2}$

${ }^{1}$ Department of General Internal Medicine, ${ }^{2}$ Department of Pulmonology, Geneva University Hospitals, Geneva, ${ }^{3}$ Department of Pulmonology, La Tour Hospital, Meyrin, ${ }^{4}$ Department of Pulmonology, Lausanne University Hospital, Lausanne, Switzerland

Background: Prescription of adaptive-servo-ventilation (ASV) has increased considerably in our area over the last 20 years as a result of technical improvements and ease of use. However, indications for ASV as well as the population treated by ASV are poorly defined.

Aim of study: Describe the indications for which ASV is prescribed, ventilator settings, and compliance.

Methods: Cross-sectional multicentric study of all patients undergoing long term ( $>3$ months) home non-invasive ventilation in Geneva or Vaud (01.06.2016-31.06.2017).

Results: Patients included: 171 patients $(86 \%$ men, median age 71 yrs; IQR 60-77).

Indications for ASV: sleep-related breathing disorders (SRBD, $\mathrm{n}=171)$ : emerging complex sleep apnea syndrome (ECSA) (100 (58.6\%)), central sleep apnea syndrome (CSA) (49 (28.6\%)), obstructive sleep apnea syndrome (OSA) (13 (7.6\%)). 9 (5.2\%) SRBD patients with unknown subgroup.

Origin of CSA: neurologic: 13 (26.6\%); cardiac: $11(22.4 \%)$; drug-related: 8 (16.3\%); idiopathic: $6(12.2 \%)$; cardiac and neurologic: 4 (8.2\%); missing data: 7 (14.3\%).

Among CSA patients, 25 (51\%) had an echocardiography, median LVEF was 60\% (IQR 55-65); 7 (4.1\%) had a LVEF $<45 \%$.

Initiation of ASV: electively: 148 (86.5\%) among whom 107 (62.6\%) after failure of nCPAP; after an acute episode of respiratory failure: $6(3.5 \%)$. Started in a hospital ward for $129(75.4 \%)$, as outpatient for $16(9.3 \%)$. Medical follow-up: pulmonologist in private practice: 34 (19.9\%); hospital center: 110 (64.3\%); both: 27 (15.8\%). Data from ventilator software: mean compliance $6.2 / \mathrm{h}$ (IQR 4.4-7.4), mean AHI 1/h (IQR 0-4).

Conclusion: Patients under ASV are a heterogeneous mostly male comorbid and aged population, with mainly ECSA and CSA. Most ASV are initiated electively, in a hospital ward by a hospital center although pulmonologists in private practice play a substantial role. Among CSA patients, $4 \%$ had a LVEF $<45 \%$ and $40 \%$ had no echocardiography, in spite of the publication of SERVE-HF in 2014. 
Table 1. Baseline characteristics (for Abstract P62)

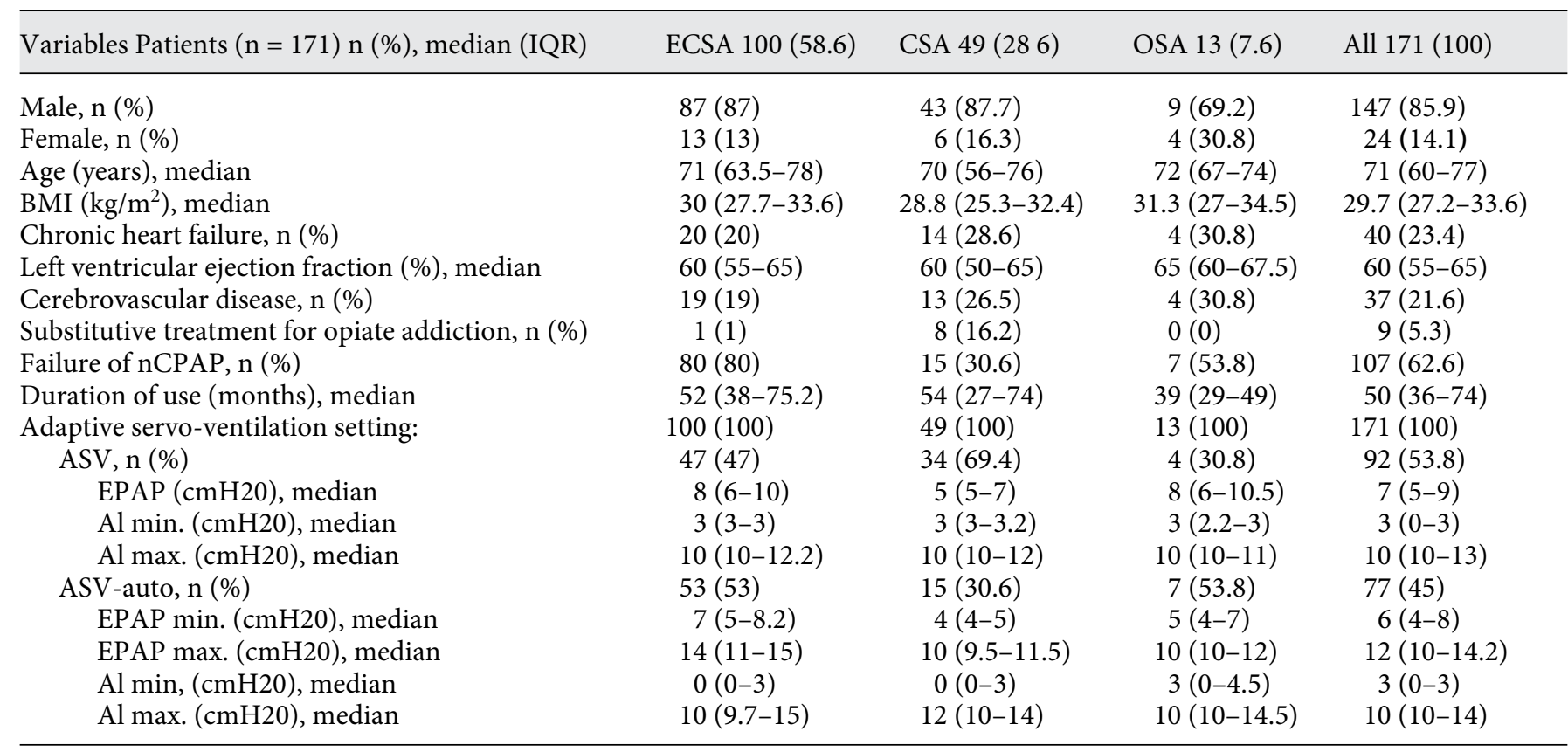

ECSA (emerging complex sleep apnea syndrome), CSA (central sleep apnea syndrome), OSA (obstructive sleep apnea syndrome); 9 (5.2\%) SRBD patients with unknown subgroup.

\section{P63 \\ Gait Speed, Hospital Readmission and Death Following Acute Hypercapnic Respiratory Failure}

$\underline{\text { D. Adler }}{ }^{1}$, E. Dupuis-Lozeron ${ }^{2}$, P.M. Soccal' ${ }^{1}$ J.-P. Janssens ${ }^{1}$

${ }^{1}$ Service de Pneumologie, ${ }^{2}$ Service d'Épidémiologie Clinique, HUG, Genève, Switzerland

Background: Long term outcome of patients admitted to the hospital for acute hypercapnic respiratory failure (AHRF) is poor. Simple tools to stratify patients for risk of readmission and death may help clinicians to individualize care in AHRF survivors.

Aim: To prospectively evaluate the ability of gait speed, a marker of frailty, to predict future readmission and death in patients admitted to the ICU for AHRF.

Methods: 78 patients who survived AHRF treated by NIV in the ICU were recruited prospectively. Gait speed and all predictive variables were measured 15 days after resolution of acute respiratory failure and before discharge to home or to longer term facility. Patients confined to the bed were given an arbitrary gait speed value of $0 \mathrm{~m} / \mathrm{sec}$. COPD was diagnosed with spirometry. Heart failure was diagnosed with clinical rules and echocardiography.

Results: Baseline characteristics of the cohort: median age 69 IQR (63-75); 53\% men; median FEV1 $50 \%$ of predicted IQR (39-58). 10/78 (13\%) patients had an imputed gait speed of $0 \mathrm{~m} /$ sec. Mean gait speed was $0.68 \mathrm{~m} \pm 0.45 \mathrm{~m} / \mathrm{sec}$. Higher gait speed at hospital discharge was associated with decreased risk of read- mission or death at 6 months (OR: $0.27,95 \%$ CI: $0.08-0.81, \mathrm{p}=$ 0.025 ) and decreased one year mortality (OR: $0.22,95 \%$ CI: $0.04-0.97, \mathrm{p}=0.059)$. In a multivariate model also incorporating age, BMI, $\mathrm{FEV}_{1}$ and heart failure, only gait speed remained associated with all-cause readmission at 6 months (OR: 0.20, 95\% CI: $0.05-0.75, \mathrm{p}=0.022$ ). Gait speed (OR: 0.08, 95\% CI: $0.01-$ $0.73, \mathrm{p}=0.042$ ) in addition to FEV1 (OR: 0.93, 95\% CI: $0.85-$ $0.99, \mathrm{p}=0.04)$ and heart failure (OR: 11.4, $95 \mathrm{CI}: 1.56-151, \mathrm{p}=$ 0.032 ) were associated with 1 year mortality when adjusted for age and BMI. Slow gait speed was associated with higher risk of

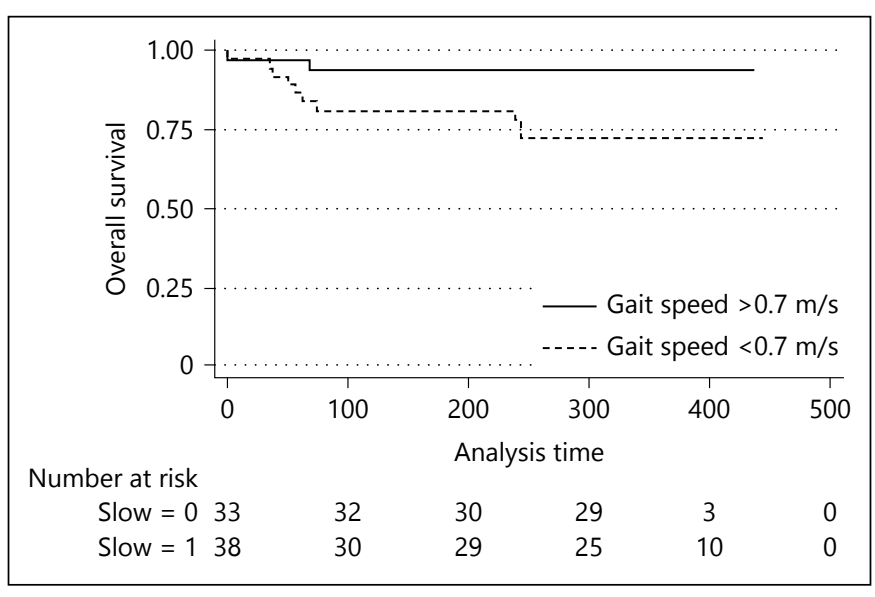

Fig. 1. (for Abstract P63). 
mortality within the 1 year observation period, $\mathrm{p}=0.021$ (Figure $1)$.

Conclusion: Gait speed independently predicts the risk of readmission and death in patients surviving AHRF.

\section{P64}

\section{Association of Pulmonary Hypertension with Sleep Apnea in Highlanders}

\author{
T.M. Sooronbaev' ${ }^{1}$, M. Furian ${ }^{2}$, M. Mademilov' ${ }^{1}$, B. Estebesova', \\ B. Osmonov' ${ }^{1}$, N. Marajapov', U. Sheraliev' 1 , P.M. Scheiwiller ${ }^{2}$, \\ M. Lichtblau' ${ }^{2}$, S. Saxer ${ }^{2}$, P. Bader ${ }^{2}$, T. Rochat ${ }^{3}$, S. Ulrich ${ }^{2}$, K.E. Bloch ${ }^{2}$
}

${ }^{1}$ National Center for Cardiology and Internal Medicine, Bishkek, Kyrgyzstan, ${ }^{2}$ Pulmonary Division and Sleep Disorders Center, University Hospital of Zurich, Zurich, ${ }^{3}$ Pulmonary Division, University Hospital Geneva, Geneva, Switzerland

Background: In a recent cross-sectional study in Kyrgyz highlanders we found an association of obstructive sleep apnea (OSA) with high altitude pulmonary hypertension (HAPH), a chronic altitude related illness causing exercise limitation and impaired quality of life (Latshang Eur Respir J 2017). A similar association in lowlanders is uncommon. The current study further evaluates a potential role of OSA in promoting HAPH.

Methods: Data from an ongoing, prospective, open cohort study in residents of the Aksay highlands (Kyrgyzstan, altitude 3-4000 m) were analyzed. Study participants in 2017 and in at least one previous examination were included. Clinical assessments, sleep studies and echocardiography deriving mean pulmonary artery pressure (mPAP) from pulmonary artery flow acceleration were performed yearly.

Results: In 71 highlanders (36 females) 268 yearly examinations were performed over a mean (SD) observation period of $4.7 \pm$ $0.6 \mathrm{y}$. Baseline age was $44 \pm 12 \mathrm{y}$, mPAP $28 \pm 8 \mathrm{~mm} \mathrm{Hg}$, apnea/ hypopnea index (AHI) $18.2 \pm 17.0 / \mathrm{h}$, mean nocturnal oxygen saturation $88 \pm 4 \%$ and body mass index (BMI) $26.9 \pm 4.7 \mathrm{~kg} / \mathrm{m}^{2}$. In univariate linear regression analysis $\mathrm{mPAP}$ increased with age $(0.35 \mathrm{~mm} \mathrm{Hg} /$ year, $\mathrm{p}<0.001)$. In multivariate analysis, AHI $(0.05$ $\mathrm{mm} \mathrm{Hg} /$ event $/ \mathrm{h}, \mathrm{p}=0.013$ ) was independently associated with mPAP controlling for age $(0.31 \mathrm{~mm} \mathrm{Hg} /$ year, $\mathrm{p}<0.001)$, and BMI $\left(0.23 \mathrm{~mm} \mathrm{Hg} / \mathrm{kg} / \mathrm{m}^{2}, \mathrm{p}=0.042\right) \mathrm{R}^{2}=0.41$.

Conclusions: Our prospective observation over several years confirms an association of sleep apnea with MPAP in highlanders further supporting a role of sleep apnea in development of $\mathrm{HAPH}$.

Grant Support: OPO foundation, Lunge Zürich.

\section{P65 \\ Bridging Host-Bacteria-Virus Interactions to Clinical Outcome in Lung Transplantation}

E. Bernasconi ${ }^{1}$, C. Pattaroni ${ }^{1,2}$, D.-A. Wurlod ${ }^{1}$, J.-D. Aubert ${ }^{1}$, A. Koutsokera ${ }^{1}$, B.J. Marsland ${ }^{1,2}$, L.P. Nicod ${ }^{1}$

'Service de Pneumologie, CHUV and STCS, Lausanne, Switzerland, ${ }^{2}$ Immunology Alfred Hospital, Monash University, Melbourne, VIC, Australia

Introduction: Long-term lung transplant stability is limited by the onset of obstructive and/or restrictive pathophysiological processes, which irreversibly progress towards distinct types of chronic rejection that lack predictive factors. The healthy lung is believed to be tied to carriage of a balanced microbiota that includes bacteria and viruses. We hypothesized that bioinformatics integration of lung microbiota and host gene expression features during routine follow-up of lung transplant recipients may help improve patient risk stratification.

Methods: DNA and RNA were isolated from 135 bronchoalveolar lavages (BAL) obtained from 36 patients up to 24 months post-transplantation. Bacterial community composition was determined using $16 \mathrm{~S}$ sequencing. Anellovirus-specific load and host gene expression for a set of 31 targets involved in host-microbe interactions were quantified using qPCR. We derived a bioinformatics tool based on Similarity Network Fusion (SNF) for i) creating a comprehensive network that integrates shared and complementary information from lung microbiota composition and host gene expression datasets, and ii) BAL sample clustering.

Results: SNF analysis identified four sample clusters, between which transplant recipients were commonly observed to switch over time. In addition to stand out in the combined analysis of bacterial community composition and host gene expression, two clusters linked to highly active host humoral and cellular immunity, respectively, displayed low bacterial and anellovirus carriage, BAL neutrophils and infection rate. In sharp contrast, a high-inflammatory cluster that markedly differed based on this full set of criteria was linked to a significantly lower ratio of current FEV1 to best post-transplant FEV1, and a corresponding higher rate of current obstructive syndrome.

Conclusion: Integrating the dynamic and interrelated features of lung bacteria, viruses and host cells informs on local conditions, which align with host immunity and organ function, and has potential for predicting those lung transplant recipients with a trajectory towards obstructive pathophysiology. 


\section{P66}

\section{Systemic Levels of Hyaluronidase-1 Are Increased in COPD - Implication in COPD Exacerbations}

\begin{abstract}
E. Papakonstantinou' ${ }^{1}$, M. Roth ${ }^{2}$, M. Tamm ${ }^{1}$, L. Boeck' ${ }^{1}$ A. Scherr ${ }^{1}$, J. Rakic ${ }^{1}$, R. Louis ${ }^{3}$, B. Milenkovic ${ }^{4}$, W. Boersma ${ }^{5}$, K. Kostikas ${ }^{6}$, F. Blasi ${ }^{7}$, J. Aerts ${ }^{8}$, G. Rohde ${ }^{9}$, A. Lacoma ${ }^{10}$, A. Torres ${ }^{11}$, T. Welte ${ }^{12}$, D. Stolz ${ }^{1}$

${ }^{1}$ Pneumology, ${ }^{2}$ Department of Biomedicine, University of Basel, Basel, Switzerland, ${ }^{3}$ Pneumology, University of Liege, CHU Liege, Belgium, ${ }^{4}$ Clinic for Pulmonary Diseases, University of Belgrade, Belgrade, Serbia, ${ }^{5}$ Department of Pneumology, Medisch Centrum Alkmaar, Alkmaar, The Netherlands, ${ }^{6}$ Department of Pneumology, University of Thessaly, Larissa, Greece, ${ }^{7}$ Department of Pathophysiology and Transplantation, Università degli Studi di Milano, Milan, Italy, ${ }^{8}$ Department of Pneumology, Amphia Hospital/Erasmus MC, Breda, ${ }^{9}$ Department of Respiratory Medicine, Maastricht University Medical Center, Maastricht, The Netherlands, ${ }^{10}$ Department of Microbiology, Hospital Universitari Germans Trias i Pujol, CIBER Enfermedades Respiratorias, Badalona, ${ }^{11}$ Department of Pneumology, Hospital Clinic, Barcelona, Barcelona, Spain, ${ }^{12}$ Department of Pneumology, Medizinische Hochschule Hannover, Hannover, Germany
\end{abstract}

Introduction: Hyaluronic acid (HA) constitutes the major glycosaminoglycan in lung tissue, with HA degradation products, produced by hyaluronidases (HYAL), being implicated in several lung diseases including COPD. We have previously shown that acute exacerbations of COPD (AECOPD) are associated with increased levels and activity of HYAL in bronchoalveolar lavage (BAL).

Methods: In the present study, we measured circulating levels of HYAL-1 in 638 patients with COPD, GOLD II-IV, >10PY, included in the PROMISE cohort, at stable state, at exacerbation and four weeks after exacerbations, using ELISA.

Results: Systemic levels of HYAL-1 were positively correlated with age $(\mathrm{p}<0.001)$ and comorbidities $(\mathrm{p}<0.001)$ and negatively correlated with 6-minute walking distance $(\mathrm{p}=0.001)$. HYAL-1 was significantly increased in COPD as compared to sex- and agematched controls $(\mathrm{p}<0.001)$. Furthermore, HYAL-1 was increase at moderate $(\mathrm{p}=0.004)$ and severe exacerbations $(\mathrm{p}=0.003)$ and remained significantly higher 4 weeks after exacerbations $(\mathrm{p}<$ 0.001).

Conclusion: Our results indicate that COPD is associated with increased systemic levels of HYAL-1 and this may lead to pro-inflammatory degradation of HA in the lung. Furthermore, the increased levels of HYAL- 1 at exacerbations may contribute to a persistent airway inflammation and subsequent lung function decline.

\section{P67 \\ Hepatocyte Growth Factor Increases and Activates Lung Stem Cells in the Bleomycin Injured Lung}

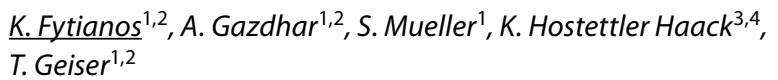

${ }^{1}$ Department of Bio-Medical Research, University of Bern, Bern, ${ }^{2}$ Respiratory Medicine, Bern University Hospital, Bern, ${ }^{3}$ Pneumology, University Hospital Basel, Basel, ${ }^{4}$ Department of Biomedicine, University of Basel, Bael, Switzerland

Introduction: Idiopathic Pulmonary Fibrosis (IPF) is a fatal, age-related disease, with complicated pathophysiology. Mesenchymal Stem Cells (MSC) in IPF lungs have anti-fibrotic properties. Hepatocyte Growth Factor (HGF) plays key role in lung repair and regeneration, however its effect on lung MSCs is unknown. We hypothesize that HGF overexpression activates quiescent lung MSCs and support lung repair.

Methods: Adult male rats were instilled with bleomycin intratracheally. After 7 days, electroporation mediated gene transfer was performed with either HGF or empty vectors ( $n=5$ per group). 7 days after gene transfer, the rats were sacrificed and lung, Broncho Alveolar Lavage (BAL) and blood were collected. Singe cell homogenate from fresh lungs, BAL, Bone Marrow (BM) and blood were analyzed by flow cytometry for MSC characterization using defined rat MSC markers (CD29, CD44, CD90 and CD105) and markers for cell cycle analysis. Additionally, collagen content in the lung was measured and histological analysis was performed.

Results: Previously, our group has shown that HGF gene transfer reduced fibrosis. Hydroxyproline content was reduced after HGF gene transfer $(230 \pm 36.94 \mu \mathrm{g} / \mathrm{mg}$ vs $393.2 \pm 139.2 \mu \mathrm{g} / \mathrm{mg}$ compared to control). Sirius Red and Hematoxylin-Eosin (H\&E) staining further confirmed reduced collagen in histology sections. Lung MSCs were considerably increased after HGF gene transfer (2507 \pm 175 MSCs vs $270 \pm 132$ MSCs, per 30,000 recorded single cells from lung homogenates) $(\mathrm{p}<0.05)$. However, MSCs in BAL and blood did not show any significant differences. Moreover, lung MSC cell cycle analysis showed an almost 3-fold increase of MSCs in the lung $(27.5 \pm 3.2 \%$ of activated MSCs compared to $10.7 \pm 7.5 \%$ in the control group).

Conclusions: Our results indicate that HGF gene transfer increases MSC cell numbers in the fibrotic lung and in particular enhances the amount of activated MSCs, therefore possibly supporting amelioration of lung fibrosis. 


\section{P68}

\section{Lung Resident Stem/Progenitor Cells Express Markers of Pluripotency}

P. Khan ${ }^{1}$, A. Gazdhar ${ }^{2}$, L. Fang ${ }^{1}$, M. Roth ${ }^{1}$, M. Tamm 1 , T. Geiser ${ }^{2}$, $\underline{\text { K. Hostettler }}^{1}$

${ }^{1}$ Clinics of Respiratory Medicine and Department of Biomedicine, University Hospital Basel, Basel, ${ }^{2}$ Division of Pulmonary Medicine, University Hospital Bern, Bern, Switzerland

Introduction: Stem/progenitor cells exist in the lungs of patients with fibrotic lung diseases and have been successfully cultured ex vivo. We have previously shown that cultured lung resident stem/progenitor cells exhibited mesenchymal differentiation potential (adipocytes, osteoblasts, myoblasts, chondroblasts) and, interestingly, also differentiate into epithelial cells. In this study we further investigated the characteristics and differentiation potential of lung-derived stem/progenitor cells.

Methods: Stem/progenitor cells were isolated from lung tissue obtained from patients with fibrotic lung diseases. To study differentiation capacity, the cells were cultured in embryoid body me- dium for 3 weeks and then stained for $\alpha$-fetoprotein, $\alpha$-smooth muscle actin, and $\beta$-tubulin. Expression of pluripotency markers (Oct4, Nanog, Sox2, Tra-1-60, SSEA4) was detected by immunofluorescence (IF) and/or TaqMan PCR. Alkaline phosphatase expression was determined in a co-culture of stem/progenitor cells with human lung fibroblasts.

Results: Lung resident stem/progenitor cells, cultured in embryoid body medium for three weeks, stained positive for $\alpha$-fetoprotein (early endoderm marker), $\alpha$-smooth muscle actin (mesoderm marker), and $\beta$-tubulin (ectoderm marker). Furthermore, stem/progenitor cells stained positive for the pluripotency markers Oct 4, Nanog, SOX2, Tra-1-60 and SSEA4 and expressed Oct 4, Nanog and SOX2 RNA. In addition, lung stem/progenitor cells express much higher levels of alkaline phosphatase, when compared to human lung fibroblasts.

Conclusion: Or data provide evidence that lung-derived stem/ progenitor cells may be pluripotent: (1) lung stem/progenitor cells seem to differentiate into cells that form tissue of the three primordial germ layers of the embryo, (2) the cells express a number of pluripotency markers, (3) cells express high levels of alkaline phosphatase. In future studies it will be important to investigate the functional role and possible therapeutic use of these lung resident stem/progenitor cells in fibrotic lung diseases. 


\section{Author Index}

\section{Respircition}

Adler, D. P34, P39, P62, P63

Aerts, J. P66

Aigner, F. P09

Allali, G. P39

Amirav, I. P07

Arnet, I. P16

Asthma-COPD-Overlap

Syndrome diversification study P42

Aubert, J.-D. P12, P65

Bader, P. P58, P64

Barazzone Argiroffo, C. P56

Barben, J. P07

Baty, F. P09, P46, P51, P52, P57

Becker, A. P33

Bédat, B. P50

Beghetti, M. P56

Beishekeeva, G. P45

Benz, G.J.V. P61

Berlier, C. P18, P58

Bernasconi, E. P65

Berra, G. P04, P39

Bieber, T. P15

Bierreth, F. P26

BILD Study Group P01

Binbin, X. P59

Blasi, F. P66

Bloch, K. P58

Bloch, K.E. P45, P60, P64

Boeck, L. P17, P66

Boersma, W. P66

Bonvin, P. P28

Boon, M. P02

Bopp, M. P48

Borie, R. P53

Boss, A. P33

Brack, T. P55, P57

Braun, S. P23

Briault, A. P53

Brutsche, M. P09, P46, P51, P52, P55, P61

Brutsche, M.H. P42, P57

Bumm, R. P21, P27

Burney, P.G.J. P45

Cantero, C. P34, P62

Capova, G. P20

Casaulta, C. P02, P07

Clarenbach, C. P55

Corbelli, R. P56

Cordier, J.-F. P53
Cottin, V. P53

Crowley, S. P02

Daccord, C. P29, P53

De Hogh, K. P48

Delgado Eckert, E. P59

Diamanti, E. P29

Diedrich, J. P14

Dieterle, T. P05, P06, P14, P16, P26

Diethelm, P. P08

Dressel, H. P48

Duchna, H.-W. P15, P20

Dupuis-Lozeron, E. P39, P63

Dürr, S. P16

Duvoisin, G. P22

Estebesova, B. P45, P64

Fang, L. P37, P38, P68

Faouzi, M. P53

Filippi, S. P30

Fink, G. P03

Fitting, J.-W. P47

Flamm, A.-L. P16

Fouzas, S. P01

Franchetti, L. P41

Frey, M. P55

Frey, U. P01, P59

Furian, M. P45, P58, P60, P64

Furrer, M. P21, P27, P32, P35

Fytianos, K. P67

Ganassi, K. P19

Gasche, P. P62

Gazdhar, A. P67, P68

Geiser, T. P67, P68

Geraplangsub, S. P25

Gondouin, A. P53

Gonzales, C. P13

Gonzalez, M. P11

Goutaki, M. P02, P07

Gregoriano, C. P16

Grize, L. P17

Guerin, S. P22

Hachulla, A.-L. P24

Haile, S.H. P33

Halbeisen, F. P02, P07

Halter, J. P54

Hässig, G. P32

He, J. P60

Herren, L. P58
Hersberger, K.E. P16

Hess, T. P23

Hirsch, H. P17, P54

Hofer, M. P23

Hogg, C. P02, P07

Hostettler, K. P68

Hostettler Haack, K. P67

Hoyler, K. P19

Huss-Mischler, K. P32

Irani, S. P55

Jacobshagen, M. P28

Jahn, K. P41, P54

Jandus, P. P28

Janssens, J.-P. P04, P34, P62, P63

Jaramillo Vogel, D. P44

Jendricke, T. P05, P06

Junker, L. P54

Kaelin, R.M. P08

Kaenpon, T. P31

Kähler, C. P57

Karadag, B. P02

Karenovics, W. P50

Kern, L. P42, P46, P52

Khan, P. P68

Khouatra, C. P53

Klein, P. P10

Koerner-Rettberg, C. P02

Kohler, M. P33, P55, P57

Koob, M. P22

Kostikas, K. P49, P66

Koutsokera, A. P65

Kozu, K. P46, P52

Kraemer, R. P42

Kraus, L. P15

Kubena, P. P20

Kuehni, C. P02, P07

Kuhn, B.K. P44

Lacoma, A. P66

Lador, F. P24

Lascombes, P. P56

Latshang, T. P32

Latshang, T.D. P21, P27

Latzin, P. P01

Lawi, D. P39

Lazor, R. P29, P53

Ledergerber, M. P03

Lenoir, A. P12, P47

Lettau, F. P33
Leuenberger, M. P42

Leuppi, J. P05, P06, P55

Leuppi, J.D. P14, P16, P26

Li, T. P60

Lichtblau, M. P18, P58, P64

Long, O. P43

Longatti, A. P28

Louis, R. P17, P66

Lovis, A. P43

Lucas, J. P02, P07

Ludwig, P. P21, P27

Lutter, R. P59

Mademilov, M. P64

Maier, S. P05, P06, P16

Marajapov, N. P64

Marmy, J.L. P14

Marques-Vidal, P.M. P47

Marsland, B.J. P65

Martin, M. P07

Maurer, E. P02

Maxén, J. P21

Mazurek, H. P02

Menz, G. P15, P20

Merkel, A. P36

Michel, F. P36, P61

Michelitsch, C. P35

Milenkovic, B. P66

Moya, E. P07

Mueller, S. P67

Naduvilekoot, A. P05, P06, P26

Napairee, C. P25

Nicod, L. P11, P43

Nicod, L.P. P47, P65

Nielsen, K. P07

Noble, S. P24

Noirez, L. P43

Nydegger, A. P22

Ogal, M. P10

Ogna, A. P11, P43

Oriol, M. P11

Osmonov, B. P64

Özçelik, U. P02

Papakonstantinou, E. P17, P66

Pasquina, P. P34, P62

Pattaroni, C. P65

Perez Valdes, C.R. P12

Piquilloud, L. P43 
Plojoux, J. P04

Prella, M. P34, P62

Proietti, E. P01

Puhan, M. P48

Rakic, J. P17, P66

Ramadan, S. P56

Rassouli, F. P57

Rausch, J. P44

Regamey, N. P19

Rejmer, P. P33

Ren, R. P60

Ritz, N. P03

Rochat, I. P22

Rochat, T. P64

Rohde, G. P66

Röösli, M. P48

Rossi, C. P33

Roth, M. P37, P38, P66, P68

Rothe, T. P27

Ruchonnet-Métrailler, I. P56

Sadowski, S.M. P50

Saenghirunvattana, P. P13

Saenghirunvattana, S. P13, P25, P31

Santamaria, F. P02

Savic, S. P54

Saxer, S. P18, P58, P64

Scheiwiller, P.M. P64
Scherr, A. P66

Schillig, B. P61

Schilter, D. P17

Schindler, C. P17

Schlegel-Wagner, C. P19

Schneider, T. P46, P52

Schoch, O.D. P51

Schoop, R. P10

Schulzke, S. P01

Schumann, D. P40, P41, P49, P54

Schwarz, E. P18, P58

Schwarz, E.I. P33

Schwendener, K. P19

Schwerk, N. P02

Sheraliev, U. P64

Siangproh, C. P13

Siebeneichler, A. P40

Sievi, N. P33, P55

Simon, J. P43

Sinha, A. P59

Skaventzos, I. P29

Soccal, P.M. P04, P34, P63

Somaini, G. P27, P35

Sooronbaev, T.M. P45, P64

Sterk, P.J. P59

Stöberl, A. P33

Stocker, M. P19

Stolz, D. P17, P37, P38, P40, $\mathrm{P} 41, \mathrm{P} 49, \mathrm{P} 54, \mathrm{P} 57, \mathrm{P} 66$
Stradling, J.R. P33

Strassmann, A. P48

Strobel, W. P54

Studer, L. P40

Sturzenegger, R. P35

Suter, A. P01

Suthisri, K. P13

Swiss National Cohort Study Group P48

Szavay, P. P19

Tamm, M. P17, P36, P37, P38, P40, P41, P49, P54, P57, P66, P68

Tan, L. P60

Tang, X. P60

Teres, C. P24

Thesenvitz, E. P05, P06

Thiel, S. P33

Thouvenin, G. P07

Thurnheer, R. P55, P57

Toktogulova, N. P45

Tonko, S. P51

Torres, A. P66

Toso, S. P56

Triponez, F. P50

Tschirren, U. P57

Turk, A. P48

Udry, M. P11
Ulrich, S. P18, P45, P58, P60, P64

Usemann, J. P01, P03

Vanek, P. P35

Vollenweider, P. P47

Vremaroiu, P. P43

Vrugt, B. P44

Wang, C. P48

Wani, J. P23

Wattenhofer, D. P61

Welte, T. P66

West, E. P48

Wetchanien, P. P25

Widmer, S. P57

Wilde, J. P56

Wurlod, D.-A. P65

Xue, X. P60

Yiallouros, P. P02

Younossian, A.B. P34, P62

Zannin, E. P01

Zhang, Y. P60

Zhou, J. P60

Züsli, S. P05, P06 